Marlos Mangini

\title{
MÉTODO DOS ELEMENTOS FINITOS GENERALIZADOS PARA ANÁLISE DE ESTRUTURAS EM CASCA DE REVOLUÇÃO
}

Dissertação apresentada à Escola de Engenharia de São Carlos da Universidade de São Paulo, como parte dos requisitos para a obtenção do Título de Mestre em Engenharia de Estruturas.

Orientador: Sergio Persival Baroncini Proença

\author{
SÃO CARLOS
}

Dezembro de 2006 



\section{Dedicatória}

Ao grande amigo Robson Calixto dos Reis,

pois, sem a sua amizade e ajuda este trabalho sequer teria sido iniciado. 


\section{Agradecimentos}

$\grave{\mathrm{A}}$ Deus.

Aos meus pais, Antonio Carlos Mangini e Maria Sezerino Mangini por tudo que fizeram por mim e pelas oportunidades que me propiciaram e aos meus irmãos Krys Mangini e Karla Mangini pela força e incentivo.

Aos tios Evilásio Badziak e Terezinha Sezerino Badziak que por diversas vezes estiveram ao meu lado prestando auxilio.

À todos os amigos de Curitiba, que mesmo eu estando distante, não esqueceram da nossa amizade.

Aos professores da Universidade Federal do Paraná pelo auxilio em chegar até aqui.

Ao professor Sergio Persival Baroncini Proença pela atenção concedida, dedicação em auxiliar-me compreendendo minhas falhas e por toda orientação no decorrer da pesquisa.

Aos professores do departamento Luiz Eduardo e André Beck pelas dicas de programação e "macetes" do Fortran. Aos amigos Walter e Luiz Aquino com suas estratégias e programas para driblar as dificuldades de montagem do trabalho e a Oscar Alfredo Garcia de Suarez pelas contribuições e ajuda no desenvolvimento desta dissertação.

À todos os funcionários do Departamento de Engenharia de Estruturas que contribuíram direta ou indiretamente para o desenvolvimento do trabalho e a CAPES pelo apoio financeiro.

Aos amigos com quem tive a oportunidade de conviver durante o período dessa pós-graduação no departamento, Alice, Ricardo, Tatiane, Elian, Marta, Tatiana, Fernanda Calmon, Rodolfo, Ceará (Pedro), Rogério, Sudano, Caio, Paccola, em especial a Rafaelle Tiboni e Michell Macedo Alves pela grande ajuda quando cheguei a São Carlos.

Aos membros da gloriosa Sala 5, Mineiro (Edson), Eduardo, Codá, Edmar e Rodrigo pelo companheirismo, auxilio, troca de experiências e pela paciência em aguentarme enquanto escutava as transmissões de futebol pela internet. 
Aos caçulas do departamento de estruturas, Fernanda Madrona, Karla, Camila, Wanderson, Jesus, Raimundo, Vinicius, Jonatas, Antonio, Rafael, João, Pedro Cesar e Rômulo, Rene e Rodrigo Couto que mesmo com um tempo menor de convívio mostram ser pessoas muito legais e ótimas companhias.

Durante essa temporada de SET, conheci muitas pessoas e todas tiveram sua importância, pois foi com elas que dividi os meus dias em São Carlos, valeu mesmo gente, a amizade de vocês foi imprescindível. Aos grandes amigos Ronaldo, Glaucia, Filipe, Thiago Catoia, Saulo, André Dória, Denis, o grande companheiro das "Seções Corujão" no departamento e a Iara que muito me ajudou em momentos difíceis. A todos vocês meu muito obrigado pelos ótimos momentos aqui vividos.

De forma muito especial, gostaria de lembrar aqui de quatro pessoas que sempre estiveram ao meu lado, Luiz Vieira meu grande amigo, Gustavo Siqueira uma das primeiras amizades em São Carlos e a duas meninas por quem tenho um carinho especial e enorme estima, Karenina e Livia. A vocês gostaria de deixar meus mais sinceros agradecimentos, pois sem esse convívio certamente essa temporada sancarlense não teria sido a mesma e sim muito mais difícil e sem graça. 


\section{Resumo}

MANGINI, M. (2006). Método dos Elementos Finitos Generalizados para análise de estruturas em casca de revolução. Dissertação (Mestrado) - Escola de Engenharia de São Carlos, Universidade de São Paulo, São Carlos, 2006.

O presente trabalho está inserido no campo de estudo das cascas axissimétricas, tendo como objetivo a análise de seu comportamento estrutural mediante o desenvolvimento e aplicação de uma ferramenta numérica baseada no Método dos Elementos Finitos Generalizados. A utilização desse recurso é uma alternativa eficaz e difere do Método dos Elementos Finitos convencional pela possibilidade de enriquecimento nodal das funções de aproximação. Como resultado pode-se dispensar o uso de redes muito refinadas. Com o intuito de evidenciar as vantagens do método adotado são apresentados exemplos comparando-se as soluções numéricas obtidas com soluções analíticas ou numéricas geradas com o do Método dos Elementos Finitos convencional. Os resultados obtidos com um pequeno número de elementos finitos e com enriquecimento por funções polinomiais, mostraram-se convergentes já nos primeiros graus de enriquecimento. Desenvolve-se uma análise complementar de convergência baseada em estimativa de erro, mostrando que a metodologia adotada pode proporcionar melhores taxas de convergência em relação ao refino h quando predomina a regularidade da solução. A mesma análise aponta que a combinação dos refinos h e p pode levar a resultados mais precisos, com elevadas taxas de convergência, quando a solução (particularmente suas derivadas) apresentar regularidade menor.

Palavras-chave: cascas axissimétricas, método dos elementos finitos generalizados, enriquecimento, erro de aproximação, convergência. 


\begin{abstract}
MANGINI, M. (2006). Generalized Finite Element Method to analysis of structures in revolution shell. M.Sc Dissertation - São Carlos School of Engineering, University of São Paulo, São Carlos, 2006.
\end{abstract}

The present dissertation is inserted in the field of study of the axisymmetric shells. The objective is to analyze the structural behavior by means of the development and application of a numerical tool based on the Generalized Finite Element Method. The use of this resource is an efficient alternative to the conventional Finite Element Method for the possibility of nodal enrichment of the approach functions. Therefore one can avoid the use of very fine nets. In addition, in order to evidence the advantages of the adopted method, there are shown examples comparing the numerical solutions with analytical or numerical values generated with the conventional Finite Element Method. The results obtained with a small number of elements, including enrichment by polynomial functions, had revealed convergence in the first degrees of enrichment. It is developed a complementary convergence analysis based on estimate of error, showing that the adopted methodology can provide better convergence ratios in relation to the h-refinement, in the cases where the regularity of the solution predominates. The same analysis shows that the combination of the refinement in its versions $\mathrm{h}$ and $\mathrm{p}$ can give more accurate results, by increasing convergence, when the solution (particularly its derivatives) presents lower regularity.

Key-words: axisymmetric shells, generalized finite element method, enrichment, error of approach, convergence. 


\section{Lista de Figuras}

Figura 1 Estrutura espacial e simplificação plana $\quad \ldots \ldots \ldots \ldots \ldots \ldots \ldots \ldots \ldots$

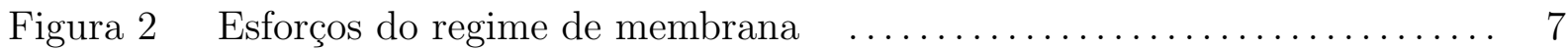

Figura 3 Exemplo de casca de revolução em regime de membrana $\quad \ldots \ldots \ldots \ldots . \quad 8$

Figura 4 Esforços no elemento infinitesimal de membrana $\ldots \ldots \ldots \ldots \ldots \ldots \ldots$

Figura 5 Esforços nos paralelos e meridianos da membrana $\quad \ldots \ldots \ldots \ldots \ldots \ldots \ldots$

Figura 6 Equilíbrio das forças segundo a direção vertical $\quad \ldots \ldots \ldots \ldots \ldots \ldots \ldots \ldots$

Figura 7 Deslocamentos no regime de membrana $\quad \ldots \ldots \ldots \ldots \ldots \ldots \ldots \ldots \ldots \ldots$

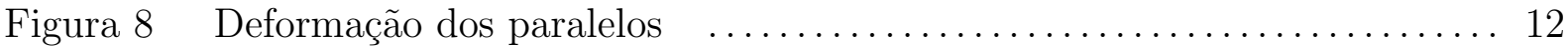

Figura 9 Rotação da tangente ao meridiano $\quad \ldots \ldots \ldots \ldots \ldots \ldots \ldots \ldots \ldots \ldots \ldots \ldots \ldots \ldots \ldots \ldots$

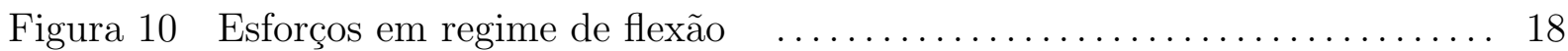

Figura 11 Esforços no elemento infinitesimal em regime de flexão $\ldots \ldots \ldots \ldots \ldots . . .18$

Figura 12 Esforços nos paralelos e meridianos no regime de flexão $\quad \ldots \ldots \ldots \ldots \ldots 19$

Figura 13 Variação da curvatura tangente ao meridiano $\ldots \ldots \ldots \ldots \ldots \ldots \ldots \ldots .21$

Figura 14 Variação da curvatura na direção normal ao meridiano $\ldots \ldots \ldots \ldots \ldots . .22$

Figura 15 Comparação entre cascas e viga sobre base elástica $\quad \ldots \ldots \ldots \ldots \ldots \ldots .25$

Figura 16 Base ortonormal associada a um nó do elemento $\ldots \ldots \ldots \ldots \ldots \ldots \ldots$

Figura 17 Domínio Paramétrico e Vetor posição $\ldots \ldots \ldots \ldots \ldots \ldots \ldots \ldots \ldots \ldots \ldots \ldots$

Figura 18 Bases co-variante e ortonormal para um ponto qualquer do elemento .. 32

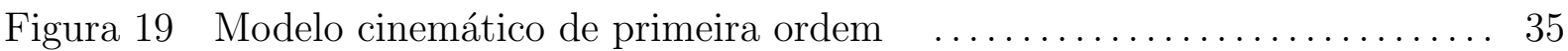

Figura 20 Estado de tensões para o problema axissimétrico $\quad \ldots \ldots \ldots \ldots \ldots \ldots \ldots . \ldots$

Figura 21 Esquema estático para modelagem do problema $\quad \ldots \ldots \ldots \ldots \ldots \ldots \ldots \ldots$

Figura 22 Sub-domínios $\omega_{i}$ e funções de forma $N_{i}(\eta) \quad \ldots \ldots \ldots \ldots \ldots \ldots \ldots \ldots$

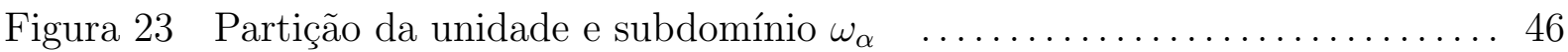

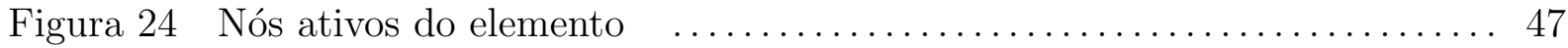


Figura 25 Sistema de referência associado à nuvem $\alpha \quad \ldots \ldots \ldots \ldots \ldots \ldots \ldots \ldots$

Figura 26 Partição da unidade e funções enriquecidas $\quad \ldots \ldots \ldots \ldots \ldots \ldots \ldots \ldots . \ldots$

Figura 27 Parametrização do sistema de coordenadas $\quad \ldots \ldots \ldots \ldots \ldots \ldots \ldots \ldots \ldots$

Figura 28 Funções de aproximação enriquecidas $\quad \ldots \ldots \ldots \ldots \ldots \ldots \ldots \ldots \ldots \ldots \ldots \ldots \ldots \ldots \ldots \ldots \ldots$

Figura 29 Partição da unidade e derivada primeira - polinômio do primeiro grau 56

Figura 30 Partição da unidade e derivada primeira - polinômio do terceiro grau 56

Figura 31 Partição da unidade e derivada primeira - polinômio do quinto grau . 57

Figura 32 Fluxograma básico do programa desenvolvido $\ldots \ldots \ldots \ldots \ldots \ldots \ldots \ldots 6$

Figura 33 Exemplo 01 - Corte esquemático da estrutura $\ldots \ldots \ldots \ldots \ldots \ldots \ldots \ldots$

Figura 34 Momentos fletores $M_{\theta}$ - Solução de referência e refino p $\ldots \ldots \ldots \ldots 66$

Figura 35 Momentos fletores $M_{\varphi}$ - Solução de referência e refino p $\ldots \ldots \ldots \ldots 67$

Figura 36 Forças cortantes $V_{\varphi}$ - Solução de referência e refino $\mathrm{p} \quad \ldots \ldots \ldots \ldots \ldots 6$

Figura 37 Deslocamentos $w$ - Solução de referência e refino p $\ldots \ldots \ldots \ldots \ldots \ldots 67$

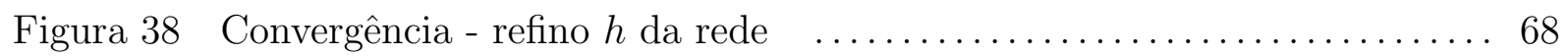

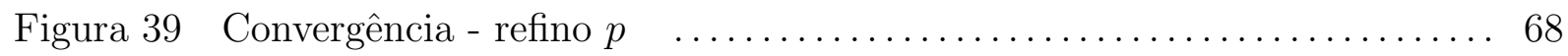

Figura 40 Travamento - Valores normalizados para o deslocamento transversal $\quad \ldots \quad 70$

Figura 41 Exemplo 02 - Corte esquemático da estrutura $\ldots \ldots \ldots \ldots \ldots \ldots \ldots \ldots$

Figura 42 Momentos fletores $M_{\theta}$ - Solução de referência e refino p $\ldots \ldots \ldots \ldots .71$

Figura 43 Momentos fletores $M_{\varphi}$ - Solução de referência e refino p $\quad \ldots \ldots \ldots \ldots .72$

Figura 44 Forças normais $N_{\theta}$ - Solução de referência e refino p $\quad \ldots \ldots \ldots \ldots \ldots \ldots$

Figura 45 Forças normais $N_{\varphi}$ - Solução de referência e refino p $\quad \ldots \ldots \ldots \ldots \ldots .72$

Figura 46 Forças cortantes $V_{\varphi}$ - Solução de referência e refino $\mathrm{p} \quad \ldots \ldots \ldots \ldots \ldots$

Figura 47 Deslocamentos $v$ - Solução de referência e refino p $\ldots \ldots \ldots \ldots \ldots \ldots$

Figura 48 Deslocamentos $w$ - Solução de referência e refino p $\quad \ldots \ldots \ldots \ldots \ldots \ldots$

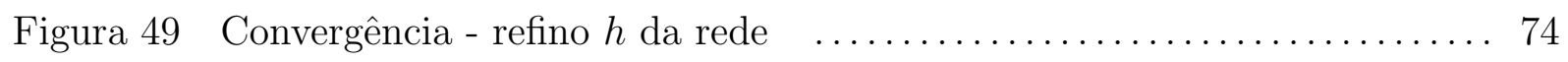

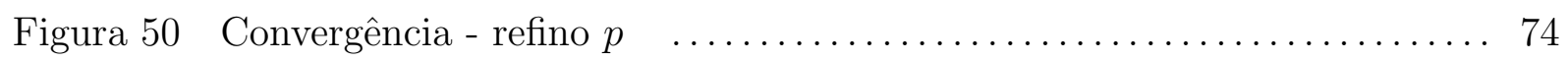

Figura 51 Exemplo 03 - Corte esquemático da estrutura $\ldots \ldots \ldots \ldots \ldots \ldots \ldots \ldots$ 
Figura 52 Momentos fletores $M_{\theta}$ - Solução de referência e refino p $\ldots \ldots \ldots \ldots .76$

Figura 53 Momentos fletores $M_{\varphi}$ - Solução de referência e refino p $\ldots \ldots \ldots \ldots .76$

Figura 54 Forças normais $N_{\theta}$ - Solução de referência e refino p $\ldots \ldots \ldots \ldots \ldots \ldots$

Figura 55 Forças normais $N_{\varphi}$ - Solução de referência e refino p $\ldots \ldots \ldots \ldots \ldots 77$

Figura 56 Forças cortantes $V_{\varphi}$ - Solução de referência e refino p $\quad \ldots \ldots \ldots \ldots \ldots 77$

Figura 57 Deslocamentos $v$ - Solução de referência e refino p $\quad \ldots \ldots \ldots \ldots \ldots \ldots . \ldots 77$

Figura 58 Deslocamentos $w$ - Solução de referência e refino p $\quad \ldots \ldots \ldots \ldots \ldots \ldots \ldots$

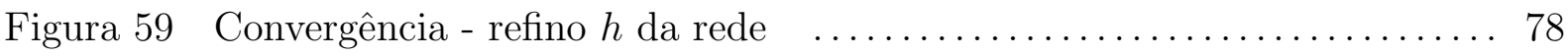

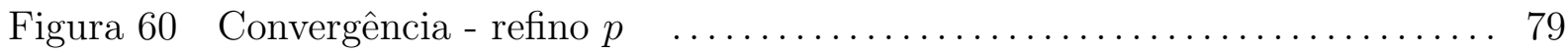

Figura 61 Exemplo 04 - Corte esquemático da estrutura $\ldots \ldots \ldots \ldots \ldots \ldots \ldots \ldots$

Figura 62 Momentos fletores $M_{\theta}$ - Solução de referência e refino p $\ldots \ldots \ldots \ldots \ldots 81$

Figura 63 Momentos fletores $M_{\varphi}$ - Solução de referência e refino p $\ldots \ldots \ldots \ldots .81$

Figura 64 Forças normais $N_{\theta}$ - Solução de referência e refino p $\quad \ldots \ldots \ldots \ldots \ldots . \ldots 1$

Figura 65 Forças normais $N_{\varphi}$ - Solução de referência e refino p $\ldots \ldots \ldots \ldots \ldots . . . . .2$

Figura 66 Forças cortantes $V_{\varphi}$ - Solução de referência e refino p $\ldots \ldots \ldots \ldots \ldots . \quad 82$

Figura 67 Deslocamentos $v$ - Solução de referência e refino p $\ldots \ldots \ldots \ldots \ldots \ldots \ldots 2$

Figura 68 Deslocamentos $w$ - Solução de referência e refino p $\ldots \ldots \ldots \ldots \ldots \ldots \ldots . . . \ldots 3$

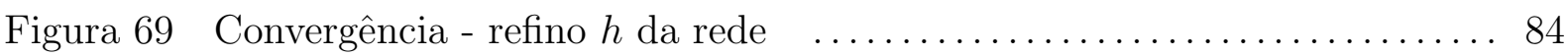

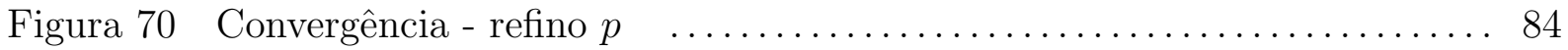

Figura 71 Exemplo 05 - Corte esquemático da estrutura $\ldots \ldots \ldots \ldots \ldots \ldots \ldots \ldots$

Figura 72 Momentos fletores $M_{\theta}$ - Solução de referência e refino p - Placa $\ldots . .86$

Figura 73 Momentos fletores $M_{\theta}$ - Solução de referência e refino p - Tronco de cone 86

Figura 74 Momentos fletores $M_{\varphi}$ - Solução de referência e refino p - Placa $\ldots . . .87$

Figura 75 Momentos fletores $M_{\varphi}$ - Solução de referência e refino p - Tronco de cone 87

Figura 76 Forças normais $N_{\theta}$ - Solução de referência e refino p - Placa $\quad \ldots \ldots \ldots .87$

Figura 77 Forças normais $N_{\theta}$ - Solução de referência e refino p - Tronco de cone 88

Figura 78 Forças normais $N_{\varphi}$ - Solução de referência e refino p - Placa $\ldots \ldots \ldots .88$ 
Figura 79 Forças normais $N_{\varphi}$ - Solução de referência e refino p - Tronco de cone 88

Figura 80 Forças Cortantes $V_{\varphi}$ - Solução de referência e refino p - Placa $\ldots . \ldots . \quad 89$

Figura 81 Forças cortantes $V_{\varphi}$ - Solução de referência e refino p - Tronco de cone 89

Figura 82 Deslocamentos $v$ - Solução de referência e refino p - Placa $\ldots \ldots \ldots$.... 89

Figura 83 Deslocamentos $v$ - Solução de referência e refino p - Tronco de cone $\quad \ldots 90$

Figura 84 Deslocamentos $w$ - Solução de referência e refino p - Placa $\ldots . . . . . .90$

Figura 85 Deslocamentos $w$ - Solução de referência e refino p - Tronco de cone $\quad$. 90

Figura 86 Convergência - refino $h$ da rede $\ldots \ldots \ldots \ldots \ldots \ldots \ldots \ldots \ldots \ldots \ldots \ldots \ldots \ldots \ldots \ldots \ldots \ldots$

Figura 87 Convergência - refino $p \quad \ldots \ldots \ldots \ldots \ldots \ldots \ldots \ldots \ldots \ldots \ldots \ldots \ldots \ldots \ldots \ldots \ldots \ldots \ldots \ldots \ldots \ldots \ldots$

Figura 88 Exemplo 06 - Corte esquemático da estrutura $\ldots \ldots \ldots \ldots \ldots \ldots \ldots \ldots 2$

Figura 89 Momentos fletores $M_{\theta}$ - Solução de referência e refino p $\ldots \ldots \ldots \ldots .93$

Figura 90 Momentos fletores $M_{\varphi}$ - Solução de referência e refino p $\ldots \ldots \ldots \ldots .93$

Figura 91 Forças normais $N_{\theta}$ - Solução de referência e refino p $\ldots \ldots \ldots \ldots \ldots . \ldots 3$

Figura 92 Forças normais $N_{\varphi}$ - Solução de referência e refino p $\ldots \ldots \ldots \ldots \ldots . . . \ldots 4$

Figura 93 Forças cortantes $V_{\varphi}$ - Solução de referência e refino p $\ldots \ldots \ldots \ldots \ldots . . \ldots 4$

Figura 94 Deslocamentos $v$ - Solução de referência e refino p $\ldots \ldots \ldots \ldots \ldots \ldots . . \ldots 4$

Figura 95 Deslocamentos $w$ - Solução de referência e refino p . . ............. 95

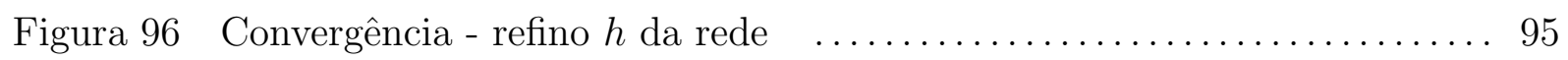

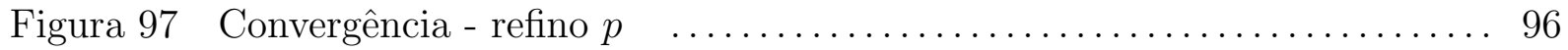




\section{Lista de Tabelas}

Tabela 1 Exemplo 01 - Dados de entrada do modelo $\ldots \ldots \ldots \ldots \ldots \ldots \ldots \ldots . \ldots 5$

Tabela 2 Exemplo 02 - Dados de entrada do modelo $\quad \ldots \ldots \ldots \ldots \ldots \ldots \ldots \ldots \ldots$

Tabela 3 Exemplo 03 - Dados de entrada do modelo $\quad \ldots \ldots \ldots \ldots \ldots \ldots \ldots \ldots \ldots$

Tabela 4 Exemplo 04 - Dados de entrada do modelo $\ldots \ldots \ldots \ldots \ldots \ldots \ldots \ldots$........... 80

Tabela 5 Exemplo 05 - Dados de entrada do modelo $\quad \ldots \ldots \ldots \ldots \ldots \ldots \ldots \ldots$. 85

Tabela 6 Exemplo 06 - Dados de entrada do modelo $\ldots \ldots \ldots \ldots \ldots \ldots \ldots \ldots \ldots 2$ 


\section{Lista de Siglas}

CAPES Coordenação de Aperfeiçoamento de Pessoal de Nível Superior

MEF Método dos Elementos Finitos

MEFG Método dos Elementos Finitos Generalizados

PU Partição da unidade 


\section{Lista de Símbolos}

$\vec{a}_{1}$ a $\vec{a}_{3} \quad$ Vetores componentes da base covariante

$B_{t} \quad$ Matriz das deformações

C Constante dependente da condição de contorno do problema

C Matriz constitutiva

$C_{1}$ a $C_{4} \quad$ Constantes relativas as condições de contorno para resolução o sistema homogêneo

$C(s) \quad$ Função de mapeamento - sistema paramétrico global para o físico global

$C_{1}(\eta) \quad$ Função de mapeamento - sistema local para o paramétrico global

$C_{\nu, E} \quad$ Constante da relação constitutiva

ds Diferencial de comprimento de arco

$d v \quad$ Diferencial do deslocamento - direção da tangente ao meridiano

$d w \quad$ Diferencial do deslocamento - direção normal da tangente ao meridiano

$d \theta \quad$ Diferencial do ângulo $\theta$ - direção dos paralelos

$d \varphi \quad$ Diferencial do ângulo $\varphi$ - direção dos meridiano

$d L_{i} \quad$ Gradiente do espaço das funções de enriquecimento

$d M_{\varphi} \quad$ Diferencial de momento fletor em torno do paralelo

$d N_{\varphi} \quad$ Diferencial de esforço normal na direção tangente ao meridiano

$d Q_{\varphi} \quad$ Diferencial de esforço cortante na direção normal da tangente ao meridiano

D Rigidez a flexão de estruturas de superfície

$e_{i} \quad$ Erro local referente ao nó $i$

$\|e\| \quad$ Norma energia do erro

E Módulo de elasticidade longitudinal do material

$\tilde{E}_{i} \quad$ Valor da solução aproximada

$E_{i}^{r e f} \quad$ Valor da solução de referência

xix 
$f_{1}$ a $f_{3} \quad$ Funções lagrangianas

F $\quad$ Vetor de cargas da estrutura

$\left[F_{\zeta}\right] \quad$ Base covariante

$F_{\alpha}^{p} \quad$ Conjunto das funções de aproximação enriquecidas

$g_{i}$ e $g_{s} \quad$ Funções lagrangianas

J Operador jacobiano

$h \quad$ Espessura da parede da casca

$h_{\alpha} \quad$ Raio da nuvem

H Operador de para obtenção o tensor das deformações

$\vec{i}_{\theta} \quad$ Versor do eixo $\theta$

$\vec{i}_{r}(\theta) \quad$ Versor do eixo $r$

$J \quad$ Matriz jacobiana - transformação entre os sistemas de coordenadas

$K \quad$ Matriz de rigidez da estrutura

$L(\ldots) \quad$ Operador

$L_{i, \alpha} \quad$ Conjunto formado por monômios de uma base polinomial

$M_{\varphi} \quad$ Momento fletor entorno dos paralelos

$M_{\theta} \quad$ Momento fletor entorno dos meridianos

$n \quad$ Número de nós da rede

$\vec{n} \quad$ Vetor normal a um ponto do elemento

$N_{i} \quad$ Funções de forma

N Matriz cinemática do problema

$N_{\text {nuv }} \quad$ Número de nuvens da rede de elementos finitos

$N_{\varphi} \quad$ Esforço normal na direção tangente ao meridiano

$N_{\theta} \quad$ Esforço normal na direção tangente ao paralelo

$N_{\omega} \quad$ Número de nuvens que cobrem o domínio

$Q_{\varphi} \quad$ Esforço cortante na direção normal da tangente ao meridiano

$\left[Q_{X}\right] \quad$ Base ortonormal associada a um ponto do elemento 
$\left[Q_{k}\right] \quad$ Matriz de rotação

$O \quad$ Origem do sistema de coordenadas

O Centro de curvatura do elemento infinitesimal na direção dos paralelos

$O_{1} \quad$ Centro de curvatura do elemento infinitesimal na direção dos meridianos

p Ordem polinomial

$p_{\varphi} \quad$ Carregamento externo na direção da tangente ao meridiano

$p_{\rho} \quad$ Carregamento externo na direção perpendicular a tangente ao meridiano

$\vec{p} \quad$ Carregamento externo

P Resultante das forças externas na direção do eixo de revolução da casca

$r \quad$ Direção radial - sistema global

$r \quad$ Raio de curvatura no plano do meridiano

$r_{0} \quad$ Raio de curvatura no plano do paralelo

$r_{0}^{\prime} \quad$ Raio de curvatura na direção do paralelo após a deformação

$R$ e $R_{c} \quad$ Raio de curvatura da casca esférica

R Operador de transformação de coordenadas

$s \quad$ Coordenada paramétrica global

t $\quad$ Espessura da casca

$t_{k} \quad$ Espessura do elemento em $\mathrm{k}$

$\vec{t} \quad$ Vetor tangente a um ponto do elemento

$\vec{u} \quad$ Vetor de deslocamentos

$\vec{u}_{k} \quad$ Vetor de deslocamentos associado ao nó k do elemento

$\vec{u}_{o k} \quad$ Vetor de deslocamentos sobre a superfície média associado ao nó k do elemento

$\bar{u} \quad$ Deslocamento prescrito

U Vetor dos deslocamentos

$\bar{U} \quad$ Vetor dos deslocamentos prescritos

$\vec{U}_{g} \quad$ Vetor de deslocamentos referido ao sistema global

$\vec{U}_{l} \quad$ Vetor de deslocamentos referido ao sistema local 
Deslocamento - direção da tangente ao meridiano

$\vec{v}_{2 k}$ e $\vec{v}_{3 k} \quad$ Versores componentes da base ortonormal associada ao nó k

$v_{2 k}^{r}$ e $v_{2 k}^{z} \quad$ Componentes de $\vec{v}_{2 k}$ segundo as direções de $\vec{i}_{r}(\theta)$ e $\vec{k}$

$v_{3 k}^{r}$ e $v_{3 k}^{z} \quad$ Componentes de $\vec{v}_{3 k}$ segundo as direções de $\vec{i}_{r}(\theta)$ e $\vec{k}$

$\vec{v}_{2 k G}(\theta) \quad$ Vetor referido à base global

$\vec{v}_{2 k L}(\theta) \quad$ Vetor referido à base local

$\vec{V}_{3 k} \quad$ Vertor associado a espessura do elemento em $\mathrm{k}$

$V_{\alpha}^{p} \quad$ Espaço das funções de enriquecimento

$w \quad$ Deslocamento - direção normal da tangente ao meridiano

W $\quad$ Peso associado ao ponto de integração

$x \quad$ Posição de um ponto no domínio

$x_{\alpha} \quad$ Posição de uma nuvem do domínio

$\bar{x} \quad$ Posição de um ponto no domínio da nuvem

$\vec{X}_{k} \quad$ Vetor posição associado do elemento

$\vec{X}_{k}^{s} \quad$ Vetor posição associado à extremidade superior do elemento: nó $\mathrm{k}$

$\vec{X}_{k}^{i} \quad$ Vetor posição associado à extremidade inferior do elemento: nó k

$\vec{X}_{k, \text { med }} \quad$ Vetor posição associado ao nó k do elemento

$z \quad$ Direção vertical - sistema global

$\alpha \quad$ Angulo de abertura da casca

$\alpha \quad$ Rotação da tangente ao meridiano

$\alpha \quad$ Nó ativo ou nuvem

$\gamma \quad$ Angulo auxiliar

$\gamma \quad$ Coeficiente utilizado na formulação das cascas

$\partial \Omega_{N} \quad$ Contorno carregado

$\partial \Omega_{D} \quad$ Contorno com deslocamento prescrito

$\Omega \quad$ Domínio do problema 
$\triangle A B_{n} \quad$ Variação no comprimento do arco devido ao deslocamento segundo a normal

$\triangle A B_{t} \quad$ Variação no comprimento do arco devido ao deslocamento segundo a tangente

$\Delta x \quad$ Área de influencia do nó para cálculo da norma energia do erro

$\varepsilon \quad$ Tensor das deformação

$\varepsilon_{t} \quad$ Tensor das deformação referido a base ortonormal local

$\varepsilon_{\varphi} \quad$ Deformação na direção da tangente ao meridiano

$\varepsilon_{\theta} \quad$ Deformação na direção dos paralelos

$\varepsilon_{n n}$ e $\varepsilon_{t t} \quad$ Deformações segundo as direções normal e tangente

$\zeta \quad$ Coordenada paramétrica

$\nu \quad$ Coeficiente de Poisson

$\varphi \quad$ Ângulo medido no plano do meridiano da casca

$\varphi_{k} \quad$ Giro sofrido pela normal devido ao efeito do cisalhamento

$\phi \quad$ Função ponderadora

$\phi_{\alpha} \quad$ Funções de forma

$\rho \quad$ Direção normal à tangente ao meridiano

$\sigma_{t t} \quad$ Tensão normal

$\sigma_{n n} \quad$ Tensão normal nula

$\tau_{n t} \quad$ Tensão cisalhante

$\tau_{n \theta}$ e $\tau_{\theta t} \quad$ Tensões cisalhantes nulas

$\sigma_{\varphi} \quad$ Tensão normal segundo a direção da tangente ao meridiano

$\sigma_{\theta} \quad$ Tensão normal segundo a direção da tangente ao paralelo

$\xi \quad$ Deslocamento horizontal - sistema global

$\xi \quad$ Abscissa normalizada - sistema referenciado à nuvem

$\eta \quad$ Deslocamento vertical - sistema global

$\eta \quad$ Coordenada paramétrica

$\theta \quad$ Angulo medido sobre o plano do paralelo da casca

$\theta \quad$ Coordenada paramétrica 
$\chi_{\varphi} \quad$ Mudança de curvatura do meridiano

$\chi_{\theta} \quad$ Mudança de curvatura do paralelo

$\psi_{i}(\eta) \quad$ Funções de aproximação enriquecidas

$\omega_{\alpha} \quad$ Subdomínio

$\Omega_{e} \quad$ Domínio paramétrico do elemento

$\sum \quad$ Domínio paramétrico global

$\sum F_{V} \quad$ Somatório das forças segundo a direção do eixo vertical

$\sum F_{\rho} \quad$ Somatório das forças segundo a direção perpendicular à tangente ao meridiano

$\sum M_{\theta} \quad$ Somatório dos momentos fletores em torno dos meridianos

$\nabla \quad$ Gradiente

$\nabla_{r} \vec{u}(r, z) \quad$ Gradiente dos deslocamentos referido ao sistema global de coordenadas

$\nabla_{r} \vec{u}(\eta, \zeta)$ Gradiente dos deslocamentos referido ao sistema local de coordenadas

$\nabla_{t} \vec{u}(\eta, \zeta) \quad$ Gradiente dos deslocamentos referido à base ortonormal local 


\section{Sumário}

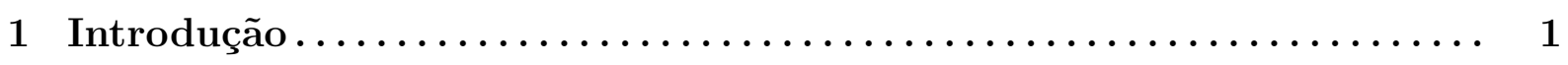

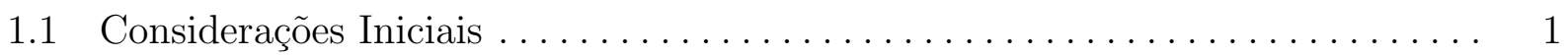

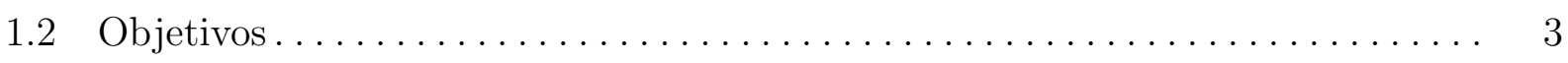

1.3 Justificativas . . . . . . . . . . . . . . . . . . $\ldots \ldots \ldots$

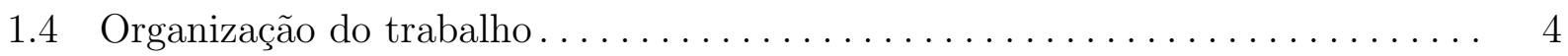

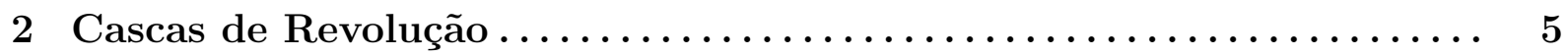

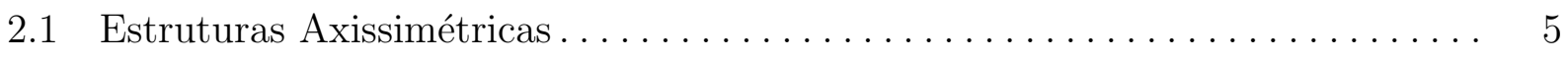

2.2 Hipóteses normalmente usadas em abordagens analíticas simplificadas . . . . . 5

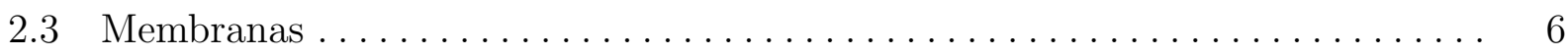

2.4 Equacionamento do regime de membrana $\ldots \ldots \ldots \ldots \ldots \ldots \ldots \ldots \ldots$

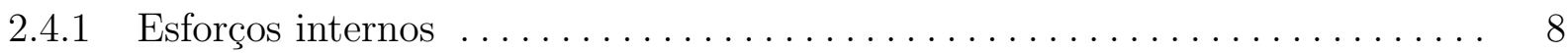

2.4.2 Deslocamentos no regime de membrana $\ldots \ldots \ldots \ldots \ldots \ldots \ldots \ldots \ldots \ldots$

2.4.3 Rotação da tangente ao meridiano no regime de membrana . . . . . . . . . 15

2.5 Cascas em regime de flexão $\ldots \ldots \ldots \ldots \ldots \ldots \ldots \ldots \ldots \ldots \ldots \ldots \ldots \ldots \ldots \ldots \ldots$

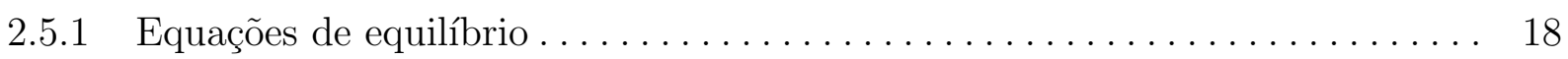

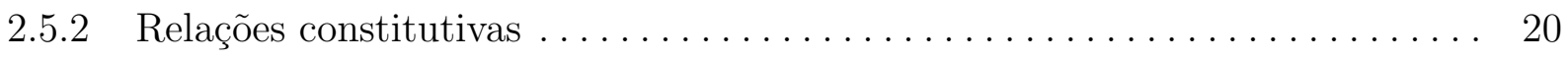

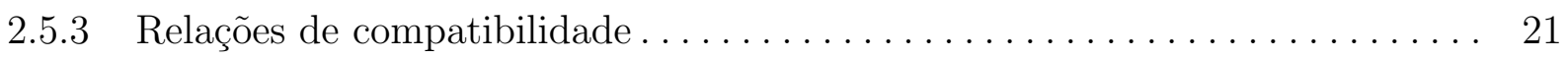

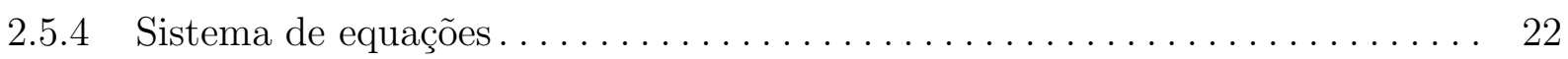

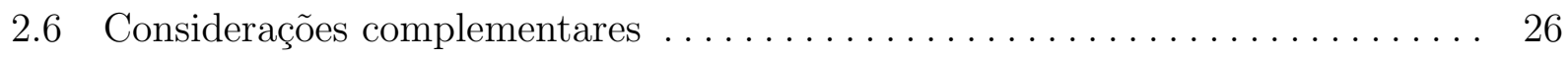

3 Elemento Finito Axissimétrico $\ldots \ldots \ldots \ldots \ldots \ldots \ldots \ldots \ldots \ldots \ldots \ldots \ldots \ldots \ldots \ldots$

3.1 Características geométricas do elemento $\ldots \ldots \ldots \ldots \ldots \ldots \ldots \ldots \ldots \ldots$ 
3.1.1 Base ortonormal associada a um nó do elemento $\ldots \ldots \ldots \ldots \ldots \ldots \ldots$

3.1 .2 Mapeamento na definição da geometria do elemento . . . . . . . . . . . . 29

3.1.3 Base co-variante e ortonormal para um ponto qualquer do elemento $\ldots \ldots \ldots 31$

3.2 Modelo cinemático de Reissner-Mindlin . . . . . . . . . . . . . . . . . . . 34

3.2.1 Modelo de Reissner-Mindlin aplicado ao caso axissimétrico . . . . . . . . . 34

3.3 Equações constitutivas - Problema axissimétrico $\ldots \ldots \ldots \ldots \ldots \ldots \ldots \ldots \ldots$

3.4 Forma fraca do problema - Método de Galerkin . . . . . . . . . . . . . . . 38

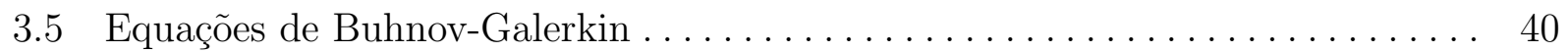

3.5.1 Deformações - Sistema local de coordenadas . . . . . . . . . . . . . . . . . . 41

3.5.2 Matriz de Rigidez e Vetor de Forças nodais equivalentes . . . . . . . . . . . . . 44

4 Método dos Elementos Finitos Generalizados.................. 45

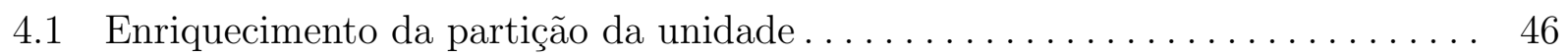

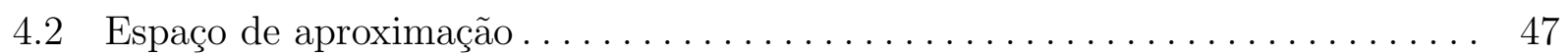

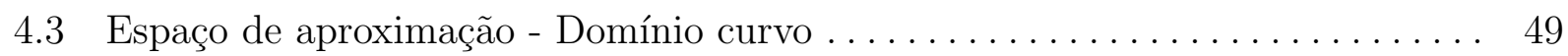

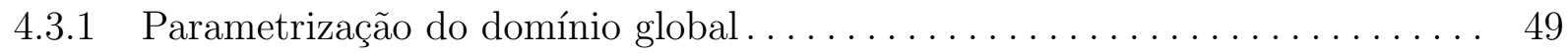

4.3.2 Deslocamentos com o uso do espaço de aproximação enriquecido . . . . . . . . 53

4.4 Construção da matriz de rigidez e vetor de forças nodais equivalentes - Base

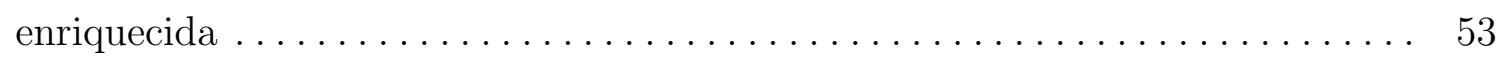

5 Programa Desenvolvido .......................... 55

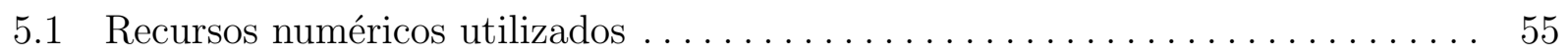

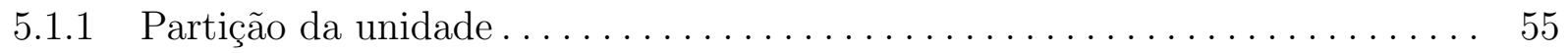

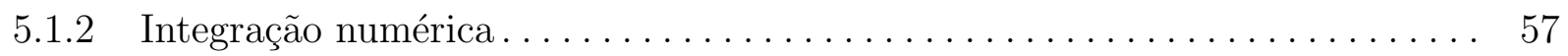

5.1 .3 Rotina para resolução do sistema $-K \cdot \vec{U}=\vec{F} \ldots \ldots \ldots \ldots \ldots \ldots \ldots$

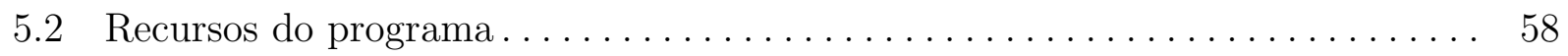

5.2 .1 Etapas de pré-processamento e pós-processamento . . . . . . . . . . . 59

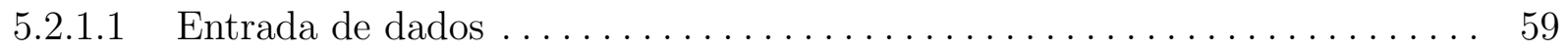




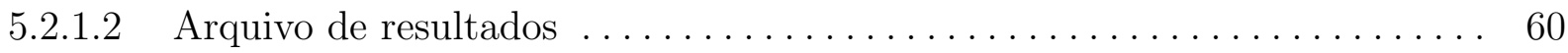

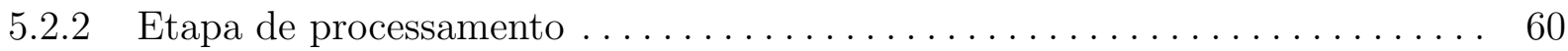

5.2.2.1 Cálculo dos deslocamentos e esforços internos . . . . . . . . . . . . . 60

5.2 .3 Tipos de elementos implementados $\ldots \ldots \ldots \ldots \ldots \ldots \ldots \ldots \ldots \ldots \ldots \ldots$

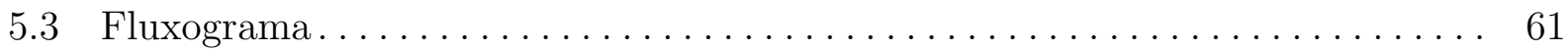

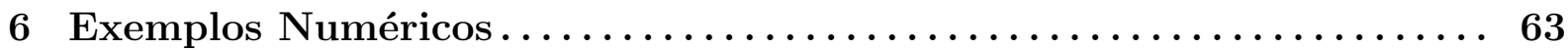

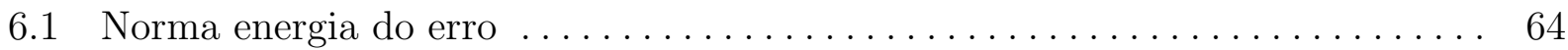

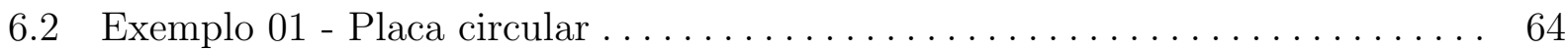

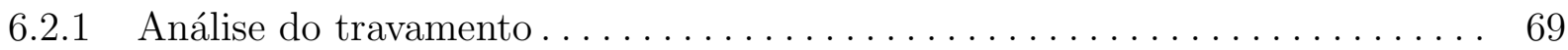

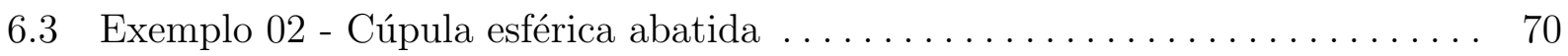

6.4 Exemplo 03 - Cilindro sob pressão $\ldots \ldots \ldots \ldots \ldots \ldots \ldots \ldots \ldots \ldots \ldots \ldots \ldots \ldots \ldots \ldots \ldots \ldots$

6.5 Exemplo 04 - Acoplamento cilindro cúpula esférica . . . . . . . . . . . . 79

6.6 Exemplo 05 - Acoplamento tronco de cone placa circular . . . . . . . . . . . 84

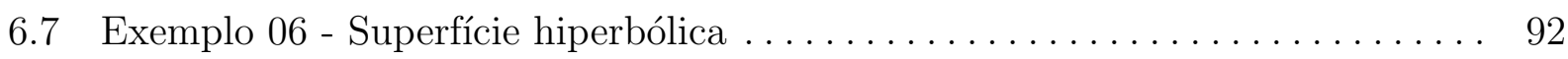

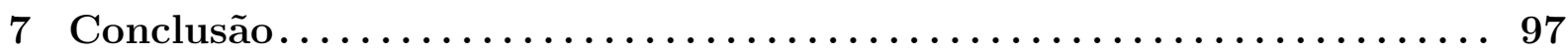

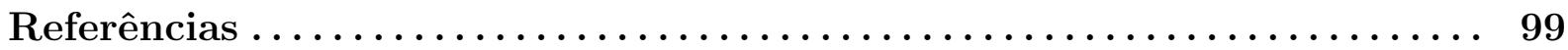




\section{Introdução}

Esta dissertação de Mestrado faz parte da linha de pesquisa de Métodos Numéricos da pós-graduação do departamento de Engenharia de Estruturas da Escola de Engenharia de São Carlos, tendo o apoio financeiro da CAPES (Coordenação de Aperfeiçoamento de Pessoal de Nível Superior).

\subsection{Considerações Iniciais}

O tema da pesquisa foi a implementação do Método dos Elementos Finitos Generalizados (MEFG), de acordo com (DUARTE; BABUSKA; ODEN, 2000), aplicado ao estudo das cascas com simetria de revolução em forma e carregamento, modelados com a teoria cinemática de primeira ordem de Reissner-Mindlin. O MEFG teve sua proposta inicial em (MELENK; BABUSKA, 1996) e (DUARTE; ODEN, 1995). Neste método o espaço de aproximação é construído pelo produto da partição da unidade (PU) com funções que apresentam boas propriedades de aproximação, como as funções polinomiais, que segundo exposto em (MELENK; BABUSKA, 1996) geram bons resultados quando a solução do problema é suficientemente suave.

Na Escola de Engenharia de São Carlos/USP o tema das cascas foi originalmente desenvolvido pelo Prof. Dante A.O. Martinelli, a partir da década de sessenta, particularmente visando o estabelecimento de metodologias de cálculo e projeto de estruturas civis. Naquela época apesar da reduzida disponibilidade de recursos computacionais, já explorava-se a aplicação de técnicas matriciais, porém os cálculos eram, sobretudo, restritos a procedimentos simplificados oriundos da aplicação da Teoria da Elasticidade. Notas de aulas de cursos de graduação e pós-graduação, além de dissertações de mestrado e doutorado, foram o instrumento principal de divulgação dos estudos então realizados, (MARTINELLI, 1983; ALMEIDA, 1982).

Após o afastamento do Prof. Martinelli das atividades didáticas, o tema das cascas teve, em São Carlos, continuidade com trabalhos sobre Coberturas Pênseis e Es- 
truturas Infláveis, (AGUIAR; BARBATO, 2000; OLIVEIRA; BARBATO, 1998, 2002). Além daqueles trabalhos, e procurando-se atender às necessidades de ampliação das linhas de pesquisa da Área, incluindo-se a consideração de não-linearidades, passou-se a dar mais ênfase a tópicos mais específicos relacionados aos campos da Mecânica dos Materiais, (PROEnÇA, 1988) e dos Métodos Numéricos. No campo dos métodos numéricos, a disponibilidade de novos recursos computacionais em conjunto com o desenvolvimento de formas não-convencionais do Método dos Elementos Finitos (BARROS, 2002; PIMENTA; PROENÇA; FREITAS, 2002), tem possibilitado a realização de simulações mais eficientes e a busca de soluções aproximadas mais precisas. Entre as formas não-convencionais do MEF, que tem sido pesquisada, pode-se citar, por exemplo, o Método dos Elementos Finitos Generalizados, MEFG, (BARRos; PROEnÇA; BARCELlos, 2002). O emprego de funcionais modificados de energia com vistas à formulação de elementos finitos de casca livres de travamento (NÓBREGA, 1997) é outro exemplo de pesquisa de interesse no que diz respeito ao desenvolvimento de novas alternativas numéricas.

O Método dos Elementos Finitos Generalizados tem como uma de suas características o enriquecimento nodal das funções de aproximação. Para o enriquecimento adotam-se funções polinomiais associadas aos nós de extremidade do elemento, sendo esse recurso empregado de acordo com a necessidade do problema, ficando opcionais tanto a região a ser enriquecida quanto o grau do polinômio de enriquecimento.

Para o estudo desenvolvido, as cascas podem ter geometria de revolução qualquer, pois o elemento finito utilizado na discretização tem a capacidade de adaptar-se à forma desejada (AHMAD; IRONS; ZIENKIEWICZ, 1970). Estudos sobre o elemento proposto por Ahmad e outros elementos axissimétricos podem ser encontrados em (SORIANO, 2003; ZIENKIEWICZ; R.L.TAYLOR, 2000). Os casos de carregamento previstos neste trabalho são os de pressão uniforme, de peso próprio e pressão hidrostática, todos distribuídos por unidade de superfície. Considera-se ainda a possibilidade de aplicação de forças e momentos concentrados nos extremos dos elementos.

Como ferramenta de trabalho foi desenvolvido um código computacional, em linguagem FORTRAN, para obtenção dos resultados de esforços e deslocamentos.

Para fins de confronto e validação dos resultados obtidos pelo código, foi utilizado um pacote de elementos finitos consagrado e de uso freqüente no meio acadêmico (ANSYS Versão 9.0). 


\subsection{Objetivos}

O objetivo principal deste trabalho foi o desenvolvimento de uma ferramenta numérica para a resolução de problemas de estruturas em casca com simetria de revolução de forma e carregamento. O recurso à alternativa numérica decorre do fato que a formulação em forma forte dos problemas em questão recai em equações diferencias com solução analítica possível em poucos casos de geometria e carregamento simplificado.

A ferramenta numérica consiste em um código computacional, baseado no Método dos Elementos Finitos combinado com técnicas de enriquecimento nodal da função aproximadora.

Como já citado anteriormente, o foco desta pesquisa está nas estruturas em casca, com geometria de revolução qualquer. Outro aspecto também abordado neste trabalho foi o acoplamento, ou a junção, de cascas de diferentes formatos e a obtenção dos resultados, de esforços e deslocamentos nessa situação.

\subsection{Justificativas}

Métodos numéricos são ferramentas bastante eficazes na resolução dos problemas associados à engenharia de estruturas, estando cada vez mais presentes na vida do engenheiro.

Tendo em vista o grau de complexidade da resolução analítica das estruturas de cascas, sob diferentes condições de carregamento, a busca por alternativas numéricas, que se mostrem mais eficientes que as convencionais, se torna bastante interessante e importante. A linha de pesquisa que trata do desenvolvimento de formulações nãoconvencionais, vem ganhando mais importância e sendo cada vez mais difundida. $\mathrm{O}$ Método dos Elementos Finitos Generalizados é entendido como uma ferramenta bastante eficaz nesse sentido.

Os recursos usuais para obtenção de uma melhor aproximação consistem em refino da rede, ou seja, aumentando o número de elementos para discretizar a estrutura, ou em aumento do grau da função polinomial de aproximação. Neste trabalho optou-se por explorar o refino do tipo $p$, em particular mediante o enriquecimento nodal das funções de aproximação. Essa opção permite trabalhar com uma menor quantidade de elementos finitos, obtendo-se melhoria na aproximação ao custo menor de inclusão de novos parâmetros nodais. 


\subsection{Organização do trabalho}

No segundo capítulo desta dissertação são apresentados os comportamentos de membrana e de flexão para as estruturas formadas por casca de revolução, destacando-se efeitos localizados e o amortecimento nos esforços solicitantes, de flexão.

A formulação do elemento finito axissimétrico, utilizado nesta dissertação, é comentada no capítulo 3 deste trabalho.

No quarto capítulo faz-se uma abordagem sobre o Método dos Elementos Finitos Generalizados, a forma de enriquecimento adotada e as possíveis funções para Partição da Unidade sobre o elemento.

A ferramenta computacional desenvolvida nesta pesquisa é mostrada e comentada no quinto capítulo deste trabalho. Nessa parte do texto, comenta-se todas as habilidades que o código possui, os dois tipos de elementos possíveis de serem usados e suas propriedades, casos de carregamento, vinculações e o tipo da função de enriquecimento.

O capítulo 6 traz alguns exemplos propostos e resultados obtidos com o programa desenvolvido. Os problemas envolvem estruturas dos tipos: placas, cascas cilíndricas e esféricas, bem como estruturas formadas pelo acoplamento de cascas de diferentes formas. Nesse capítulo também é apresentada uma análise de erro em relação a uma solução de referência e taxas de convergência resultantes dos refinos do tipo h e do tipo p.

Como último capítulo desta dissertação são apresentadas as conclusões do estudo desenvolvido.

No final do texto, é apresentada toda bibliografia utilizada e consultada durante a execução deste trabalho. 


\section{Cascas de Revolução}

Pode-se entender as estruturas laminares como sendo aquelas que possuem uma dimensão muito menor que as outras. Com isso torna-se possível condensar o estudo do seu comportamento na sua superfície média.

Admitindo a conceituação anteriormente citada, surgem dois casos gerais possíveis para compor a classificação geométrica das superfícies médias: as ditas planas, onde se incluem as estruturas do tipo chapa e placa, sendo elas diferidas entre si apenas pela forma de carregamento e as não-planas, caso das estruturas de casca.

Esta pesquisa se atém apenas ao último tipo de estrutura mencionado, e ainda com restrição ao caso das cascas com simetria de revolução em forma e carregamento.

\subsection{Estruturas Axissimétricas}

Cascas axissimétricas ou de revolução possuem superfície média gerada a partir da rotação de uma curva geratriz em torno de um eixo de referência. Para o carregamento distribuído ao longo da superfície, faz-se também a restrição de axissimetria, isto é, localmente não existe componente de direção tangente ao paralelo.

Como ilustração apresenta-se na figura 1 uma visão tridimensional de uma superfície de revolução e o traço seu num plano meridiano.

\subsection{Hipóteses normalmente usadas em abordagens analíticas sim- plificadas}

Em regime linear, o estudo das superfícies (chapas, placas e cascas) pode ser feito fundamentalmente por aplicação da teoria da elasticidade.

O conjunto de hipóteses simplificadoras normalmente adotado a fim de consti- 

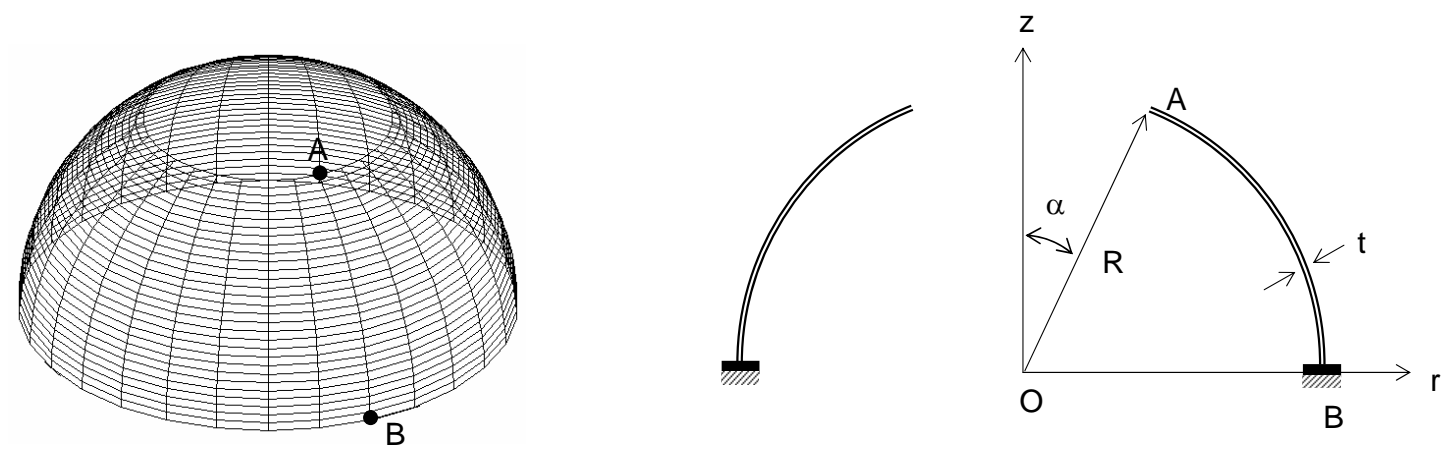

Figura 1: Estrutura espacial e simplificação plana

tuir um modelo matemático dos problemas de valor de contorno incluem as hipóteses cinemáticas de Kirchhoff-Love (GRAVINA, 1957):

1 - O material que constitui o meio é homogêneo, isotrópico e obedece a lei de Hooke;

2 - A espessura da casca é pequena em comparação às demais dimensões e aos raios de curvatura locais da superfície média;

3 - As tensões normais à superfície media são desprezíveis em relação às demais;

4 - Os pontos pertencentes, antes da deformação, à retas normais à superfície média, encontram-se, após a deformação, sobre retas perpendiculares à superfície média deformada;

5 - Os deslocamentos são muito pequenos em relação à espessura, sendo possível desprezar sua influência nas condições de equilíbrio da casca.

O estudo de primeira ordem decorrente da aplicação das hipóteses acima implica em soluções lineares e na possibilidade de utilizar a sobreposição de efeitos, quando necessário.

O presente trabalho tem como enfoque apenas o estudo linear de primeira ordem.

\subsection{Membranas}

Admitindo-se numa primeira abordagem a inexistência de rigidez à flexão e rigidez à torção na casca e que para o equilíbrio ao carregamento aplicado sejam mobilizados apenas esforços internos tangenciais à superfície média, é possível desenvolver uma for- 
mulação simplificada para o estudo dessas estruturas. A essa formulação dá-se o nome de Teoria de Membrana,(PROENÇA, 2005).

Para que uma casca se comporte em regime de membrana é necessária a obediência de algumas restrições, como:

- Espessura muito fina;

- Carregamento externo distribuído ao longo da superfície e com variação suave;

- Vinculação com o meio externo na forma de barras pendulares distribuídas e tangenciais à superfície média da borda da casca.

Como citado anteriormente, as membranas são estruturas de paredes muito finas e com grande flexibilidade. Uma vez submetidas a forças externas elas tendem a se deformar de tal forma a mobilizar apenas os esforços internos tangenciais à superfície média. Assim sendo a superfície média tende a coincidir com a superfície de pressão, o que é análoga à curva funicular de forças utilizada nas estruturas lineares (ZAGOTTIS, 1973).

As membranas de revolução apresentam um comportamento ainda mais simplificado, na medida em que os esforços internos são somente do tipo normal às faces dos elementos alinhadas com os paralelos e meridianos (figura 2).

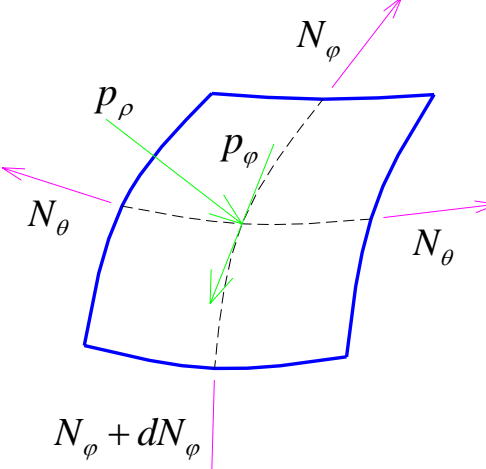

Figura 2: Esforços do regime de membrana

A teoria de membrana em geral leva a resultados com boa representatividade do comportamento estrutural, visto que nas cascas finas os esforços de flexão e torção são de pequena intensidade e localizados em zonas de perturbação do regime de membrana.

Na figura 3 ilustra-se num plano meridiano uma situação de vinculação e carregamento compatíveis com o regime de membrana. 


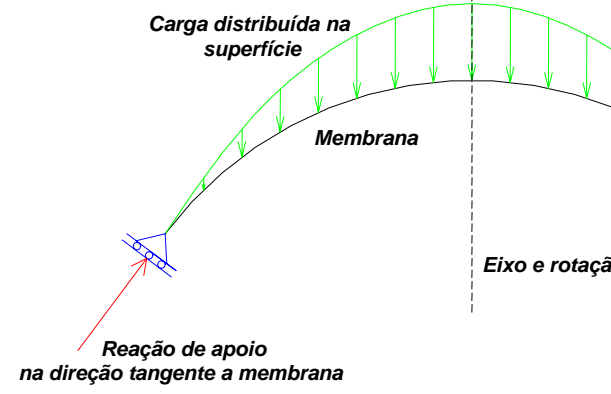

Figura 3: Exemplo de casca de revolução em regime de membrana

\subsection{Equacionamento do regime de membrana}

\subsubsection{Esforços internos}

Considere-se uma casca de revolução, carregada de forma axissimétrica, como já citado anteriormente. Tal configuração leva um problema simplificado do ponto de vista de seu equacionamento.

Seja, agora, um elemento infinitesimal de membrana de revolução sujeito a forças externas e esforços internos, como mostra a figura 4. Nota-se que a simetria de revolução implica em esforço anular $N_{\theta}$ constante ao longo de um paralelo. Tanto os esforços meridionais $\left(N_{\varphi}\right)$ quanto os anulares $\left(N_{\theta}\right)$ possuem dimensão de força por unidade de comprimento

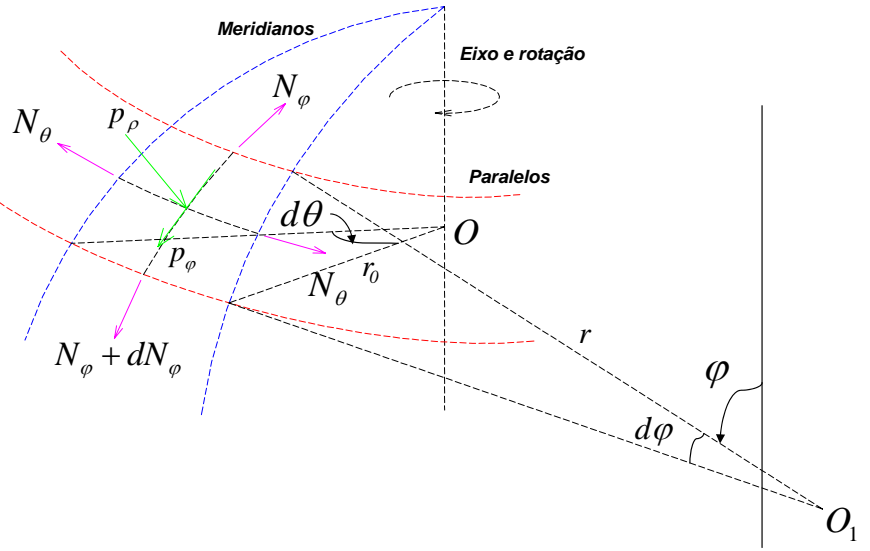

Figura 4: Esforços no elemento infinitesimal de membrana

Para a análise do equilíbrio do elemento é conveniente representar os esforços internos segundo o plano do meridiano e segundo o plano do paralelo, como ilustra a 
figura 5 (PROENÇA, 2005).

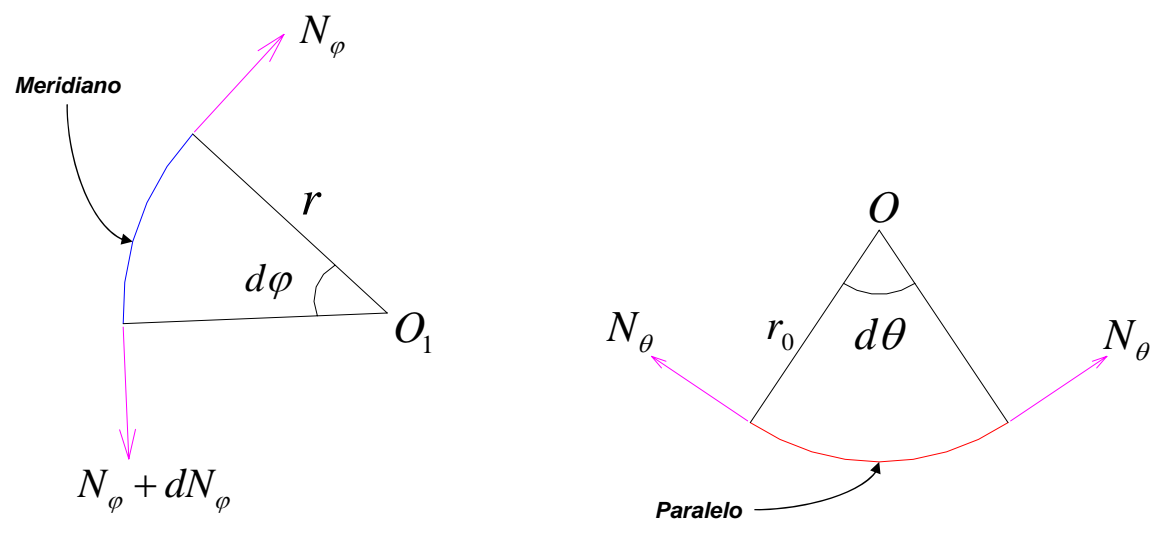

Figura 5: Esforços nos paralelos e meridianos da membrana

Considerando a decomposição do carregamento e dos esforços internos segundo as direções normal e tangencial ao meridiano central do elemento infinitesimal de membrana, pode-se escrever as equações de equilíbrio a partir das considerações que seguem:

A resultante do carregamento segundo a normal à superfície pode ser escrita como:

$$
p_{\rho} \cdot r \cdot d \varphi \cdot r_{0} \cdot d \theta
$$

onde $r$ é o raio do meridiano no ponto central do elemento, $\varphi$ é uma coordenada esférica medida partindo do eixo e $\theta$ é o angulo que posiciona pontos do paralelo em relação a um meridiano arbitrário tomado como origem (ver figura 4).

Quanto aos esforços resultantes nas faces do elemento valem as seguintes relações:

$$
N_{\theta} \cdot r \cdot d \varphi \quad \text { e } \quad N_{\varphi} \cdot r_{0} \cdot d \theta
$$

As componentes dos esforços segundo a direção normal à superfície média valem:

$$
N_{\theta} \cdot r \cdot \operatorname{sen}(\varphi) \cdot d \varphi \cdot d \theta \quad \text { e } \quad N_{\varphi} \cdot r_{0} \cdot d \varphi \cdot d \theta
$$

Aplicando a condição de equilíbrio:

$$
\sum F_{\rho}=0
$$

Sendo $\rho$ a direção normal à superfície e desprezando-se infinitésimos de ordem superior, obtém-se a seguinte equação de equilíbrio: 


$$
p_{\rho} \cdot r \cdot d \varphi \cdot r_{0} \cdot d \theta-N_{\theta} \cdot r \cdot \operatorname{sen}(\varphi) \cdot d \varphi \cdot d \theta-N_{\varphi} \cdot r_{0} \cdot d \varphi \cdot d \theta=0
$$

Fazendo as devidas simplificações vem:

$$
N_{\theta}=\frac{N_{\varphi} \cdot r_{0}}{r \cdot \operatorname{sen}(\varphi)}-\frac{p_{\rho} \cdot r_{0}}{\operatorname{sen}(\varphi)}
$$

Para a determinação completa dos esforços internos faz-se necessária uma segunda equação. A segunda equação de equilíbrio pode ser escrita considerando-se que a resultante $N_{\varphi}$ num certo paralelo deve equilibrar a resultante vertical (segundo o eixo de rotação) do carregamento externo aplicado acima ou abaixo do paralelo (ZAGOTTIS, 1973), com ilustra a figura 6.

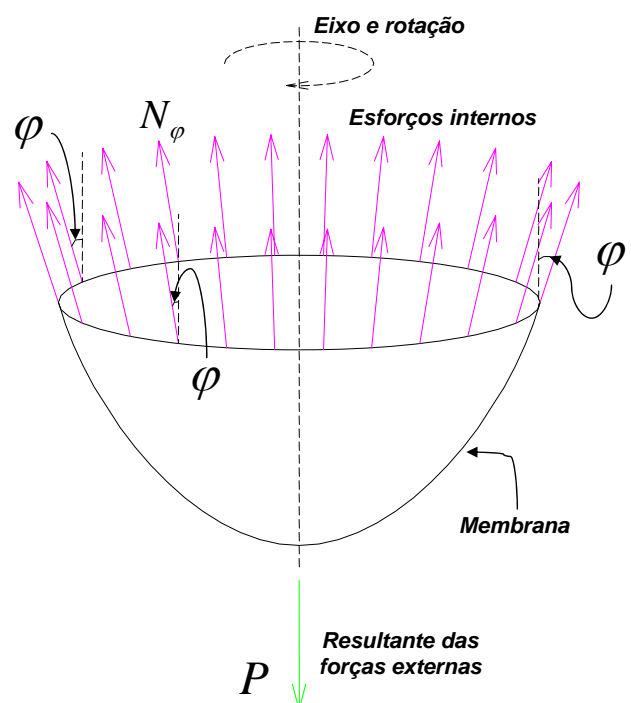

Figura 6: Equilíbrio das forças segundo a direção vertical

A resultante $P$ das forças externas deve passar pelo eixo de rotação da casca, devido às condições de axissimetria anteriormente adotadas, e tem valor determinado por integração das componentes verticais da força externa distribuída por unidade de superfície.

Aplicando a condição de equilíbrio:

$$
\sum F_{V}=0
$$

tem-se como resultado a expressão: 


$$
2 \cdot \pi \cdot r_{0} \cdot N_{\varphi} \cdot \operatorname{sen}(\varphi)=P
$$

Isolando $N_{\varphi}$ na equação anterior, tem-se o valor da força normal na direção da tangente ao meridiano

$$
N_{\varphi}=\frac{P}{2 \cdot \pi \cdot r_{0} \cdot \operatorname{sen}(\varphi)}
$$

Como se pode concluir, o equilíbrio de uma membrana pode ser atingido apenas com a presença de forças internas normais e isso possibilita que essas estruturas tenham um melhor desempenho, podendo-se aproveitar ao máximo a capacidade resistente dos materiais.

\subsubsection{Deslocamentos no regime de membrana}

Os esforços na teoria de membrana ficam definidos apenas com a aplicação das condições de equilíbrio, assim como nas estruturas isostáticas. Para a obtenção dos deslocamentos os esforços internos são relacionados com as deformações, o que é feito a partir da Lei de Hooke, e estas com os deslocamentos por compatibilidade.

As componentes de deformação podem ser obtidas com o auxílio da figura 7, que retrata a configuração inicial e deformada de um elemento infinitesimal da casca segundo o plano meridiano.

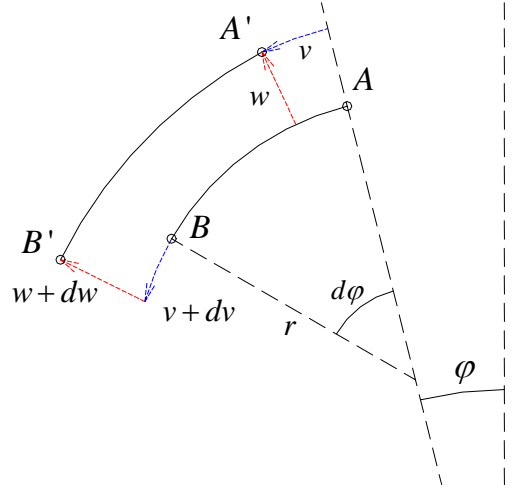

Figura 7: Deslocamentos no regime de membrana

Observando-se a figura 7 é fácil perceber que o deslocamento num ponto da casca pode ser decomposto em duas componentes, transversal e paralela ao raio de curvatura local (PROENÇA, 2005; DINIS, 2004). 
Na direção da tangente ao meridiano uma primeira parcela para a variação do comprimento do arco é dada pelo deslocamento relativo entre os pontos final e inicial do trecho em estudo:

$$
\Delta(A B)_{t}=v+d v-v \quad \Rightarrow \quad \Delta(A B)_{t}=d v
$$

A componente do deslocamento na direção normal à superfície, impõe também, uma variação do comprimento do arco $A B$, proporcional ao aumento do raio de curvatura no ponto considerado:

$$
\Delta(A B)_{n}=(r+w) \cdot d \varphi-r \cdot d \varphi \quad \Rightarrow \quad \Delta(A B)_{n}=w \cdot d \varphi
$$

Dessa forma a componente de deformação da superfície segundo a direção tangente ao meridiano resulta:

$$
\begin{gathered}
\varepsilon_{\varphi}=\frac{\Delta(A B)_{t}+\Delta(A B)_{n}}{A B}=\frac{d v+w \cdot d \varphi}{r \cdot d \varphi} \\
\varepsilon_{\varphi}=\frac{1}{r} \cdot\left(\frac{d v}{d \varphi}+w\right)
\end{gathered}
$$

Analogamente, os paralelos sofrem deformações que estão também vinculadas aos mesmos deslocamentos $v$ e $w$, comentados anteriormente.
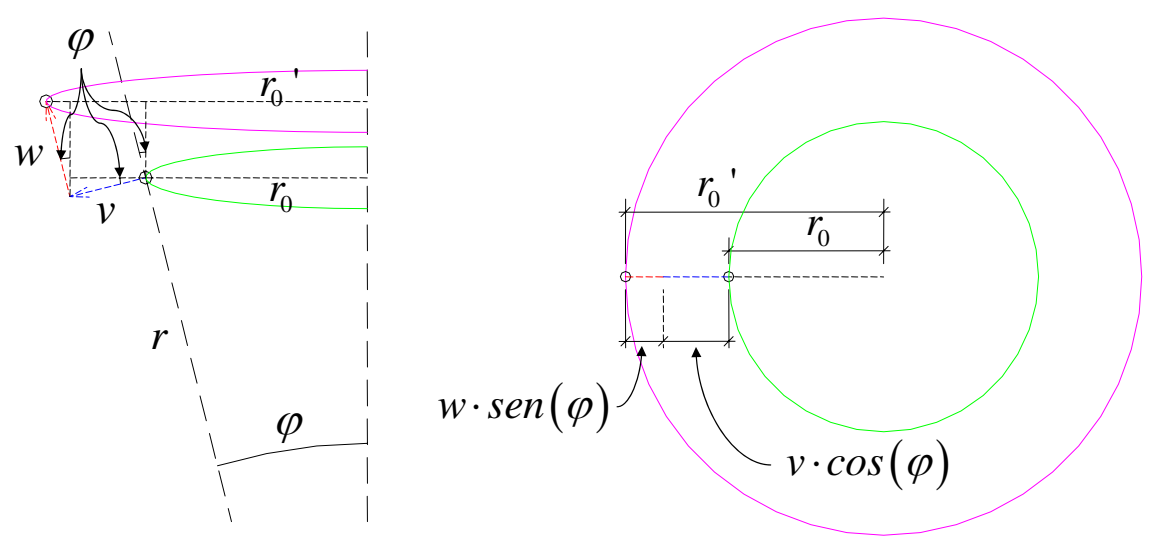

Figura 8: Deformação dos paralelos

Com o auxilio da figura 8 a deformação do paralelo pode ser calculada comparandose a variação do seu comprimento, antes e depois dos deslocamentos: 


$$
\begin{gathered}
\varepsilon_{\theta}=\frac{2 \cdot \pi \cdot r_{0}^{\prime}-2 \cdot \pi \cdot r_{0}}{2 \cdot \pi \cdot r_{0}}=\frac{2 \cdot \pi \cdot\left[r_{0}+v \cdot \cos (\varphi)+w \cdot \operatorname{sen}(\varphi)\right]-2 \cdot \pi \cdot r_{0}}{2 \cdot \pi \cdot r_{0}} \\
\varepsilon_{\theta}=\frac{v}{r_{0}} \cdot \cos (\varphi)+\frac{w}{r_{0}} \cdot \operatorname{sen}(\varphi)
\end{gathered}
$$

Combinando as equações 2.5 e 2.6 de modo a eliminar a componente $w$, obtém-se:

$$
v \cdot \operatorname{cotg}(\varphi)-\frac{d v}{d \varphi}=\frac{\varepsilon_{\theta} \cdot r_{0}}{\operatorname{sen}(\varphi)}-\varepsilon_{\varphi} \cdot r
$$

Da Teoria da Elasticidade é sabido que as tensões e deformações se relacionam como segue:

- Deformações em função das tensões:

$$
\varepsilon_{\varphi}=\frac{1}{E} \cdot\left(\sigma_{\varphi}-\nu \cdot \sigma_{\theta}\right) \quad e \quad \varepsilon_{\theta}=\frac{1}{E} \cdot\left(\sigma_{\theta}-\nu \cdot \sigma_{\varphi}\right)
$$

- Tensões em função das deformações:

$$
\sigma_{\varphi}=\frac{E}{\left(1-\nu^{2}\right)} \cdot\left(\varepsilon_{\varphi}+\nu \cdot \varepsilon_{\theta}\right) \quad e \quad \sigma_{\theta}=\frac{E}{\left(1-\nu^{2}\right)} \cdot\left(\varepsilon_{\theta}+\nu \cdot \varepsilon_{\varphi}\right)
$$

onde $E$ é o módulo de elasticidade longitudinal do material; $\nu$ é o coeficiente de Poisson.

Considerando-se que a casca é muito fina, e com a hipótese de distribuição constante das tensões na espessura (em conformidade com o regime de membrana), a integração das tensões ao longo da espessura, define os esforços internos solicitantes da estrutura:

$$
N_{\varphi}=\int_{-h / 2}^{+h / 2} \sigma_{\varphi} \cdot d \rho=\sigma_{\varphi} \cdot h \quad \rightarrow \quad N_{\theta}=\sigma_{\theta} \cdot h
$$

onde $h$, é a espessura da casca.

Combinando-se as expressões de tensão e deformação com as definições dos esforços internos, obtém-se as seguintes relações para cálculo das deformações em função das forças normais: 


$$
\begin{gathered}
\varepsilon_{\varphi}=\frac{1}{E \cdot h} \cdot\left(N_{\varphi}-\nu \cdot N_{\theta}\right) \\
\varepsilon_{\theta}=\frac{1}{E \cdot h} \cdot\left(N_{\theta}-\nu \cdot N_{\varphi}\right)
\end{gathered}
$$

Substituindo-se as relações 2.8 e 2.9 em 2.7, resulta a seguinte expressão envolvendo o deslocamento na direção da tangente ao meridiano e os esforços internos normais:

$$
v \cdot \operatorname{cotg}(\varphi)-\frac{d v}{d \varphi}=\frac{1}{E \cdot h} \cdot\left\{N_{\varphi} \cdot\left[r+\frac{\nu \cdot r_{0}}{\operatorname{sen}(\varphi)}\right]-N_{\theta} \cdot\left[\frac{r_{0}}{\operatorname{sen}(\varphi)}+\nu \cdot r\right]\right\}
$$

Na expressão anterior, o segundo membro é composto por variáveis já definidas anteriormente em 2.2 e 2.4 e, portanto, por funções dependentes do ângulo $\varphi$. Assim sendo, pode-se referenciar o segundo membro como:

$$
f(\varphi)=\frac{1}{E \cdot h} \cdot\left\{N_{\varphi} \cdot\left[r+\frac{\nu \cdot r_{0}}{\operatorname{sen}(\varphi)}\right]-N_{\theta} \cdot\left[\frac{r_{0}}{\operatorname{sen}(\varphi)}+\nu \cdot r\right]\right\}
$$

Obtém-se assim, uma relação com a seguinte forma:

$$
v \cdot \operatorname{cotg}(\varphi)-\frac{d v}{d \varphi}=f(\varphi)
$$

A resolução da equação 2.10 leva à seguinte forma integral:

$$
v=\left[\int \frac{f(\varphi)}{\operatorname{sen}(\varphi)} d \varphi+C\right] \cdot \operatorname{sen}(\varphi)
$$

Utilizando-se da deformação do paralelo, substituindo a equação 2.11 em 2.6 e utilizando a relação entre deformação e força normal, chega-se à seguinte expressão para o cálculo dos deslocamentos segundo a direção normal à superfície:

$$
w=\frac{\left(N_{\theta}-\nu \cdot N_{\varphi}\right)}{E \cdot h} \cdot \frac{r_{0}}{\operatorname{sen}(\varphi)}-\left[\int \frac{f(\varphi)}{\operatorname{sen}(\varphi)} d \varphi+C\right] \cdot \cos (\varphi)
$$

Vale lembrar que a constante $C$ fica definida a partir de uma condição de contorno relativa aos deslocamentos.

Para obtenção dos deslocamentos segundo um sistema global de coordenadas, atrelado às direções do eixo e normal a ele, $\eta$ e $\xi$, respectivamente, basta aplicar a seguinte 
relação de transformação:

$$
\vec{U}_{g}=J \cdot \vec{U}_{l}
$$

Onde

$$
\vec{U}_{g}=\left(\begin{array}{l}
\xi \\
\eta
\end{array}\right)
$$

é o vetor de deslocamentos referido ao sistema global

$$
\vec{U}_{l}=\left(\begin{array}{c}
v \\
w
\end{array}\right)
$$

é o vetor de deslocamentos referido ao sistema local

$$
J=\left[\begin{array}{cc}
\cos (\varphi) & -\operatorname{sen}(\varphi) \\
\operatorname{sen}(\varphi) & \cos (\varphi)
\end{array}\right]
$$

é a matriz de rotação do sistema de coordenadas.

As relações de transformação resultam:

$$
\begin{aligned}
& \xi=v \cdot \cos (\varphi)-w \cdot \operatorname{sen}(\varphi) \\
& \eta=v \cdot \operatorname{sen}(\varphi)+w \cdot \cos (\varphi)
\end{aligned}
$$

\subsubsection{Rotação da tangente ao meridiano no regime de membrana}

O conjunto de deslocamentos do regime de membrana se completa com a rotação da tangente ao meridiano indicada na figura 9 .

De acordo com o ilustrado na figura 9, o ângulo de rotação sofrido pela reta tangente ao meridiano, é expresso por $\alpha$.

Admitindo-se um regime de pequenas rotações, pode-se confundir o valor da tangente do ângulo com o próprio ângulo. Da figura, obtém-se as seguintes relações: 


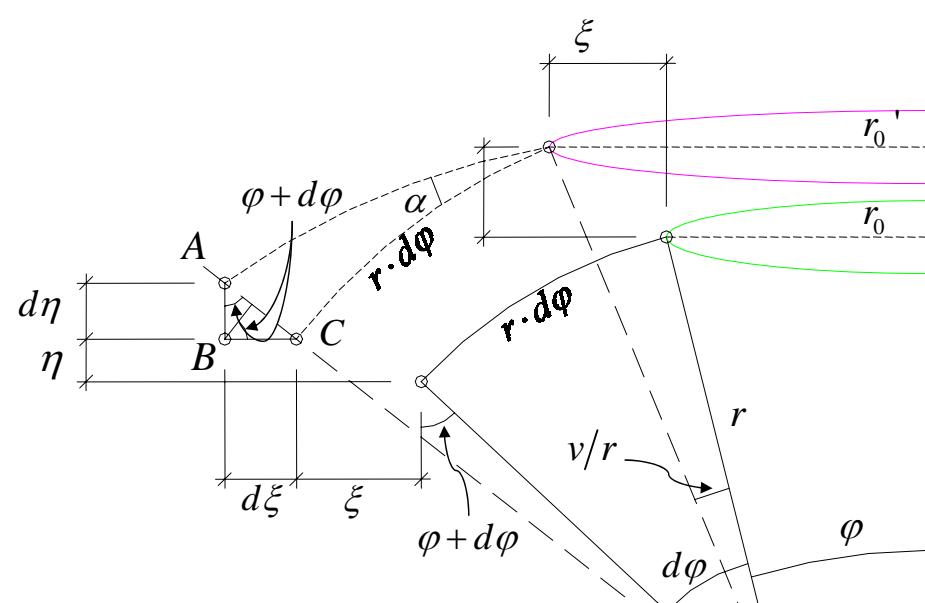

Figura 9: Rotação da tangente ao meridiano

$$
\operatorname{tg}(\alpha) \cong \alpha=\frac{\overline{A C}}{r \cdot d \varphi}
$$

sendo

$$
\overline{A C}=d \xi \cdot \operatorname{sen}(\varphi)-d \eta \cdot \cos (\varphi)
$$

Combinando-se as relações anteriores chega-se a:

$$
\alpha=\frac{1}{r \cdot d \varphi} \cdot[d \xi \cdot \operatorname{sen}(\varphi)-d \eta \cdot \cos (\varphi)]
$$

Substituindo-se na relação anterior, as equações 2.13 e 2.14 e efetuando-se as operações necessárias resulta uma expressão para $\alpha$ em função das componentes de deslocamento segundo as direções tangencial e normal ao meridiano:

$$
\alpha=\frac{1}{r} \cdot\left(\frac{d w}{d \varphi}-v\right)
$$

Em termos gerais, como se pode perceber, a maior dificuldade para obtenção dos deslocamentos e rotação é o cálculo da função $f(\varphi)$ e sua posterior integração. Um fator de complicação se deve ao fato de que $f(\varphi)$ é específica para cada situação, devendo ser determinada para cada tipo de problema. 


\subsection{Cascas em regime de flexão}

O problema das cascas em flexão, já levando em consideração a axissimetria de forma e carregamento, envolve 12 incógnitas básicas, como citado em (GRAVINA, 1957; PROENÇA, 2005):

- Esforços

Momentos fletores: $M_{\varphi}$ e $M_{\theta}$

Força cortante: $Q_{\varphi}$

Forças Normais: $N_{\varphi}$ e $N_{\theta}$

- Deformações e variações de curvatura

Deformações: $\varepsilon_{\varphi}$ e $\varepsilon_{\theta}$

Mudança de curvatura: $\chi_{\varphi}, \chi_{\theta}$

- Deslocamentos:

Tangente ao meridiano: $v$

Normal ao meridiano: $w$

- Rotação:

Tangente ao meridiano: $\alpha$

Para determinação dessas variáveis é necessário um número igual de equações, que resultam das seguintes condições:

- Equações de equilíbrio:

$$
\Sigma F_{\varphi}=0, \quad \Sigma F_{\rho}=0 \quad \text { e } \quad \Sigma M_{\theta}=0
$$

- Equações de compatibilidade:

$$
\begin{aligned}
& \varepsilon_{\varphi}=\varepsilon_{\varphi}(v, w), \quad \varepsilon_{\theta}=\varepsilon_{\theta}(v, w) \quad \text { e } \quad \alpha=\alpha(v, w) \\
& \chi_{\varphi}=\chi_{\varphi}(\alpha) \quad \text { e } \quad \chi_{\theta}=\chi_{\theta}(\alpha)
\end{aligned}
$$

- Equações constitutivas:

$$
\begin{array}{llrl}
\varepsilon_{\varphi}=\varepsilon_{\varphi}\left(N_{\varphi}, N_{\theta}\right) & \text { e } & \varepsilon_{\theta}=\varepsilon_{\theta}\left(N_{\varphi}, N_{\theta}\right) \\
\chi_{\varphi}=\chi_{\varphi}\left(M_{\varphi}, M_{\theta}\right) & \text { e } & \chi_{\theta}=\chi_{\theta}\left(M_{\varphi}, M_{\theta}\right)
\end{array}
$$

Definidas as variáveis do problema das cascas em regime de flexão e as condições 
para sua resolução, mostra-se na seqüência a forma para obtenção das equações componentes do sistema representativo da estrutura.

\subsubsection{Equações de equilíbrio}

A figura 10 mostra os esforços internos para o caso das cascas com simetria de revolução e carregamento axissimétrico.

Em regime de flexão a superfície da casca passa a movimentar momentos fletores, forças de corte, além das forças normais tangenciais (BELLUZZI, 1967).

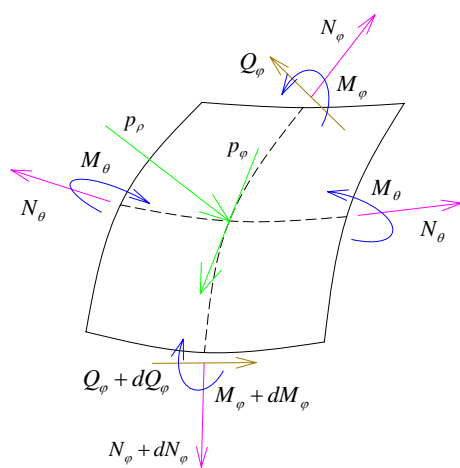

Figura 10: Esforços em regime de flexão

A análise do equilíbrio no regime de flexão é análoga à que foi conduzida para o comportamento de membrana, acrescentando-se a contribuição dos esforços de flexão e corte.

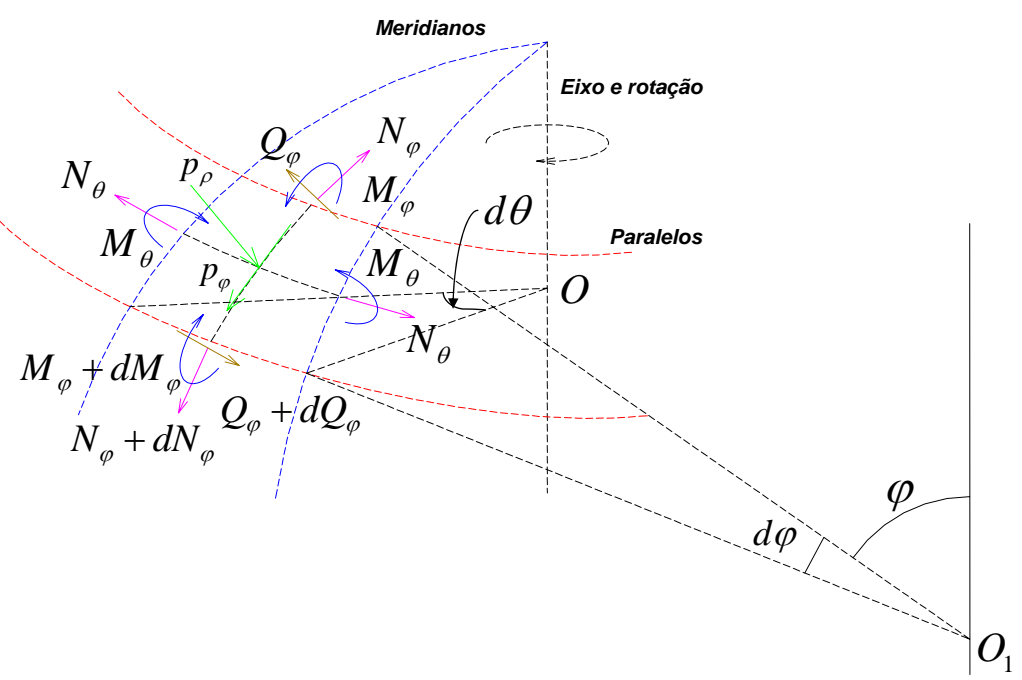

Figura 11: Esforços no elemento infinitesimal em regime de flexão

A figura 11 mostra a configuração equilibrada de um elemento genérico de su- 
perfície média da casca. Na figura 12 apresentam-se vistas em planos paralelos e meridianos, a fim de facilitar a interpretação das contribuições dos esforços nas condições de equilíbrio.
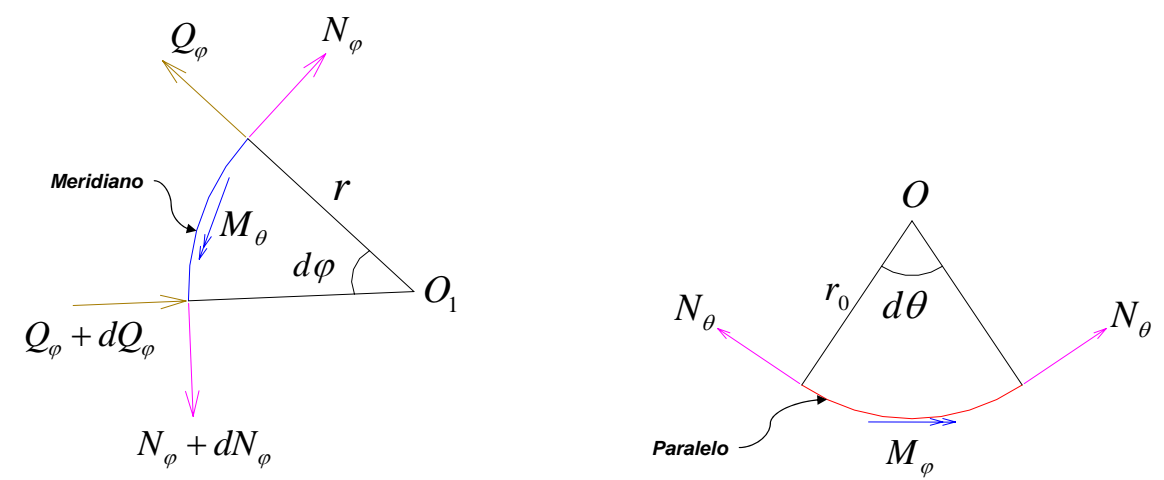

Figura 12: Esforços nos paralelos e meridianos no regime de flexão

Com o auxílio das figuras 11 e 12 escrevem-se três relações de equilíbrio envolvendo os cinco esforços internos. Diferentemente do regime de membrana, o sistema é estaticamente indeterminado (PROENÇA, 2005).

Impondo-se primeiramente que seja nulo o somatório das forças em relação à direção da tangente ao meridiano no ponto médio do elemento,

$$
\Sigma F_{\varphi}=0
$$

após as devidas simplificações obtém-se:

$$
\frac{d}{d \varphi}\left(N_{\varphi} \cdot r_{0}\right)-N_{\theta} \cdot r \cdot \cos (\varphi)-Q_{\varphi} \cdot r_{0}=-p_{\varphi} \cdot r_{0} \cdot r
$$

O equilíbrio de forças na direção da normal à superfície,

$$
\Sigma F_{\rho}=0
$$

fornece:

$$
N_{\varphi} \cdot r_{0}+N_{\theta} \cdot r \cdot \operatorname{sen}(\varphi)+\frac{d}{d \varphi}\left(Q_{\varphi} \cdot r_{0}\right)=-p_{\rho} \cdot r_{0} \cdot r
$$

Finalmente, o equilíbrio de momentos em torno da tangente ao paralelo, 


$$
\Sigma M_{\theta}=0
$$

tem a contribuição dos momentos fletores $M_{\theta}$ e $M_{\varphi}$, exprimindo-se pela seguinte equação:

$$
\frac{d}{d \varphi}\left(M_{\varphi} \cdot r_{0}\right)-M_{\theta} \cdot r \cdot \cos (\varphi)-Q_{\varphi} \cdot r_{0} \cdot r=0
$$

\subsubsection{Relações constitutivas}

Como já comentado, admite-se que as estruturas aqui estudadas têm comportamento elástico-linear regido pela Lei de Hooke. Além disso, a hipótese cinemática sobre a deformação de retas normais à superfície média permite admitir distribuições lineares para as tensões normais e de cisalhamento ao longo da espessura. Assim sendo, são válidas seguintes relações entre esforços e deformações (TIMOSHENKO; GOODIER, 1980):

- Relações entre forças normais e deformações

$$
\begin{aligned}
& N_{\varphi}=\frac{E \cdot h}{1-\nu^{2}} \cdot\left(\varepsilon_{\varphi}+\nu \cdot \varepsilon_{\theta}\right) \\
& N_{\theta}=\frac{E \cdot h}{1-\nu^{2}} \cdot\left(\varepsilon_{\theta}+\nu \cdot \varepsilon_{\varphi}\right)
\end{aligned}
$$

- Relações entre momentos fletores e variações da curvatura

$$
\begin{aligned}
& M_{\varphi}=-D \cdot\left(\chi_{\varphi}+\nu \cdot \chi_{\theta}\right) \\
& M_{\theta}=-D \cdot\left(\chi_{\theta}+\nu \cdot \chi_{\varphi}\right)
\end{aligned}
$$

sendo $D$ nas equações 2.23 e 2.24 a rigidez a flexão de estruturas de superfície (MARTINELLI; MONTANARI; SAVASSI, 2003):

$$
D=\frac{E \cdot h^{3}}{12 \cdot\left(1-\nu^{2}\right)}
$$




\subsubsection{Relações de compatibilidade}

As relações de compatibilidade fazem a ligação entre as deformações e os deslocamentos da estrutura. Entre elas estão as equações 2.5 e 2.6, que relacionam as deformações $\varepsilon_{\varphi}$ e $\varepsilon_{\theta}$ aos deslocamentos normais e tangentes à superfície média.

Restam, agora, as relações de compatibilidade entre as variações de curvatura com os deslocamentos da estrutura, relações essas que podem ser obtidas com base nas figuras 13 e 14 .

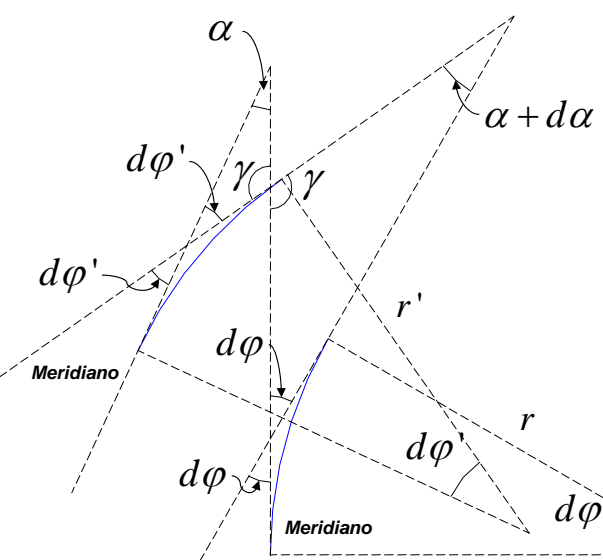

Figura 13: Variação da curvatura tangente ao meridiano

Analisando a figura 13 e desprezando a mudança no comprimento do arco, tiramse as seguintes relações:

$$
d s=r \cdot d \varphi=r^{\prime} \cdot d \varphi^{\prime} \quad \Rightarrow \quad d \varphi^{\prime}=d \varphi+d \alpha
$$

Com a definição de mudança de curvatura segundo o meridiano e utilizando as expressões anteriores, tem-se:

$$
\chi_{\varphi}=\frac{1}{r^{\prime}}-\frac{1}{r}=\frac{1}{r} \cdot \frac{d \alpha}{d \varphi}
$$

ou, então, substituindo-se a equação 2.17 na última expressão, chega-se a:

$$
\chi_{\varphi}=\frac{1}{r} \cdot \frac{d}{d \varphi}\left[\frac{1}{r} \cdot\left(\frac{d w}{d \varphi}-v\right)\right]
$$

que representa a mudança da curvatura em função dos deslocamentos.

Faz-se, agora, a partir da figura 14, uma análise da curvatura segundo a direção 
normal ao meridiano.

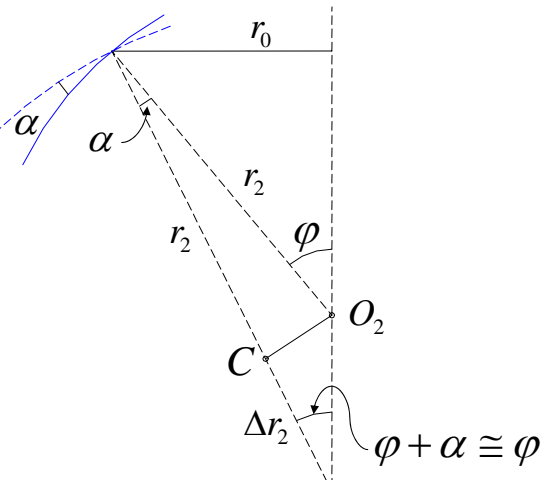

Figura 14: Variação da curvatura na direção normal ao meridiano

Tem-se, então, as seguintes relações:

$$
\begin{gathered}
\overline{O_{2} C}=r_{2} \cdot \alpha=\frac{r_{0}}{\operatorname{sen}(\varphi)} \cdot \alpha \\
\operatorname{tg}(\varphi+\alpha) \cong \operatorname{tg}(\varphi)=\frac{r_{2} \cdot \alpha}{\Delta r_{2}} \quad \Rightarrow \quad \Delta r_{2}=r_{2} \cdot \alpha \cdot \operatorname{cotg}(\varphi)
\end{gathered}
$$

Operando com as expressões anteriores e com a definição da variação de curvatura, resulta:

$$
\chi_{\theta}=\frac{1}{r_{2}^{\prime}}-\frac{1}{r_{2}}=\frac{\Delta r_{2}}{r_{2}^{2}}=\frac{\alpha \cdot \operatorname{cotg}(\varphi)}{r_{2}}=\frac{\alpha \cdot \cos \varphi}{r_{0}}
$$

Substituindo-se a equação que relaciona a rotação $\alpha$ com os deslocamentos e simplificando, obtém-se a relação desejada:

$$
\chi_{\theta}=\frac{\cos \varphi}{r_{0}} \cdot\left[\frac{1}{r} \cdot\left(\frac{d w}{d \varphi}-v\right)\right]
$$

Com o exposto, completa-se o sistema que relaciona todas as variáveis envolvidas no problema de cascas com axissimetria de forma e carregamento.

\subsubsection{Sistema de equações}

Como visto nos itens anteriores, as doze variáveis que compõem o problema podem ser relacionadas entre si. Trabalhando algebricamente com as equações, de acordo 
com (GRAVINA, 1957), é possível combiná-las de forma a reduzir a ordem do sistema de doze para dois, ou seja, colocando-se as demais incógnitas em função de apenas duas. Nesse sentido, escolhe-se as que levam ao menor esforço algébrico. O par mais favorável sugerido na bibliografia é o que envolve o esforço cortante $Q_{\varphi}$ e a rotação do meridiano $\alpha$, (PROENÇA, 2005).

Para exemplificar o exposto no parágrafo anterior, considera-se o caso da casca esférica de espessura constante. Com as devidas combinações, o sistema reduzido toma a seguinte forma:

$$
\begin{gathered}
\frac{d^{2} Q_{\varphi}}{d \varphi^{2}}+\frac{d Q_{\varphi}}{d \varphi} \cdot \operatorname{cotg}(\varphi)-Q_{\varphi} \cdot \operatorname{cotg}^{2}(\varphi)+\nu \cdot Q_{\varphi}=-E \cdot h \cdot \alpha+R \cdot\left[(1-\nu) \cdot p_{\varphi}-\frac{d p_{z}}{d \varphi}\right] \\
\frac{d^{2} \alpha}{d \varphi^{2}}+\frac{d \alpha}{d \varphi} \cdot \operatorname{cotg}(\varphi)-\alpha \cdot \operatorname{cotg}^{2}(\varphi)+\nu \cdot \alpha=\frac{R^{2}}{D} \cdot Q_{\varphi}
\end{gathered}
$$

Nas relações $R$ representa o raio da esfera, $h$ sua espessura, e $D$ a rigidez à flexão.

A solução geral do sistema é composta de duas partes: a homogênea, solução do sistema formado apenas pelos termos associados com as incógnitas $Q_{\varphi}$ e $\alpha$, e a solução particular, ou funções quaisquer para $Q_{\varphi}$ e $\alpha$ que substituídas no sistema reproduzem os termos do carregamento externo.

Cabe agora fazer uma análise da solução do sistema homogêneo:

$$
\begin{gathered}
\frac{d^{2} Q_{\varphi}}{d \varphi^{2}}+\frac{d Q_{\varphi}}{d \varphi} \cdot \operatorname{cotg}(\varphi)-Q_{\varphi} \cdot \operatorname{cotg}^{2}(\varphi)+\nu \cdot Q_{\varphi}=-E \cdot h \cdot \alpha \\
\frac{d^{2} \alpha}{d \varphi^{2}}+\frac{d \alpha}{d \varphi} \cdot \operatorname{cotg}(\varphi)-\alpha \cdot \operatorname{cotg}^{2}(\varphi)+\nu \cdot \alpha=\frac{R^{2}}{D} \cdot Q_{\varphi}
\end{gathered}
$$

Essencialmente, o sistema homogêneo traz consigo os efeitos provenientes das condições de contorno, seja na forma de vínculos ou de eventuais forças aplicadas às bordas da casca.

Levando-se em consideração a natureza exponencial da solução, nota-se nas equações 2.29 e 2.30 que as parcelas relativas à segunda derivada são muito maiores que as demais. Assim, em termos gerais para as variáveis envolvidas: 


$$
\frac{d^{2}(\ldots)}{d \varphi^{2}}>>\frac{d(\ldots)}{d \varphi}>>(\ldots)
$$

Sendo assim é possível desprezar essas parcelas e simplificar o sistema de equações para a seguinte forma:

$$
\begin{gathered}
\frac{d^{2} Q_{\varphi}}{d \varphi^{2}}=-E \cdot h \cdot \alpha \\
\frac{d^{2} \alpha}{d \varphi^{2}}=\frac{R^{2}}{D} \cdot Q_{\varphi}
\end{gathered}
$$

O sistema pode ainda ser reduzido a uma única equação, envolvendo apenas uma variável

$$
\frac{d^{4} Q_{\varphi}}{d \varphi^{4}}=-\left(E \cdot h \cdot \frac{R^{2}}{D}\right) \cdot Q_{\varphi}
$$

Definindo-se

$$
E \cdot h \cdot \frac{R^{2}}{D}=4 \cdot \gamma^{4}
$$

chega-se, finalmente, à equação que deve ser resolvida, a fim de se obter a solução do sistema homogêneo:

$$
\frac{d^{4} Q_{\varphi}}{d \varphi^{4}}+4 \cdot \gamma^{4} \cdot Q_{\varphi}=0
$$

A função solução do sistema homogêneo tem a seguinte configuração:

$$
Q_{\varphi}=e^{\gamma \varphi} \cdot\left[C_{1} \cdot \operatorname{sen}(\gamma \cdot \varphi)+C_{2} \cdot \cos (\gamma \cdot \varphi)\right]+e^{-\gamma \varphi} \cdot\left[C_{3} \cdot \operatorname{sen}(\gamma \cdot \varphi)+C_{4} \cdot \cos (\gamma \cdot \varphi)\right]
$$

As constantes que aparecem na equação 2.34 podem ser determinadas de acordo com as condições de contorno do problema. Em particular, para cascas delgadas $C_{1}=$ $C_{2}=0$ (BELLUZZI, 1967).

Analisando a equação 2.34, nota-se semelhança com a solução que rege o com- 
portamento das vigas sobre base elástica (ZAGOTTIS, 1973).

A figura 15 tenta retratar a semelhança do problema das cascas, com o das vigas sobre base elástica, tomando-se em particular um tubo sob pressão interna.

Nesse caso, os paralelos deformando-se acabam funcionando como uma espécie de base elástica para os meridianos. Como conseqüência, os esforços localizados de flexão provenientes da vinculação decrescem rapidamente, tendendo para valores muito pequenos nas regiões afastadas dos apoios, ou das bordas com cargas aplicadas. Assim, longe das bordas os efeitos significativos, remanescentes, são os de membrana.

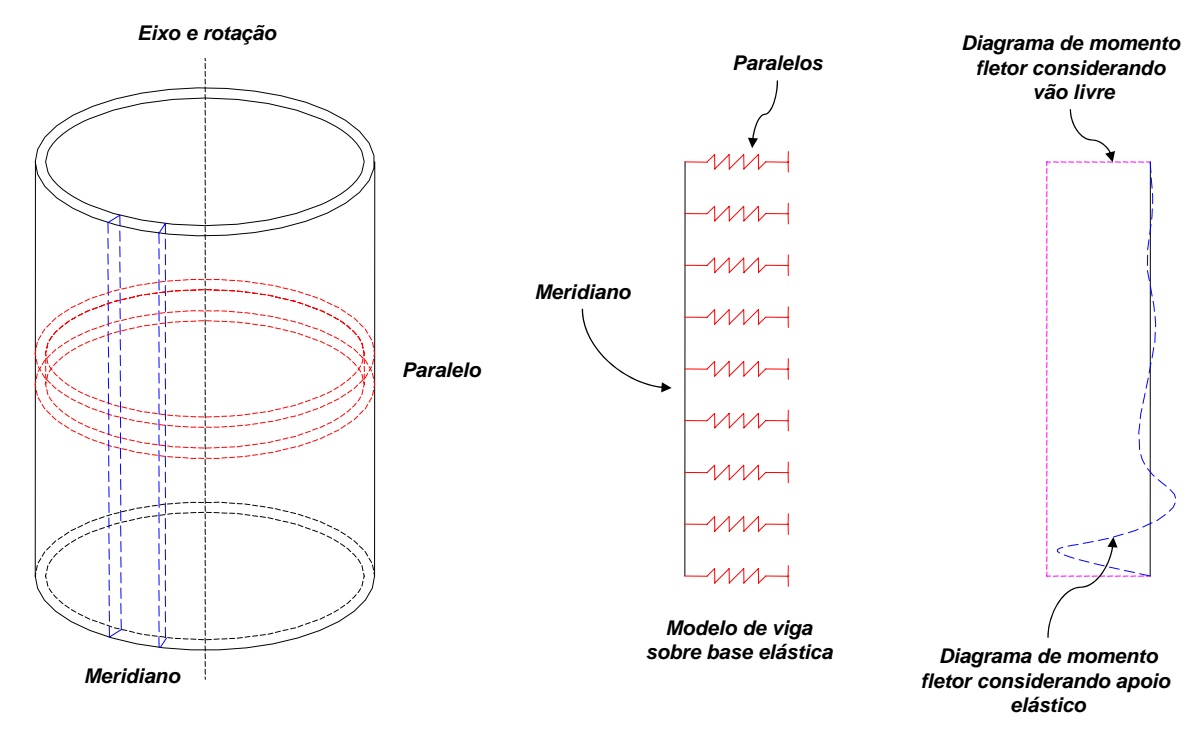

Figura 15: Comparação entre cascas e viga sobre base elástica

Voltando à solução (2.34) são os termos do tipo $e^{-k \cdot x}$ que são responsáveis pelo amortecimento.

A solução particular depende do tipo do carregamento externo, mas independente das cargas atuantes, pode-se mostrar que expressões particulares para $Q_{\varphi}$ apresentam variação proporcional à relação $(h / R)$. Isso significa que para uma casca de pequena espessura em relação ao raio, os esforços de flexão (momentos fletores e força cortante) tendem para zero, sendo significativos no estado particular apenas os esforços normais.

Pode-se, então, adotar como solução particular para o problema em estudo a solução de membrana.

Uma última solução simplificada deriva da chamada Aproximação de Geckeler (GRAVINA, 1957), para cascas de revolução de forma qualquer. Admite-se que a solução do sistema homogêneo coincide com a de uma casca esférica "inscrita", que tenha retas tangentes à borda concordantes com as da casca geral em estudo. 
Deve-se ressaltar que a Aproximação de Geckeler é valida para as superfícies de revolução em geral, independente de sua forma. A restrição que se faz à essa simplificação é sua aplicação para as cascas abatidas, pois nestas o efeito do amortecimento é pouco significativo.

Para se obter o resultado final de esforços e deslocamentos, deve-se somar as soluções dos sistemas particular (regime de membrana) e homogêneo.

\subsection{Considerações complementares}

O objetivo principal deste capítulo foi o de enfatizar aspectos gerais do comportamento das cascas, particularmente os regimes de membrana (predominante) e de flexão (localizado).

Há de se notar também que a flexão localizada se traduz em distribuições de momentos e cortantes de intensidade variável e com inversão de sinal. Esse fato não é de fácil reprodução quando do emprego de técnicas numéricas e, com freqüência, pouco ressaltado nos trabalhos que tratam da aplicação de métodos numéricos.

No que segue, passa-se à consideração de uma metodologia numérica para a análise de cascas de revolução sobre condições mais gerais de geometria e carregamento. Os resultados numéricos a serem apresentados terão como objetivo enfatizar os aspectos conceituais apresentados neste capítulo. 


\section{Elemento Finito Axissimétrico}

Neste capitulo é estudado o elemento finito utilizado para elaboração do código computacional que será mostrado numa etapa futura desta dissertação. Neste ponto, a abordagem do elemento se refere à sua formulação do ponto de vista do Método dos Elementos Finitos convencional.

A teoria de cascas descrita no capítulo 2 se refere ao modelo clássico de Kirchoff - Love para cascas finas, onde não são considerados os efeitos da deformação cisalhante.

Formulações numéricas não-convencionais para cascas baseadas nas hipóteses de Kirchoff - Love, podem ser encontradas em trabalhos como: (ALVES, 2005), que aplicou o Método dos Mínimos Quadrados à resolução de tubos cilíndricos, e em (NIRSCHL, 2005) que aplica técnicas de enriquecimento das funções de aproximação do elemento finito unidimensional para a resolução de cascas cilíndricas e esféricas. Para o presente trabalho, diferentemente dos anteriores, optou-se pelo modelo de primeira ordem de Reissner Mindlin, onde se considera o efeito da força cortante sobre a deformação da reta normal, fato esse que se torna significativo quando a estrutura deixa de ser considerada fina.

Para o desenvolvimento deste trabalho utiliza-se do elemento sólido degenerado de Ahmad, (SORIANO, 2003; ZIENKIEWICZ; R.L.TAYLOR, 2000) com geometria, aqui definida, em coordenadas cilíndricas. Cabe também comentar que o elemento em estudo, restrito ao caso da axissimetria, é composto por 3 nós, tendo a cada um deles 3 graus de liberdade, dois deslocamentos e uma rotação.

A apresentação do elemento será feita na seguinte seqüência, a fim de auxiliar a compreensão do seu funcionamento:

- Características geométricas e o emprego da função de mapeamento;

- Construção do modelo cinemático de primeira ordem;

- Equações constitutivas para materiais homogêneos, isotrópicos e com comportamento elástico linear; 
- Formulação fraca obtida por resíduos ponderados (Galerkin);

- Discretização do problema e as equações de Bubnov - Galerkin.

A descrição do conteúdo deste capítulo segue, em grande parte, de acordo com as referências (SUAREZ, 2003) e (SUAREZ; PROENÇA, 2006).

\subsection{Características geométricas do elemento}

Como o estudo é referido às cascas de revolução com carregamento axissimétrico, o elemento sólido tridimensional pode ter sua representação restrita a um plano, caracterizandose a linha de sua interseção com a superfície média e a espessura da casca ao longo dela. O posicionamento global de pontos no plano considerado é feito com a utilização do sistema cilíndrico. A geometria qualquer da linha representativa da superfície média pode ser construída mediante mapeamento a partir de um domínio paramétrico.

\subsubsection{Base ortonormal associada a um nó do elemento}

Ao nó $k$ do elemento pode-se associar uma base ortonormal, composta pelos seguintes versores: $\left[\vec{v}_{2 k}: \vec{v}_{3 k}: \vec{i}_{\theta}\right]$. Tais versores estão mostrados na figura 16 e podem ser definidos da forma descrita a seguir.

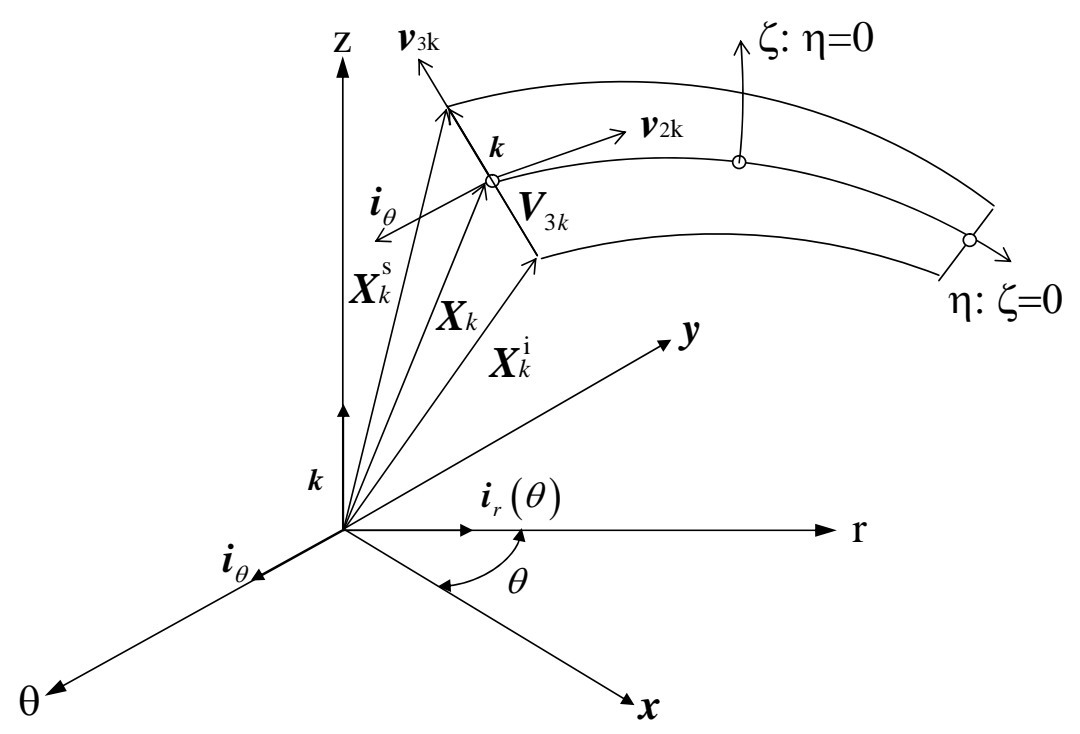

Figura 16: Base ortonormal associada a um nó do elemento

Primeiramente forma-se o vetor $\vec{V}_{3 k}$, que tem módulo igual à espessura local da casca e direção dada por: 


$$
\vec{V}_{3 k}=\vec{X}_{k}^{s}-\vec{X}_{k}^{i}
$$

sendo $\vec{X}_{k}^{s}$ e $\vec{X}_{k}^{i}$ os vetores posição dos pontos que compõem as extremidades locais da espessura da casca.

Segue então o versor:

$$
\vec{v}_{3 k}=\frac{\vec{V}_{3 k}}{\left|\vec{V}_{3 k}\right|}
$$

Como o vetor $\vec{V}_{3 k}$ tem módulo igual à espessura $t_{k}$ do elemento, no ponto considerado, pode-se escrevê-lo da seguinte maneira:

$$
\vec{V}_{3 k}=\vec{v}_{3 k} \cdot t_{k}
$$

O segundo versor, $\left(\vec{i}_{\theta}\right)$, é ortogonal ao plano $(r-z)$, como mostrado na figura 16 . A base se completa com o versor resultante do produto vetorial entre os versores $\vec{v}_{3 k}$ e $\vec{i}_{\theta}$ :

$$
\vec{v}_{2 k}=\vec{v}_{3 k} \times \vec{i}_{\theta}
$$

\subsubsection{Mapeamento na definição da geometria do elemento}

A posição de um ponto qualquer no interior do elemento pode ser definida por mapeamento, empregando funções de interpolação, e deve obedecer à relação:

$$
\vec{X}=\vec{X}(\eta, \zeta, \theta), X: \Omega_{e} \rightarrow R^{3}
$$

onde $\Omega_{e}$ é o domínio paramétrico do elemento

$$
\Omega_{e}=\{\eta, \zeta, \theta \in R /-1 \leq \eta \leq 1,-1 \leq \zeta \leq 1,0 \leq \theta \leq 2 \pi\}
$$

A interpolação utilizada para representação dos pontos no interior do elemento é a linear na direção do eixo paramétrico $\zeta$ e quadrática em $\eta$, sendo usadas para tal as 


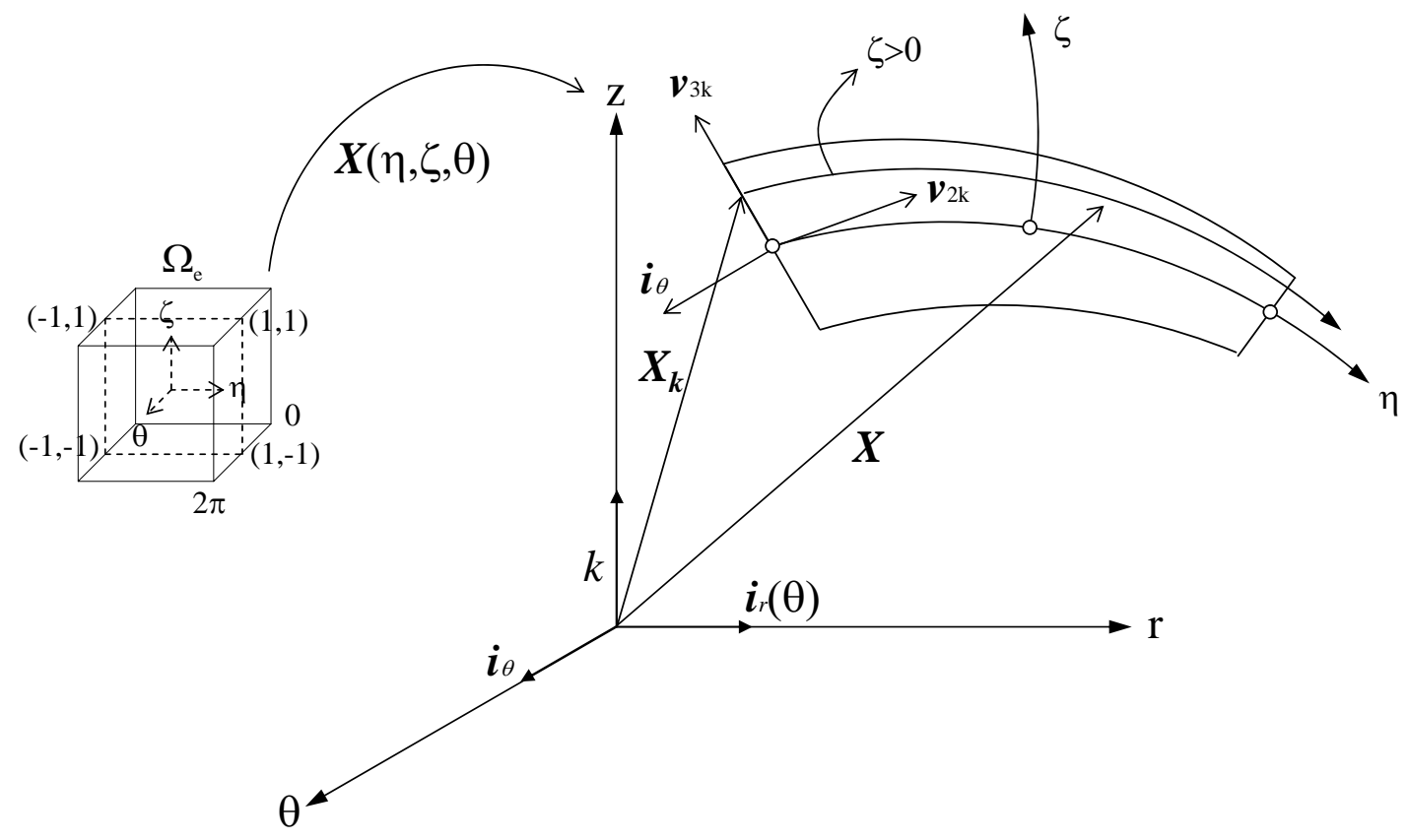

Figura 17: Domínio Paramétrico e Vetor posição

seguintes funções lagrangianas:

- Funções lineares

$$
g_{s}(\zeta)=\frac{1}{2} \cdot(1+\zeta) \quad e \quad g_{i}(\zeta)=\frac{1}{2} \cdot(1-\zeta)
$$

- Funções quadráticas

$$
f_{1}(\eta)=\frac{1}{2} \cdot\left(\eta^{2}-\eta\right) \quad ; \quad f_{2}(\eta)=1-\eta^{2} \quad \text { e } \quad f_{3}(\eta)=\frac{1}{2} \cdot\left(\eta^{2}+\eta\right)
$$

Para um ponto qualquer ao longo da espessura associada ao nó $k$, direcionada com o versor $\vec{v}_{3 k}$, a expressão para sua posição por interpolação dos vetores $\vec{X}_{k}^{i}$ e $\vec{X}_{k}^{s}$ assume a forma:

$$
\vec{X}_{k}(\zeta, \theta)=\vec{X}_{k}^{s}(\theta) \cdot g_{s}(\zeta)+\vec{X}_{k}^{i}(\theta) \cdot g_{i}(\zeta)
$$

Substituindo-se as funções lagrangianas e reorganizando de forma mais conveniente, obtém-se:

$$
\vec{X}_{k}(\zeta, \theta)=\frac{1}{2} \cdot\left[\vec{X}_{k}^{s}(\theta)+\vec{X}_{k}^{i}(\theta)\right]+\frac{1}{2} \cdot\left[\vec{X}_{k}^{s}(\theta)-\vec{X}_{k}^{i}(\theta)\right] \cdot \zeta
$$


Utilizando a expressão 3.2 e definindo-se a média entre $\vec{X}_{k}^{s}(\theta)$ e $\vec{X}_{k}^{i}(\theta)$ como $\vec{X}_{k, \text { med }}(\theta)$, a equação anterior toma a seguinte forma:

$$
\vec{X}_{k}(\zeta, \theta)=\vec{X}_{k, \text { med }}(\theta)+\vec{v}_{3 k}(\theta) \cdot \frac{t_{k}}{2} \cdot \zeta
$$

Expressões análogas à 3.4 podem ser construídas para fornecer as posições de quaisquer pontos sobre as espessuras atreladas aos outros nós do elemento. Finalmente pode-se chegar também por interpolação a uma expressão final para a posição de qualquer ponto no interior do elemento considerando que ele pertença a uma linha dada por $\zeta=$ cte. Neste sentido, basta associar às equações do tipo 3.4 a interpolação quadrática ligada ao eixo $\eta$ :

$$
\vec{X}(\eta, \zeta, \theta)=\sum_{k=1}^{n} f_{k}(\eta) \cdot \vec{X}_{k}(\zeta, \theta)
$$

Desenvolvendo a expressão anterior e considerando-se que em cada nó o versor $\vec{v}_{3 k}$ se decompõem segundo as direções dos versores globais $\vec{i}_{r}(\theta)$ e $\vec{k}$ empregando-se os cossenos diretores do ângulo varphi entre o eixo $r$ e o próprio versor, a equação final para interpolação dos pontos no interior do elemento tem a seguinte configuração:

$$
\begin{gathered}
\vec{X}(\eta, \zeta, \theta)=\left[\sum_{k=1}^{n} f_{k}(\eta) \cdot r_{k}+\sum_{k=1}^{n} f_{k}(\eta) \cdot v_{3 k}^{r} \cdot \frac{t_{k}}{2} \cdot \zeta\right] \cdot \vec{i}_{r}(\theta)+ \\
{\left[\sum_{k=1}^{n} f_{k}(\eta) \cdot z_{k}+\sum_{k=1}^{n} f_{k}(\eta) \cdot v_{3 k}^{z} \cdot \frac{t_{k}}{2} \cdot \zeta\right] \cdot \vec{k}}
\end{gathered}
$$

sendo $n$ o número de nós do elemento.

Reescrevendo-se a equação 3.5, numa forma reduzida, em coordenadas cilíndricas, chega-se a

$$
\vec{X}(\eta, \zeta, \theta)=r(\eta, \zeta) \cdot \vec{i}_{r}(\theta)+z(\eta, \zeta) \cdot \vec{k}
$$

Assim, com a equação 3.6, o mapeamento do domínio intrínseco do elemento, para o domínio real do problema, fica perfeitamente definido.

\subsubsection{Base co-variante e ortonormal para um ponto qualquer do elemento}

Definido o vetor posição de um ponto genérico no interior do elemento, é possível agora, determinar os vetores tangentes ao trio de linhas paramétricas $\eta, \zeta$ e $\theta$, associados 
a esse ponto. A esse terno de vetores, dá-se o nome de base co-variante. Vale lembrar que o conjunto citado não necessariamente tem seus componentes perpendiculares entre si.

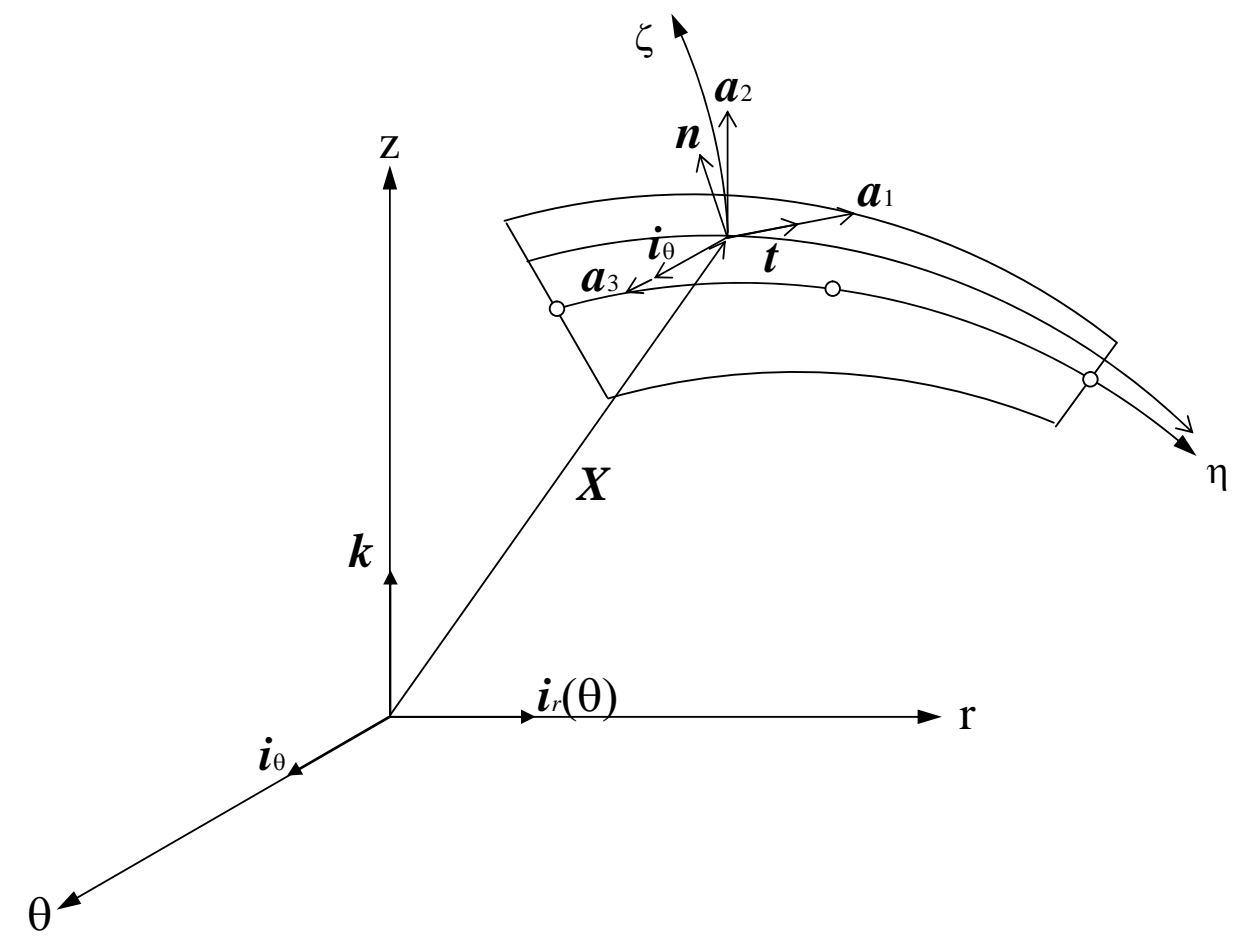

Figura 18: Bases co-variante e ortonormal para um ponto qualquer do elemento

Como forma de representação para a base co-variante, será utilizada a seguinte notação matricial; reunindo em três colunas as componentes dos vetores $\vec{a}_{1}, \vec{a}_{2}$ e $\vec{a}_{3}$ :

$$
\left[F_{\zeta}\right]=\left[\vec{a}_{1}, \vec{a}_{2}, \vec{a}_{3}\right]
$$

Cada vetor da base em questão é definido como segue

$$
\begin{gathered}
\vec{a}_{1}=\frac{\partial \vec{X}}{\partial \eta}=\frac{\partial}{\partial \eta}\left[r(\eta, \zeta) \cdot \vec{i}_{r}(\theta)\right]+\frac{\partial}{\partial \eta}[z(\eta, \zeta) \cdot \vec{k}] \\
\vec{a}_{2}=\frac{\partial \vec{X}}{\partial \zeta}=\frac{\partial}{\partial \zeta}\left[r(\eta, \zeta) \cdot \vec{i}_{r}(\theta)\right]+\frac{\partial}{\partial \zeta}[z(\eta, \zeta) \cdot \vec{k}] \\
\vec{a}_{3}=\frac{\partial \vec{X}}{\partial \theta}=\frac{\partial}{\partial \theta}\left[r(\eta, \zeta) \cdot \vec{i}_{r}(\theta)\right]+\frac{\partial}{\partial \theta}[z(\eta, \zeta) \cdot \vec{k}]=r(\eta, \zeta) \cdot \vec{i}_{\theta}
\end{gathered}
$$

Equacionando as expressões citadas acima, tendo em vista a equação 3.5, obtém- 
se:

$$
\begin{gathered}
\vec{a}_{1}=\left[\sum_{k=1}^{n} \frac{\partial f_{k}(\eta)}{\partial \eta} \cdot r_{k}+\sum_{k=1}^{n} \frac{\partial f_{k}(\eta)}{\partial \eta} \cdot \frac{t_{k}}{2} \cdot \zeta \cdot v_{3 k}^{r}\right] \cdot \vec{i}_{r}(\theta)+ \\
{\left[\sum_{k=1}^{n} \frac{\partial f_{k}(\eta)}{\partial \eta} \cdot r_{k}+\sum_{k=1}^{n} \frac{\partial f_{k}(\eta)}{\partial \eta} \cdot \frac{t_{k}}{2} \cdot \zeta \cdot v_{3 k}^{z}\right] \cdot \vec{k}} \\
\vec{a}_{2}=\left[\sum_{k=1}^{n} f_{k}(\eta) \cdot \frac{t_{k}}{2} \cdot v_{3 k}^{r}\right] \cdot \vec{i}_{r}(\theta)+\left[\sum_{k=1}^{n} f_{k}(\eta) \cdot \frac{t_{k}}{2} \cdot v_{3 k}^{z}\right] \cdot \vec{k} \\
\vec{a}_{3}=\left[\sum_{k=1}^{n} f_{k}(\eta) \cdot r_{k}+\sum_{k=1}^{n} f_{k}(\eta) \cdot \frac{t_{k}}{2} \cdot \zeta \cdot v_{3 k}^{r}\right] \cdot \vec{i}_{\theta}
\end{gathered}
$$

A partir da base covariante pode-se determinar os versores normais e tangentes, que constituirão a base ortonormal, designada por $[Q]_{X}=\left[t: n: i_{\theta}\right]$, associada ao mesmo ponto. Sendo $\vec{t}$ o versor de $\vec{a}_{1}$ e $\vec{n}$ o resultado do produto externo de $\vec{i}_{\theta}$ com esse versor $(\vec{t})$ tem-se:

$$
\begin{gathered}
\vec{t}=\frac{\vec{a}_{1}}{\left|\vec{a}_{1}\right|} \\
\vec{n}=\vec{i}_{\theta} \times \vec{t}
\end{gathered}
$$

Fato importante, porém ainda não comentado até então, é a transformação do sistema de coordenadas natural $(\eta, \zeta, \theta)$ para o global da estrutura. Tal transformação é feita com auxílio da chamada matriz jacobiano, que é definida como $[J]=\left[F_{\zeta}\right]^{T}$. Considerando que cada um dos vetores da base co-variante representa uma coluna da matriz $\left[F_{\zeta}\right]$, tem-se:

$$
\left[F_{\zeta}\right]=\left[\begin{array}{ccc}
\frac{\partial r(\eta, \zeta)}{\partial \eta} & \frac{\partial r(\eta, \zeta)}{\partial \zeta} & 0 \\
\frac{\partial z(\eta, \zeta)}{\partial \eta} & \frac{\partial z(\eta, \zeta)}{\partial \zeta} & 0 \\
0 & 0 & r(\eta, \zeta)
\end{array}\right]
$$

De acordo com o referido, a matriz jacobiana tem a seguinte representação:

$$
[J]=\left[\begin{array}{ccc}
\frac{\partial r(\eta, \zeta)}{\partial \eta} & \frac{\partial z(\eta, \zeta)}{\partial \eta} & 0 \\
\frac{\partial r(\eta, \zeta)}{\partial \zeta} & \frac{\partial z(\eta, \zeta)}{\partial \zeta} & 0 \\
0 & 0 & r(\eta, \zeta)
\end{array}\right]
$$




\subsection{Modelo cinemático de Reissner-Mindlin}

O modelo cinemático de Reissner-Mindlin permite levar em conta deformações por cortante mantendo-se reta, entretanto, a normal à superfície média durante a deformação.

Para o caso em estudo, superfícies com axissimetria de forma e carregamento, os deslocamentos na direção do versor $i_{\theta}$ são nulos.

\subsubsection{Modelo de Reissner-Mindlin aplicado ao caso axissimétrico}

A equação representativa dos deslocamentos para um elemento, pode ser escrita em coordenadas cilíndricas, como segue

$$
\vec{u}(\eta, \zeta, \theta)=v(\eta, \zeta) \cdot \vec{i}_{r}(\theta)+w(\eta, \zeta) \cdot \vec{k}+u(\theta) \cdot \vec{i}_{\theta}
$$

A forma geral de, cada componente da equação 3.13, segundo os versores do sistema de referência está representada pelas expressões 3.14, já particularizadas para o caso axissimétrico:

$v(\eta, \zeta)=v_{o}(\eta)+z(\zeta) \cdot \varphi(\eta) \quad ; \quad w(\eta, \zeta)=w_{o}(\eta)+r(\zeta) \cdot \varphi(\eta) \quad$ e $\quad u(\theta)=0$

Nas primeiras duas equações da 3.14 as parcelas $v_{0}$ e $w_{0}$, correspondem às componentes do deslocamento, referido ao sistema global, de um ponto sobre a superfície média $\zeta=0$. As segundas parcelas daquelas mesmas equações referem-se ao efeito do giro sofrido pela normal. Como se ilustra na figura 19 o ângulo inicial entre os vetores $v_{2 k}$ e $v_{3 k}$ pode deixar de ser reto (passando para $\beta$ ), o que decorre da deformação relativa ao cisalhamento.

Para a descrição do vetor deslocamento de um ponto qualquer no interior do elemento, utiliza-se de procedimentos análogos aos feitos, e já descritos anteriormente, baseados na função de mapeamento. Inicialmente, determina-se o deslocamento de um ponto da normal atrelada a um nó do elemento e posteriormente utilizando as funções lagrangianas, mostradas no item 3.1.2, faz-se a interpolação para pontos internos situados em linhas de $\zeta=$ cte.

O vetor deslocamento de um ponto sobre a normal associada a um nó do elemento 


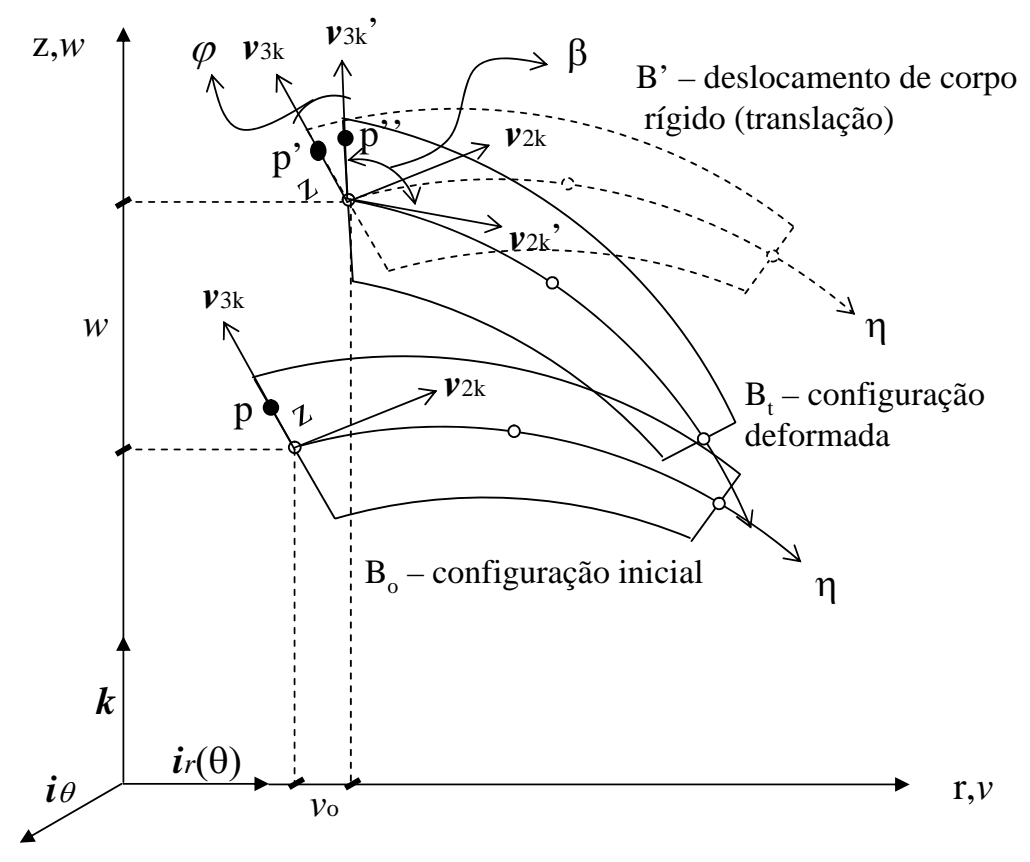

Figura 19: Modelo cinemático de primeira ordem

é obtido pela equação 3.15 , análoga à 3.4 :

$$
\vec{u}_{k}(\zeta, \theta)=\vec{u}_{o k}(\theta)+\frac{t_{k}}{2} \cdot \zeta \cdot \varphi_{k} \cdot \vec{v}_{2 k G}(\theta)
$$

onde o vetor $\vec{v}_{2 k}$ é referido à base global $\vec{i}_{r}(\theta)$ e $\vec{k}$.

Explicitando-se as componentes da equação 3.15, resulta:

$$
\vec{u}_{k}(\zeta, \theta)=\left\{\begin{array}{c}
v_{o k} \\
w_{o k}
\end{array}\right\}+\frac{t_{k}}{2} \cdot \zeta \cdot\left\{\begin{array}{c}
v_{2 k}^{r} \\
v_{2 k}^{z}
\end{array}\right\}
$$

Escrevendo, então o vetor $\vec{u}_{k}(\zeta, \theta)$ de acordo com suas componentes em relação à base global do sistema de coordenadas cilíndricas, obtém-se:

$$
\vec{u}_{k}(\zeta, \theta)=\left(v_{o k}+\frac{t_{k}}{2} \cdot \zeta \cdot \varphi_{k} \cdot v_{2 k}^{r}\right) \cdot \vec{i}_{r}(\theta)+\left(w_{k}+\frac{t_{k}}{2} \cdot \zeta \cdot \varphi_{k} \cdot v_{2 k}^{z}\right) \cdot \vec{k}
$$

Assim como feito para o vetor posição do elemento, a obtenção do vetor deslocamento para um ponto qualquer no seu interior emprega funções $f_{k}(\eta)$ de interpolação quadrática. Chega-se, assim, à forma compacta da equação que rege a cinemática do modelo: 


$$
\vec{u}_{k}(\eta, \zeta, \theta)=\sum_{k=1}^{n} f_{k}(\eta) \cdot \vec{u}_{k}(\zeta, \theta)
$$

ou reescrevendo-se de uma forma mais explícita, tem-se:

$$
\begin{array}{r}
\vec{u}_{k}(\eta, \zeta, \theta)=\left[\sum_{k=1}^{n} f_{k}(\eta) \cdot v_{o k}+\sum_{k=1}^{n} f_{k}(\eta) \cdot \frac{t_{k}}{2} \cdot \zeta \cdot \varphi_{k} \cdot v_{2 k}^{r}\right] \cdot \vec{i}_{r}(\theta)+ \\
{\left[\sum_{k=1}^{n} f_{k}(\eta) \cdot w_{k}+\sum_{k=1}^{n} f_{k}(\eta) \cdot \frac{t_{k}}{2} \cdot \zeta \cdot \varphi_{k} \cdot v_{2 k}^{z}\right] \cdot \vec{k}}
\end{array}
$$

\subsection{Equações constitutivas - Problema axissimétrico}

$\mathrm{Na}$ apresentação das equações constitutivas considera-se a base local $\left[t: n: i_{\theta}\right]$. As componentes gerais de tensão estão indicadas na figura 20. Considerando as hipóteses do modelo de Reissner - Mindlin e as restrições de axissimetria, as tensões normais $\sigma_{n n}$ são consideradas nulas e os deslocamentos na direção do versor $\vec{i}_{\theta}$ também são nulos. A condição de axissimetria implica ainda em tensões cisalhantes nulas na face perpendicular à direção $\vec{i}_{\theta}$.

Conseqüência disso é o valor nulo para as seguintes variáveis:

$$
\sigma_{n n}=\tau_{\theta n}=\tau_{\theta t}=0
$$

Na figura 20 observa-se o estado de tensões atuante num ponto genérico no elemento tendo-se em vista as considerações anteriores.

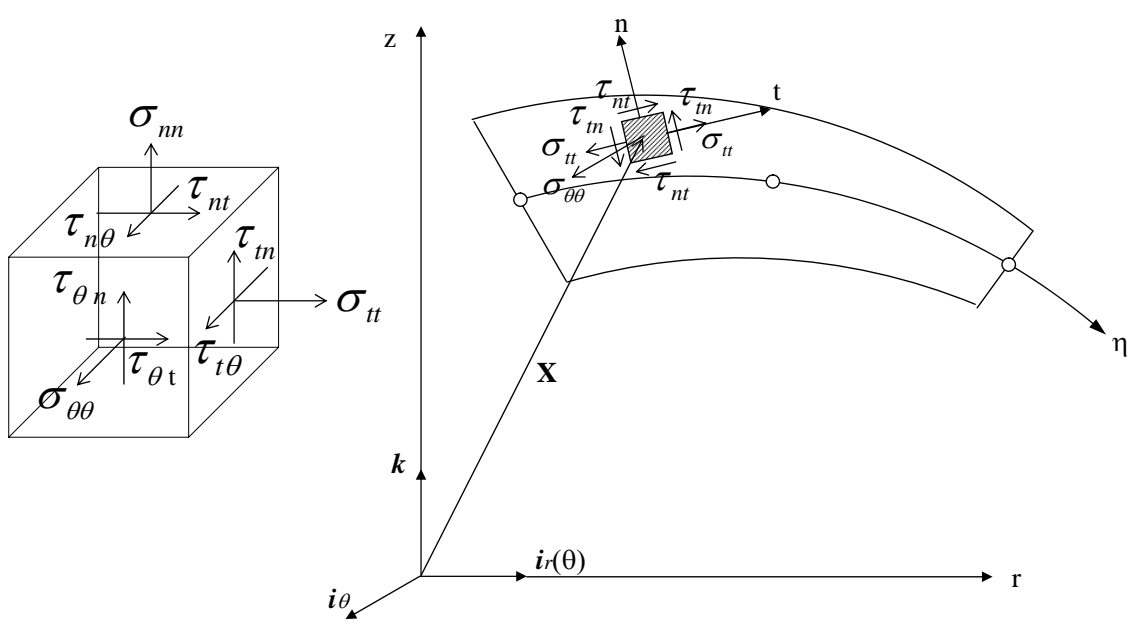

Figura 20: Estado de tensões para o problema axissimétrico 
Em princípio a relação constitutiva tridimensional para o caso geral (SORIANO, 2003) escreve-se na forma:

$$
\left\{\begin{array}{c}
\sigma_{\theta \theta} \\
\sigma_{t t} \\
\sigma_{n n} \\
\tau_{n t} \\
\tau_{n \theta} \\
\tau_{\theta t}
\end{array}\right\}=C_{E, \nu} \cdot\left[\begin{array}{cccccc}
1-\nu & \nu & \nu & 0 & 0 & 0 \\
\nu & 1-\nu & \nu & 0 & 0 & 0 \\
\nu & \nu & 1-\nu & 0 & 0 & 0 \\
0 & 0 & 0 & \frac{1-2 \cdot \nu}{2} & 0 & 0 \\
0 & 0 & 0 & 0 & \frac{1-2 \cdot \nu}{2} & 0 \\
0 & 0 & 0 & 0 & 0 & \frac{1-2 \cdot \nu}{2}
\end{array}\right] \cdot\left\{\begin{array}{c}
\varepsilon_{\theta \theta} \\
\varepsilon_{t t} \\
\varepsilon_{n n} \\
\gamma_{n t} \\
\gamma_{n \theta} \\
\gamma_{\theta t}
\end{array}\right\}
$$

onde a constante $C_{E, \nu}$ tem a seguinte expressão

$$
C_{E, \nu}=\frac{E}{(1+\nu) \cdot(1-2 \cdot \nu)}
$$

Levando-se em conta as restrições do modelo adotado da expressão 3.20 conclui-se que:

$$
\begin{gathered}
\sigma_{\theta \theta}=C_{E, \nu} \cdot\left[(1-\nu) \cdot \varepsilon_{\theta \theta}+\nu \cdot \varepsilon_{t t}+\nu \cdot \varepsilon_{n n}\right] \\
\sigma_{t t}=C_{E, \nu} \cdot\left[\nu \cdot \varepsilon_{\theta \theta}+(1-\nu) \cdot \varepsilon_{t t}+\nu \cdot \varepsilon_{n n}\right] \\
\sigma_{n n}=C_{E, \nu} \cdot\left[\nu \cdot \varepsilon_{\theta \theta}+\nu \cdot \varepsilon_{t t}+(1-\nu) \cdot \varepsilon_{n n}\right]=0 \\
\tau_{n t}=C_{E, \nu} \cdot \frac{(1-2 \cdot \nu)}{2} \cdot \gamma_{n t} \\
\tau_{n \theta}=C_{E, \nu} \cdot \frac{(1-2 \cdot \nu)}{2} \cdot \gamma_{n \theta}=0 \\
\tau_{t \theta}=C_{E, \nu} \cdot \frac{(1-2 \cdot \nu)}{2} \cdot \gamma_{\theta t}=0
\end{gathered}
$$

Das equações $3.23,3.25$ e 3.26 resultam as seguintes expressões para as deformações $\varepsilon_{n n}, \gamma_{n \theta}$ e $\gamma_{\theta t}$ : 


$$
\varepsilon_{n n}=\frac{-\nu}{(1-\nu)} \cdot\left(\varepsilon_{\theta \theta}+\varepsilon_{t t}\right) \quad ; \quad \gamma_{n \theta}=0 \quad \text { e } \quad \gamma_{\theta t}=0
$$

Substituindo-se a expressão da deformação $\varepsilon_{n n}$ nas equações 3.21 e 3.22, obtém-se para as tensões normais $\left(\sigma_{t t}\right)$ e $\left(\sigma_{\theta \theta}\right)$ as relações:

$$
\begin{aligned}
& \sigma_{t t}=C_{E, \nu} \cdot\left[\nu \cdot\left(\frac{1-2 \cdot \nu}{1-\nu}\right) \cdot \varepsilon_{\theta \theta}+\left(\frac{1-2 \cdot \nu}{1-\nu}\right) \cdot \varepsilon_{t t}\right] \\
& \sigma_{\theta \theta}=C_{E, \nu} \cdot\left[\left(\frac{1-2 \cdot \nu}{1-\nu}\right) \cdot \varepsilon_{\theta \theta}+\nu \cdot\left(\frac{1-2 \cdot \nu}{1-\nu}\right) \cdot \varepsilon_{t t}\right]
\end{aligned}
$$

Utilizando a notação matricial e aplicando o coeficiente de correção ao cisalhamento de Reissner, obtém-se, finalmente, a seguinte forma para a relação constitutiva do problema axissimétrico em questão

$$
\left\{\begin{array}{c}
\sigma_{\theta \theta} \\
\sigma_{t t} \\
\tau_{n t}
\end{array}\right\}=\frac{E}{1-\nu^{2}} \cdot\left[\begin{array}{ccc}
1 & \nu & 0 \\
\nu & 1 & 0 \\
0 & 0 & \frac{\kappa(1-\nu)}{2}
\end{array}\right] \cdot\left\{\begin{array}{c}
\varepsilon_{\theta \theta} \\
\varepsilon_{t t} \\
\gamma_{n t}
\end{array}\right\}
$$

Na equação 3.27, $\kappa=5 / 6$ é o coeficiente de correção ao cisalhamento de Reissner.

\subsection{Forma fraca do problema - Método de Galerkin}

Nesse item faz-se uma breve apresentação do equacionamento do problema axissimétrico de acordo com (SUAREZ; PROENÇA, 2005), a fim de obter a equação integral representativa da forma fraca do problema.

O problema em estudo insere-se na elasticidade linear, com restrições sobre os deslocamentos e as tensões em razão da axissimetria. Admitindo-se que $\sigma$ seja o tensor de tensões colocado em correspondência com um tensor de deformações compatíveis mediante o modelo constitutivo, as equações pertinentes a tal modelo são:

$$
\operatorname{div} \sigma=0 \mathrm{em} \Omega \quad ; \quad \sigma \vec{n}=\vec{p} \operatorname{em} \partial \Omega_{N} \quad ; \quad \vec{u}=\bar{u} \operatorname{em} \partial \Omega_{D}
$$

A primeira refere-se ao equilíbrio para um ponto no interior do volume, uma vez desconsideradas as forças de corpo. A segunda equação é do equilíbrio com forças por unidade de superfície aplicada na parte $\partial \Omega_{N}$ do contorno. A última relação exprime a 
condição de contorno em deslocamentos, na parte do contorno $\partial \Omega_{D}$. A figura 21 ilustra particularmente as parcelas $\partial \Omega_{N}$ e $\partial \Omega_{D}$ complementares do contorno.

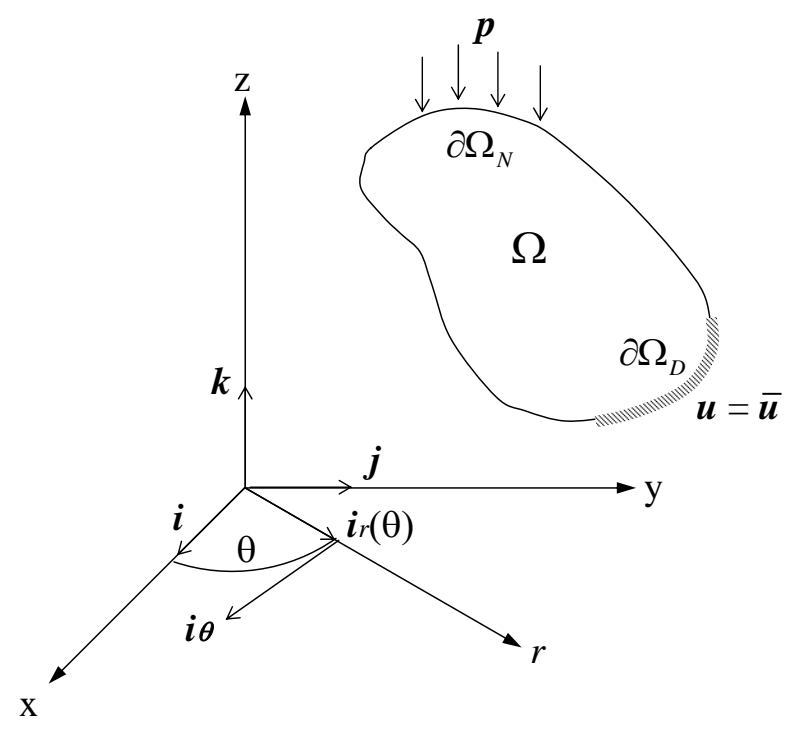

Figura 21: Esquema estático para modelagem do problema

O ponto de partida para a formulação fraca do problema é a condição de ortogonalidade entre o operador $L(\vec{u})$ e a função vetorial ponderadora $\phi$, homogênea em $\partial \Omega_{D}$ :

$$
\int_{\Omega} L(\vec{u}) \cdot \phi d \Omega=0
$$

onde $L(\vec{u})=\operatorname{div} \sigma(\vec{u})$ e $\vec{u}$ é um campo vetorial de deslocamentos cinematicamente admissíveis. Por simplificação, admite-se $\bar{u}=0$ em $\partial \Omega_{D}$

Tendo-se em vista o equilíbrio no domínio $\Omega$ a equação 3.28 fica:

$$
\int_{\Omega} \operatorname{div} \sigma(u) \cdot \phi d \Omega=0
$$

Considerando-se da álgebra tensorial que:

$$
\operatorname{div}(\sigma \phi)=\operatorname{div}(\sigma) \cdot \phi+\nabla \phi \cdot \sigma
$$

a equação 3.29 passa a:

$$
\int_{\Omega} \operatorname{div}[\sigma(u) \phi] d \Omega-\int_{\Omega} \sigma(u) \cdot \nabla \phi d \Omega=0
$$

Aplicando-se o teorema da divergência ao primeiro integrando da equação 3.30, 
obtém-se:

$$
\int_{\partial \Omega_{N}}[\sigma(u) \vec{n}] \cdot \phi d \Omega-\int_{\partial \Omega_{D}}[\sigma(u) \vec{n}] \cdot \phi d \Omega-\int_{\Omega} \sigma(u) \cdot \nabla \phi d \Omega=0
$$

Como $\phi$ deve ser nula em $\partial \Omega_{D}$ e $\sigma \cdot \vec{n}=\vec{p}$ em $\partial \Omega_{N}$, a equação 3.31 é reescrita da seguinte forma

$$
\int_{\partial \Omega_{N}} \vec{p} \cdot \phi \partial \Omega-\int_{\Omega} \sigma(u) \cdot \nabla \phi d \Omega=0
$$

Aplicando-se o método de Galerkin, que consiste em tomar como função ponderadora um campo vetorial de deslocamentos cinematicamente admissíveis e homogêneos em $\partial \Omega_{D}$, na equação 3.32 o gradiente da função ponderadora passa a representar um tensor "virtual" de deformações. Além disso, escrevendo-se o tensor das tensões em função da relação constitutiva (equação 3.27), valem as relações:

$$
\sigma(u)=C \varepsilon(u) \quad e \quad \nabla \phi=\varepsilon(\delta \vec{u})
$$

Obtém-se então, a expressão da forma fraca do problema

$$
\int_{\partial \Omega_{N}} \vec{p} \cdot \delta \vec{u} \partial \Omega-\int_{\Omega} C \varepsilon(u) \cdot \varepsilon(\delta \vec{u}) d \Omega=0
$$

$\mathrm{ou}$

$$
\int_{\Omega} \varepsilon^{T}(\delta u) C \varepsilon(\bar{u}) d \Omega=\int_{\partial \Omega_{N}} \vec{p} \cdot \delta \vec{u} \partial \Omega
$$

\subsection{Equações de Buhnov-Galerkin}

As equações de Buhnov-Galerkin constituem uma aproximação do campo de deslocamentos, construídas pela combinação de funções de forma definidas em sub-domínios do domínio do problema $\Omega$. As funções de forma $N_{i}(\eta)$ definidas, então, sobre os subdomínios $\omega_{i}$, estão vinculadas à rede de elementos finitos utilizada para discretizar a estrutura. A figura 22 ilustra esse conceito em campo unidimensional.

Dessa forma, pode-se escrever a função representativa da aproximação dos deslocamentos para o caso bidimensional como: 


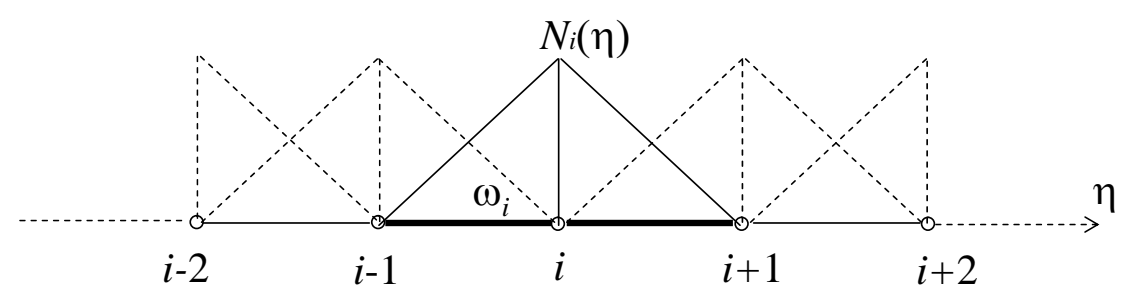

Figura 22: Sub-domínios $\omega_{i}$ e funções de forma $N_{i}(\eta)$

$$
\vec{u}=\vec{u}_{h}(\eta, \zeta)
$$

Utilizando a descrição do elemento, mostrada no item 3.2.1, e considerando-se três graus de liberdade por nó, a função de aproximação dos deslocamentos se escreve como segue:

$$
\vec{u}_{h}(\eta, \zeta)=\left\{\begin{array}{c}
v \\
w
\end{array}\right\}=\left[\begin{array}{ccccc}
\ldots & N_{i}(\eta) & 0 & N_{i}(\eta) \cdot \frac{t_{i}}{2} \cdot \zeta \cdot v_{2 i}^{r} & \ldots \\
\ldots & 0 & N_{i}(\eta) & N_{i}(\eta) \cdot \frac{t_{i}}{2} \cdot \zeta \cdot v_{2 i}^{z} & \ldots
\end{array}\right] \cdot\left\{\begin{array}{c}
\vdots \\
v_{i} \\
w_{i} \\
\varphi_{i} \\
\vdots
\end{array}\right\}
$$

com $i=1, \ldots, N_{\text {nuv }}$, onde $N_{\text {nuv }}$ é o número de nuvens da rede de elementos finitos.

Reescrevendo-se a equação 3.34 em notação compacta, tem-se

$$
\vec{u}_{h}(\eta, \zeta)=N U
$$

\subsubsection{Deformações - Sistema local de coordenadas}

É sabido que o tensor das deformações é obtido através do gradiente do tensor dos deslocamentos. Aplicando o operador gradiente em relação às coordenadas naturais à expressão 3.13 observando que $\frac{\partial \vec{i}_{r}(\theta)}{\partial \theta}=\vec{i}_{\theta}$ e escrevendo-o na forma vetorial, obtém-se:

$$
\nabla_{\zeta} \vec{u}^{T}(\eta, \zeta)=\left\{\begin{array}{lllllllll}
\frac{\partial v}{\partial \eta} & \frac{\partial w}{\partial \zeta} & 0 & \frac{\partial v}{\partial \eta} & \frac{\partial w}{\partial \zeta} & 0 & 0 & 0 & v
\end{array}\right\}
$$

Com aplicação do operador gradiente à equação 3.35, restrita ao elemento, ela 
passa a ter a seguinte forma:

$$
\nabla_{\zeta} \vec{u}(\eta, \zeta)=\partial_{\zeta} N U
$$

Explicitando a equação 3.37, com a utilização das expressões 3.36 e 3.34, o gradiente dos deslocamentos fica caracterizado como segue:

$$
\nabla_{\zeta} \vec{u}(\eta, \zeta)=\left[\begin{array}{ccccc}
\ldots & \frac{\partial N_{i}(\eta)}{\partial \eta} & 0 & \frac{\partial N_{i}(\eta)}{\partial \eta} \cdot \frac{t_{i}}{2} \cdot \zeta \cdot v_{2 i}^{r} & \ldots \\
\ldots & 0 & 0 & \frac{\partial N_{i}(\eta)}{\partial \eta} \cdot \frac{t_{i}}{2} \cdot v_{2 i}^{r} & \ldots \\
\ldots & 0 & 0 & 0 & \ldots \\
\ldots & 0 & \frac{\partial N_{i}(\eta)}{\partial \eta} & \frac{\partial N_{i}(\eta)}{\partial \eta} \cdot \frac{t_{i}}{2} \cdot \zeta \cdot v_{2 i}^{z} & \ldots \\
\ldots & 0 & 0 & \frac{\partial N_{i}(\eta)}{\partial \eta} \cdot \frac{t_{i}}{2} \cdot v_{2 i}^{z} & \ldots \\
\ldots & 0 & 0 & 0 & \ldots \\
\ldots & 0 & 0 & 0 & \ldots \\
\ldots & 0 & 0 & 0 & \ldots \\
\ldots & N_{i}(\eta) & 0 & N_{i}(\eta) \cdot \frac{t_{i}}{2} \cdot \zeta \cdot v_{2 i}^{r} & \ldots
\end{array}\right] \cdot\left\{\begin{array}{c}
\vdots \\
v_{i} \\
w_{i} \\
\varphi_{i} \\
\vdots
\end{array}\right\}
$$

A equação 3.38 fornece o gradiente de deslocamentos com referência ao sistema de coordenadas naturais do elemento $(i=1,2,3)$. Pode-se passar a uma representação em relação ao sistema global de coordenadas, com a aplicação do operador jacobiano $(\mathbf{J})$, que é definido como

$$
\mathbf{J}=\left[\begin{array}{ccc}
J^{-1} & 0 & 0 \\
0 & J^{-1} & 0 \\
0 & 0 & J^{-1}
\end{array}\right]
$$

Sendo $J$, a matriz jacobiana, definida na equação 3.12, do item 3.1.3.

Logo, o gradiente dos deslocamentos, relativo ao sistema global de coordenadas se escreve

$$
\nabla_{r} \vec{u}(r, z)=\mathbf{J} \nabla_{\zeta} \vec{u}(\eta, \zeta)
$$

Cabe, agora, relacionar os sistemas local e global, visto que as equações constitutivas, foram deduzidas no sistema local do elemento, e as incógnitas a serem determinadas referidas ao sistema global da estrutura. 
O operador utilizado para transformação de coordenadas é uma matriz de rotação, no caso aqui, associada a cada ponto e sua base ortonormal $\left[\vec{t}: \vec{n}: \vec{i}_{\theta}\right]$.

$$
\mathbf{R}=\left[\begin{array}{ccc}
t_{r} \cdot[Q]_{X}^{T} & t_{z} \cdot[Q]_{X}^{T} & 0 \\
n_{r} \cdot[Q]_{X}^{T} & n_{z} \cdot[Q]_{X}^{T} & 0 \\
0 & 0 & {[Q]^{T}}
\end{array}\right]
$$

Assim, escrevendo a equação 3.39 em função de 3.37 e associando a ela o operador de rotação $(\mathbf{R})$, o gradiente de deslocamentos, relativo ao sistemas local do elemento, se escreve

$$
\nabla_{t} \vec{u}(\eta, \zeta)=\mathbf{R} \cdot \mathbf{J} \cdot \partial_{\zeta} N \cdot U
$$

Lembrando que o gradiente dos deslocamentos representa o tensor das deformações, pode-se escrevê-lo da seguinte maneira:

$$
\varepsilon_{t}=\mathbf{H} \cdot \mathbf{R} \cdot \mathbf{J} \cdot \partial_{\zeta} N \cdot U
$$

Aqui, o operador $\mathbf{H}$ representa uma matriz auxiliar, utilizada para se obter diretamente o tensor das deformações a partir do gradiente $\nabla_{t} \vec{u}(\eta, \zeta)$, e tem a seguinte configuração:

$$
\mathbf{H}=\left[\begin{array}{lllllllll}
1 & 0 & 0 & 0 & 0 & 0 & 0 & 0 & 0 \\
0 & 1 & 1 & 0 & 0 & 0 & 0 & 0 & 0 \\
0 & 0 & 0 & 0 & 0 & 0 & 0 & 0 & 1
\end{array}\right]
$$

Com o intuito de simplificar as expressões posteriores, escreve-se o tensor das deformações em função da matriz de deformações $\left(B_{t}\right)$, sendo ela

$$
B_{t}=\mathbf{H} \cdot \mathbf{R} \cdot \mathbf{J} \cdot \partial_{\zeta} N
$$

Portanto, o tensor das deformações tem para expressão final:

$$
\varepsilon_{t}=B_{t} \cdot U
$$




\subsubsection{Matriz de Rigidez e Vetor de Forças nodais equivalentes}

Com o exposto até então é possível determinar a Matriz de Rigidez e o Vetor de Forças nodais equivalentes. Consideram-se em particular a expressão da forma fraca do problema (equação 3.33) e as equações 3.32 e 3.42. Fazendo as devidas substituições, tem-se a seguinte equação:

$$
\sum_{e=1}^{n}\left(\int_{\Omega_{e}} B_{t}^{T} C B_{t} d \Omega\right) U \delta U=\left(\int_{\partial \Omega_{N}} N^{T} \vec{p} d \partial \Omega\right) \delta U
$$

O vetor $\delta U$ representa o vetor de deslocamentos ditos virtuais, $\Omega_{e}$ o domínio do elemento, $n$ o número de elementos do problema.

Impondo que a equação 3.43 seja válida para todo $\delta U$ compatível e homogênea em $\partial \Omega_{D}$ obtém-se a forma conhecida do MEF:

$$
\sum_{e=1}^{n}\left(\int_{\Omega_{e}} B_{t}^{T} C B_{t} d \Omega\right) U=\int_{\partial \Omega_{N}} N^{T} \vec{p} d \partial \Omega
$$

Definindo:

$$
\begin{aligned}
& K_{G}=\sum_{e=1}^{n} \int_{\Omega_{e}} B_{t}^{T} C B_{t} d \Omega \\
& F_{G}=\sum_{e=1}^{n} \int_{\partial \Omega_{N}} N^{T} \vec{p} d \partial \Omega
\end{aligned}
$$

a equação 3.44 pode ser reescrita na forma da equação matricial

$$
K_{G} U=F_{G}
$$

onde $K_{G}$ é a matriz de rigidez da estrutura e $F_{G}$ é o vetor de forças nodais equivalentes do problema.

A formulação apresentada neste capítulo diz respeito ao MEF convencional; resta agora introduzir as funções de enriquecimento,a fim de se obter a formulação do MEFG (Método dos Elementos Finitos Generalizados). 


\section{Método dos Elementos Finitos Generalizados}

Como visto no capítulo 2, mesmo com o recurso das simplificações conceituais, o modelo matemático das cascas de revolução com carregamento axissimétrico requer a resolução de um sistema de equações diferenciais em derivadas parciais.

Para se obter a solução para problemas mais gerais envolvendo cascas com diferentes tipos de carregamento e vinculação, por exemplo, é necessário o uso de ferramentas numéricas para o cálculo aproximado dos esforços e deslocamentos dessas estruturas. Nesse sentido se aplica, com grande aptidão, o Método dos Elementos Finitos (MEF). Entretanto, há limitações na forma convencional do MEF, particularmente devido ao elevado custo computacional quando redes muito refinadas de elementos são exigidas.

No intuito de aprimorar as ferramentas para resolução dos problemas das cascas, optou-se pelo uso de uma forma não-convencional do MEF. Tal forma é denominada Método dos Elementos Finitos Generalizados (MEFG).

Segundo (BARROS, 2002), o MEFG incorpora recursos dos chamados Métodos Sem Malha ao MEF convencional. São chamados de Métodos Sem Malha aqueles nos quais o conjunto de equações que governam o problema não depende da malha no sentido forte, isso quer dizer que a malha não precisa satisfazer os requisitos de conformidade exigidos no MEF convencional (SUAREZ, 2003). Entre o Método Sem Malha e o MEFG destaca-se uma diferença básica: a escolha da partição da unidade (PU). No MEFG a PU é fornecida pela funções de forma do MEF convencional (tais que localmente apresentam soma unitária) e nos Métodos Sem Malha a PU é obtida com a utilização do Método dos Mínimos Quadrados Móveis, (NIRSCHL, 2005)

O MEFG caracteriza-se também pela possibilidade de multiplicação da PU por uma função chamada de "enriquecedora"introduzindo novos parâmetros associados aos nós da rede de elementos. Normalmente os parâmetros nodais básicos são deslocamentos e rotações. Com o enriquecimento imposto pelo MEFG, os novos parâmetros nodais, podem não ter significado físico, porém também podem ser interpretados como graus de 
liberdade.

Uma prática de enriquecimento da solução é o chamado refino p, onde ao invés de se aumentar o número de elementos da rede (refino h), procura-se melhorar a função de aproximação utilizando-se uma menor quantidade de elementos finitos.

Neste trabalho, a forma de enriquecimento utilizada é em essência um refino p, pois as funções de forma que compõem a PU são multiplicadas por funções polinomiais enriquecedoras, levando a polinômios de maior ordem e propiciando uma melhor aproximação da solução.

Apesar do enriquecimento ter característica local, podendo ser seletivo, isto é, sobre um número arbitrário de nós, a PU garante a continuidade da aproximação global.

\subsection{Enriquecimento da partição da unidade}

No MEFG as funções de aproximação são enriquecidas mediante a multiplicação da PU por funções capazes de levar a função produto a melhores resultados em relação a solução.

O enriquecimento é feito sobre o subdomínio $\left(\omega_{\alpha}\right)$ da PU, ver figura 23, englobando os dois elementos contíguos a um nó comum. Aos nós possíveis de serem atreladas funções de enriquecimento será dado o nome de nó ativo. Essa distinção visa diferenciar os nós do elemento visto que dos seus três nós apenas os de extremidades poderão ser enriquecidos.

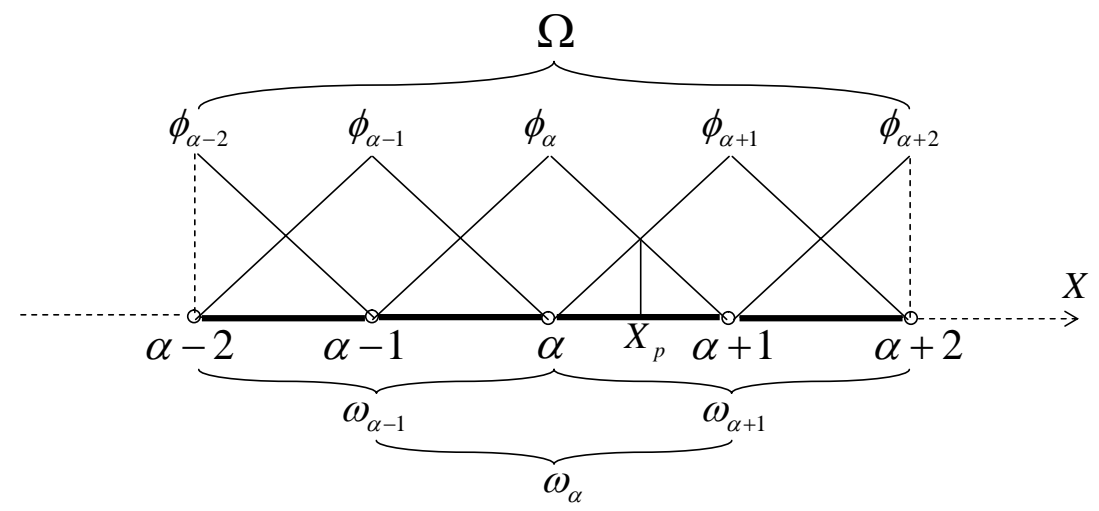

Figura 23: Partição da unidade e subdomínio $\omega_{\alpha}$

Apenas a título de compreensão do citado acima, observa-se na figura $24 \mathrm{um}$ conjunto composto de dois elementos $\left(E_{1}\right.$ e $\left.E_{2}\right)$ num domínio $(\Omega)$ e com três nós ativos $\left(\alpha_{1}, \alpha_{3}\right.$ e $\left.\alpha_{5}\right)$. 


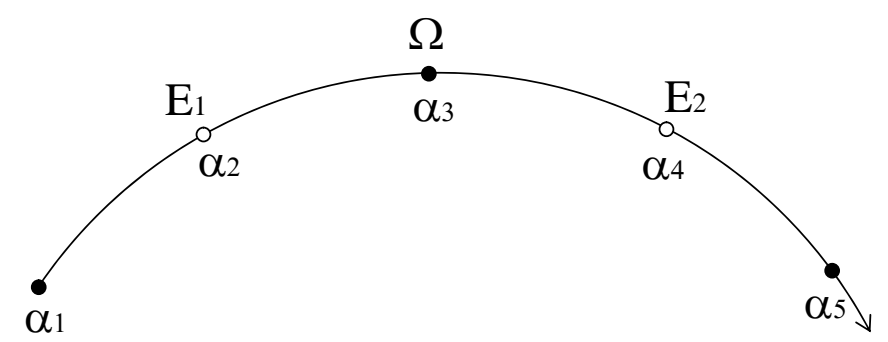

Figura 24: Nós ativos do elemento

\subsection{Espaço de aproximação}

Como citado na seção 4.1, as funções de aproximação do MEFG são construídas sobre subdomínios $\left(\omega_{\alpha}\right)$, que de agora em diante serão denominados nuvens.

As funções de enriquecimento utilizadas nesse trabalho são do tipo polinomial, pois apresentam boa regularidade e capacidade de aproximação. Como forma de representação do espaço de tais funções utiliza-se a notação $\left(V_{\alpha}^{p}\right)$, onde $p$ significa o grau do polinômio de maior ordem utilizado no enriquecimento e $\alpha$ a nuvem à qual a base está associada.

De uma maneira mais formal é possível descrever o espaço local das funções de aproximação como:

$$
V_{\alpha}^{p}=L_{i, \alpha}(\bar{x}) \quad \text { com } \quad 0 \leq i \leq p
$$

onde $L_{i, \alpha}($.$) é o conjunto formado por monômios de uma base polinomial de grau i$ associada à nuvem $\alpha$.

Sendo assim o conjunto de funções de aproximação enriquecidas tem a seguinte configuração:

$$
F_{\alpha}^{p}=\left\{\left\{\phi_{\alpha} \cdot V_{\alpha}^{p}\right\}: 1 \leq \alpha \leq N_{\omega}\right\}
$$

Na equação $4.1 \phi_{\alpha}$ é a partição da unidade e $N_{\omega}$ é o número de nuvens que cobrem o domínio $\Omega$.

A construção das funções de aproximação emprega um sistema local de referência associado às nuvens $\left(\omega_{\alpha}\right)$, ou seja, tem sua origem de coordenadas no nó base da nuvem $\alpha$, como mostrado na figura 25 


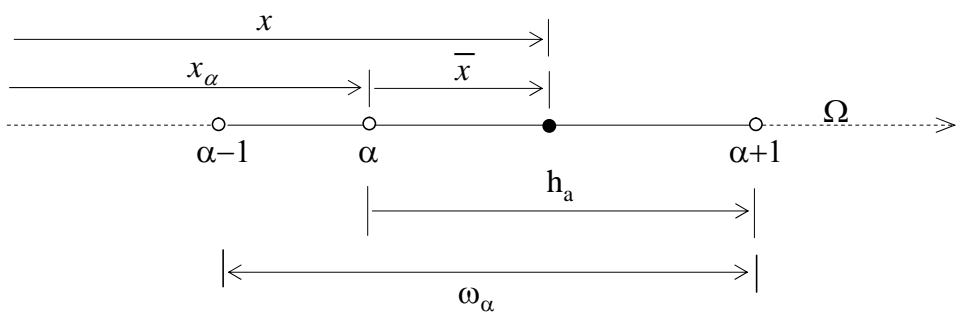

Figura 25: Sistema de referência associado à nuvem $\alpha$

Dessa forma, um ponto qualquer no interior da nuvem terá sua abscissa dada por:

$$
\bar{x}=x-x_{\alpha}
$$

onde $x$ é a abscissa referida ao sistema global de coordenadas.

Uma forma ainda mais conveniente é a de se utilizar as abscissas normalizadas em relação à variável $h_{\alpha}$, aqui definida como raio da nuvem:

$$
\xi=\frac{x-x_{\alpha}}{h_{\alpha}}=\frac{\bar{x}}{h_{\alpha}}
$$

A normalização sugerida implica que as abscissas relativas às nuvens estejam contidas no seguinte conjunto $\omega_{\alpha}=\{\xi \in R:|\xi| \leq 1\}$.

Apenas com a intenção de ilustrar os espaços de aproximação, segue na figura 26, o exemplo de um conjunto $F_{\alpha}^{p} \operatorname{com} p=4$ e $\alpha=1$, associado a uma nuvem com centro em $\xi=0$.

Um fato inconveniente que pode surgir quando se utiliza de funções polinomiais, tanto para PU como para as funções de enriquecimento, é que o conjunto $F_{\alpha}^{p}$ pode ter elementos linearmente dependentes, gerando assim uma matriz de rigidez do tipo positiva semi - definida.

Tal inconveniente pode ser contornado com a escolha adequada das funções de enriquecimento ou de modo mais geral empregando-se um algoritmo especial para resolução do sistema de equações representativo da estrutura. Tal algoritmo será apresentado no capitulo 5 desta dissertação. 
Partição da Unidade

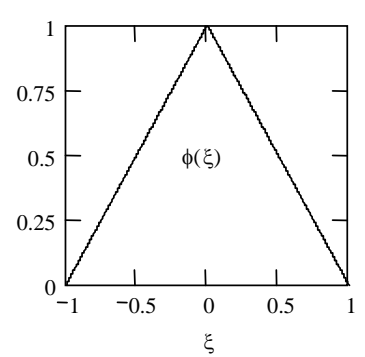

$$
\begin{aligned}
& \phi(\xi)=\left\{\begin{array}{l}
\xi+1 \Rightarrow-1 \leq \xi \leq 0 \\
-\xi+1 \Rightarrow 0 \leq \xi \leq 1
\end{array}\right. \\
& V_{1}^{4}=\left\{1, \xi, \xi^{2}, \xi^{3}, \xi^{4}\right\}
\end{aligned}
$$

Funções enriquecidas
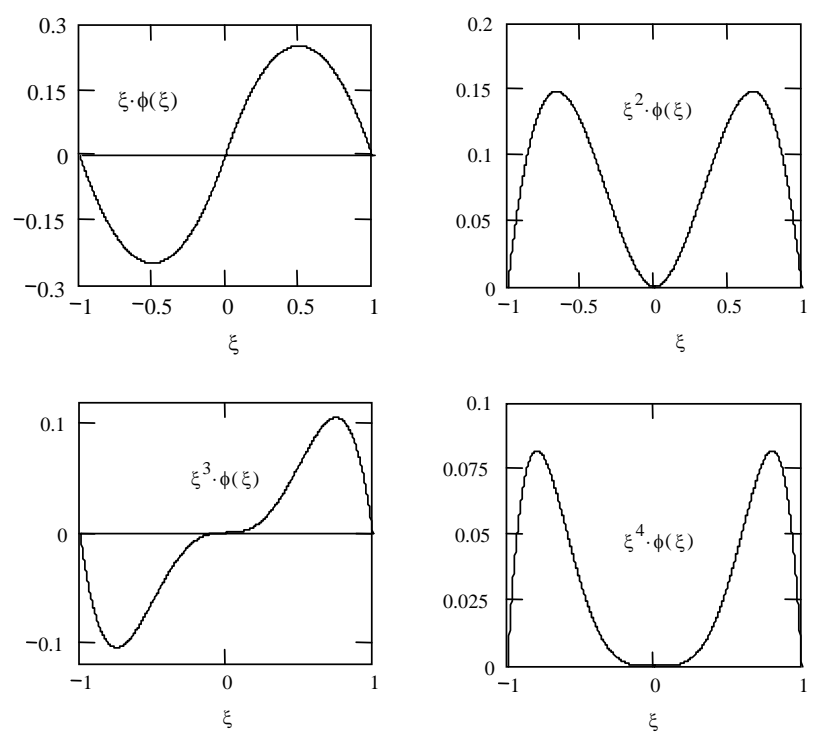

Figura 26: Partição da unidade e funções enriquecidas

\subsection{Espaço de aproximação - Domínio curvo}

A técnica desenvolvida aqui constrói o espaço de aproximação sobre o domínio paramétrico global do problema. A sistemática utilizada para parametrização do domínio propicia para alguns casos a possibilidade de se representar com exatidão a geometria da estrutura, quando conhecida a equação da curva representativa da superfície, ou com grande precisão, uma curva qualquer quando se conhecem apenas as coordenadas de alguns de seus pontos.

\subsubsection{Parametrização do domínio global}

Nesta seção é mostrada a forma de parametrização global do problema. Parte-se de um domínio adimensional local do elemento, onde estão definidas as funções de forma em coordenadas naturais $\eta$, e, mediante um mapeamento regular, define-se a coordenada dimensional paramétrica $s$.

Agora, com o domínio global $(\Sigma)$ conhecido, pelo uso de um segundo mapeamento $C_{1}(s)$, constrói-se a relação entre a coordenada paramétrica e as coordenadas de pontos de $\Sigma$ aos quais se associam vetores posição $\vec{X}(r, z, \theta)$. A figura 27 ilustra essa seqüência.

A função de mapeamento $C(\eta)$ que relaciona as coordenadas do domínio natural do problema $(-1 \leq \eta \leq+1)$ com o sistema paramétrico global $\left(s_{i-1} \leq s \leq s_{i+1}\right)$ emprega 


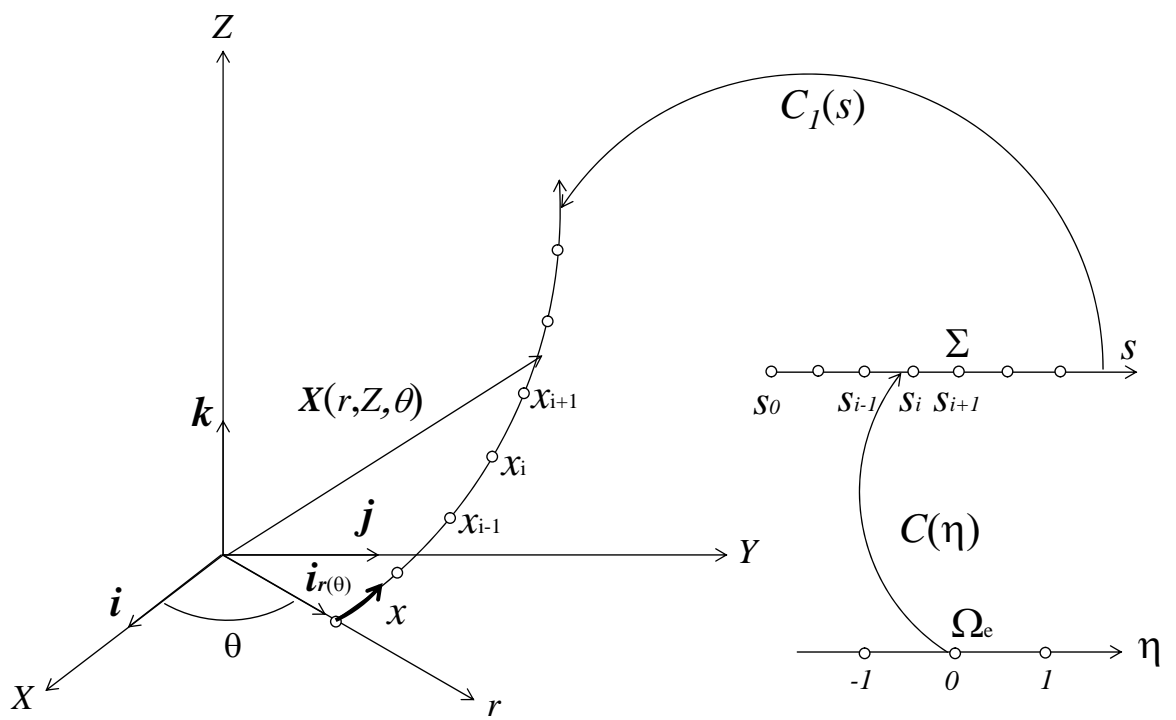

Figura 27: Parametrização do sistema de coordenadas

as mesmas funções lagrangianas citadas no item 3.1.2.

Dessa forma, por exemplo, pode-se escrever as coordenadas do domínio paramétrico $\Sigma$ como:

$$
s=\sum_{i=1}^{n} g_{i}(\eta) \cdot s_{i}
$$

O segundo mapeamento leva pontos do domínio paramétrico para o domínio físico. Em termos gerais um vetor posição no domínio físico escreve-se como:

$$
\vec{X}(r, z, \theta)=r(s) \cdot \vec{i}_{r}(\theta)+z(s) \cdot \vec{k}
$$

sendo $r(s)$ e $z(s)$ componentes obtidas por mapeamento a partir de $\Sigma$ genericamente representado por $r(s)=C_{r}(s)$ e $z(s)=C_{z}(s)$. Se a curva do domínio físico tem descrição exata o mapeamento opera-se diretamente.

A título de ilustração do exposto anteriormente, considera-se exemplo do mapeamento de um arco de circunferência. Para tal situação sabe-se que o domínio paramétrico pode ser descrito como $s \in \Sigma=\{0 \leq s \leq \pi / 2\}$.

As componentes do domínio físico resultam:

$$
r(s)=R_{c} \cdot \cos (s) \quad \text { e } \quad z(s)=R_{c} \cdot \operatorname{sen}(s)
$$

Vale lembrar que $R_{c}$ é o raio do arco de circunferência descrita. 
Substituindo-se a equação 4.2 nas expressões de $r(s)$ e $z(s)$, combinam-se os dois mapeamentos na forma:

$$
\begin{aligned}
& r(s)=R_{c} \cdot \cos \left(\sum_{i=1}^{n} g_{i}(\eta) \cdot s_{i}\right) \\
& z(s)=R_{c} \cdot \operatorname{sen}\left(\sum_{i=1}^{n} g_{i}(\eta) \cdot s_{i}\right)
\end{aligned}
$$

Se a curva no domínio físico não tem descrição exata ela pode ser descrita aproximadamente com a ajuda das funções de interpolação lagrangianas. Em primeiro lugar, determinam-se componentes $r_{i}$ e $z_{i}$ de pontos base do domínio físico por:

$$
r_{i}=C_{r}\left(s_{i}\right) \quad e \quad z_{i}=C_{z}\left(s_{i}\right)
$$

A interpolação de pontos sobre a curva no plano $(r, z)$ é então feita utilizando as funções lagrangianas. O vetor posição $\vec{X}(\eta, \theta)$ pode ser escrito como:

$$
\vec{X}(\eta, \theta)=\left[\sum_{i=1}^{n} g_{i}(\eta) \cdot r_{i}\right] \cdot \vec{i}_{r}(\theta)+\left[\sum_{i=1}^{n} g_{i}(\eta) \cdot z_{i}\right] \cdot \vec{k}
$$

Em resumo, a seqüência de mapeamentos comentada aplica-se para qualquer curva, seja ela de equação conhecida ou não. Para relações conhecidas entre as variáveis proporciona-se a descrição exata. Para curvas desconhecidas, onde não se tem uma relação definida entre as variáveis, a aproximação da curva no plano $(r, z)$ se dará por trechos interpolados de acordo com a função lagrangiana escolhida para tal.

No espaço paramétrico, por conveniência uma transformação de coordenadas é feita, como na seção 4.2, para o sistema local da nuvem e com normalização das abscissas locais em relação ao raio $h_{\alpha}$ da nuvem (maior distancia medida do nó central aos extremos da nuvem):

$$
\xi=\frac{s-s_{\alpha}}{h_{\alpha}}
$$

Adota-se, então, uma base de enriquecimento associada à nuvem $\alpha$. Tal base e seu gradiente são representados como:

$$
\left\{L_{i}(\xi)\right\}_{\alpha}=\left\{1, \xi, \ldots, \xi^{p}\right\} \quad \text { e } \quad\left\{\frac{d L_{i}(\xi)}{d \xi}\right\}_{\alpha}=\left\{0,1, \ldots, p \cdot \xi^{p-1}\right\}
$$

A obtenção das funções enriquecidas é feita pelo produto da PU pela base de funções de enriquecimento. Assim sendo, e de acordo com a equação 4.1, tem-se: 


$$
F_{\alpha}^{p}=\left\{\psi_{i}(\eta)\right\}_{\alpha}=\left\{\phi_{\alpha}(\eta), \phi_{\alpha}(\eta) \cdot \xi, \ldots, \phi_{\alpha}(\eta) \cdot \xi^{p}\right\}
$$

As funções de forma $\phi_{\alpha}(\eta)$ que compõem a PU são definidas pelas mesmas funções lagrangianas já mostradas anteriormente em 3.1.2; isto é:

(a)

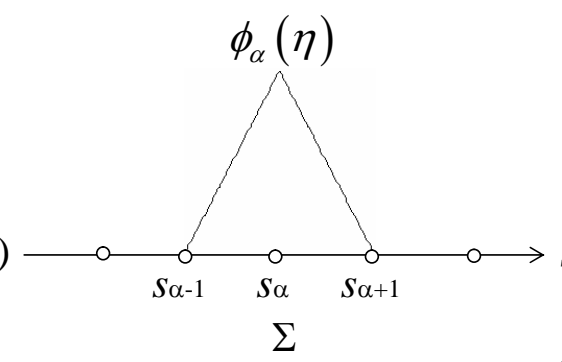

(b)
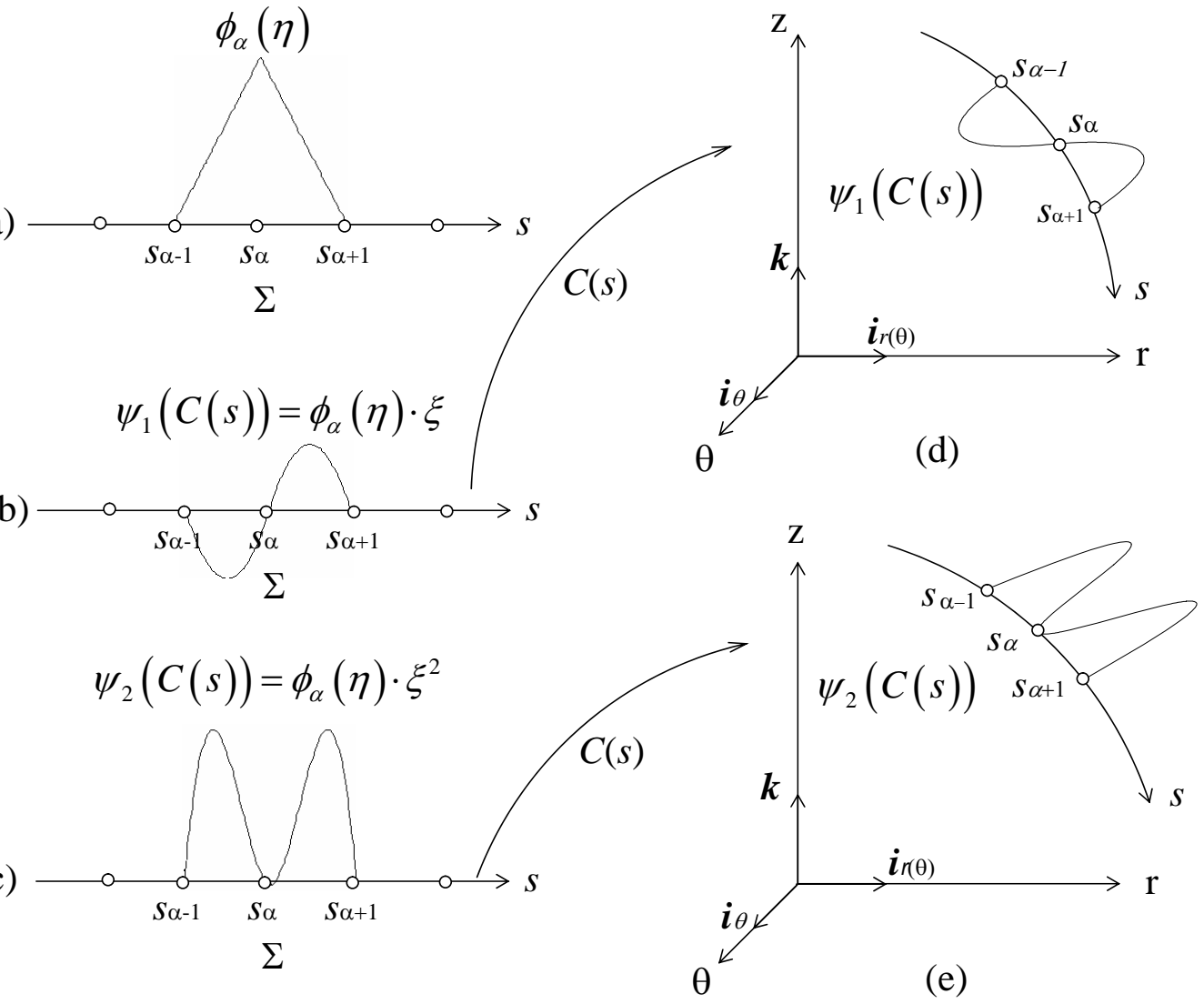

Figura 28: Funções de aproximação enriquecidas

$$
\phi_{\alpha}(\eta)=\left\{\begin{array}{lll}
g_{1}(\eta)=\frac{1}{2} \cdot(1-\eta) & \text { para } & s_{\alpha-1} \leq s \leq s_{\alpha} \\
g_{2}(\eta)=\frac{1}{2} \cdot(1+\eta) & \text { para } & s_{\alpha} \leq s \leq s_{\alpha+1}
\end{array}\right.
$$

A figura 28 ilustra um conjunto de funções enriquecidas.

Nas operações futuras de montagem da matriz de rigidez e vetor de forças nodais equivalentes, faz-se necessário o conhecimento do gradiente do espaço das funções de enriquecimento, em relação à coordenada do domínio natural do elemento $(\eta)$.

Utilizando a regra da cadeia para derivação implícita, tem-se:

$$
\frac{d L_{i}}{d \eta}=\frac{d L_{i}}{d \xi} \cdot \frac{d \xi}{d s} \cdot \frac{d s}{d \eta}
$$


Da derivação da equação 4.5 vem:

$$
\frac{d \xi}{d s}=\frac{1}{h_{\alpha}}
$$

Com isso, o gradiente do espaço enriquecido assume a seguinte forma:

$$
\frac{d L_{i}}{d \eta}=\frac{1}{h_{\alpha}} \cdot \frac{d L_{i}}{d \xi} \cdot \frac{d s}{d \eta}
$$

\subsubsection{Deslocamentos com o uso do espaço de aproximação enriquecido}

Como a determinação do espaço da aproximação vem do produto da PU pelas funções de enriquecimento do espaço $V_{\alpha}^{p}$, o vetor dos deslocamentos $\vec{u}(\eta, \zeta, \theta)$, levando-se em consideração as nuvens que contém o ponto, fica expresso, de acordo com as equações 3.19 e 4.6, da seguinte forma:

$$
\begin{aligned}
\vec{u}(\eta, \zeta, \theta)=\{ & \left.\sum_{j=1}^{n}\left[\sum_{k=1}^{m}\left(\psi_{k}^{j}(\eta) \cdot v_{o k}^{j}\right)+\sum_{k=1}^{m}\left(\psi_{k}^{j}(\eta) \cdot \frac{t_{j}}{2} \cdot \zeta \cdot \varphi_{k}^{j} \cdot v_{2 j}^{r}\right)\right]\right\} \cdot \vec{i}_{r}(\theta)+ \\
& \left\{\sum_{j=1}^{n}\left[\sum_{k=1}^{m}\left(\psi_{k}^{j}(\eta) \cdot w_{o k}^{j}\right)+\sum_{k=1}^{m}\left(\psi_{k}^{j}(\eta) \cdot \frac{t_{j}}{2} \cdot \zeta \cdot \varphi_{k}^{j} \cdot v_{2 j}^{z}\right)\right]\right\} \cdot \vec{k}
\end{aligned}
$$

onde $n$ é número de nós ativos ou de nuvens que contém o ponto considerado (para o caso unidimensional, sempre duas nuvens cobrirão o ponto); $m$ é o número de funções de enriquecimento. Os termos $v_{o k}^{j}, w_{o k}^{j}$ e $\varphi_{k}^{j}$ são parâmetros nodais introduzidos, devido a aplicação das funções de enriquecimento do espaço $V_{\alpha}^{p}$.

\subsection{Construção da matriz de rigidez e vetor de forças nodais equi- valentes - Base enriquecida}

A montagem da matriz de rigidez e do vetor de forças nodais equivalentes, considerando-se as funções enriquecidas, segue os mesmos passos dos itens 3.5.1 e 3.5.2.

Seguindo, portanto, o mesmo processo descrito nos itens 3.5.1 e 3.5.2, parte-se da expressão do gradiente dos deslocamentos:

$$
\nabla \vec{u}_{\zeta}=\partial_{\zeta} N \cdot U
$$


As variáveis $N$ e $U$, são afetadas pelo enriquecimento das funções de aproximação. A aplicação de tais funções, transforma as parcelas da equação 3.34 para:

$$
\begin{gathered}
N=\left[\begin{array}{cccccccccc}
\ldots & \psi_{1}^{j}(\eta) & 0 & \psi_{1}^{j}(\eta) \cdot \frac{t_{j}}{2} \cdot \zeta \cdot v_{2 j}^{r} & \ldots & \psi_{m}^{j}(\eta) & 0 & \psi_{m}^{j}(\eta) \cdot \frac{t_{j}}{2} \cdot \zeta \cdot v_{2 j}^{r} & \ldots \\
\ldots & 0 & \psi_{1}^{j}(\eta) & \psi_{1}^{j}(\eta) \cdot \frac{t_{j}}{2} \cdot \zeta \cdot v_{2 j}^{r} & \ldots & 0 & \psi_{m}^{j}(\eta) & \psi_{m}^{j}(\eta) \cdot \frac{t_{j}}{2} \cdot \zeta \cdot v_{2 j}^{r} & \ldots
\end{array}\right] \\
U^{T}=\left\{\begin{array}{llllllllll}
\ldots & v_{o 1}^{j} & w_{o 1}^{j} & \varphi_{1}^{j} & \ldots & v_{o m}^{j} & w_{o m}^{j} & \varphi_{m}^{j} & \ldots
\end{array}\right\}
\end{gathered}
$$

Vale lembrar que o índice $j$ representa o nó ativo e deve variar de 1 até o número de nuvens que cobre o domínio global do problema.

Como a dedução da matriz de rigidez e do vetor de cargas se dá na forma matricial, as modificações das funções de aproximação alteram as ordens das matrizes mas não o processo de construção. Sendo assim, obtém-se, equações idênticas às apresentadas no final do item 3.5.2:

$$
\begin{aligned}
& K=\int_{\Omega} B_{t}^{T} \cdot C \cdot B_{t} \cdot d V \\
& F=\int_{\partial \Omega_{D}} N^{T} \cdot \vec{p} \cdot d \partial \Omega
\end{aligned}
$$




\section{Programa Desenvolvido}

Como parte integrante deste trabalho foi desenvolvido um código computacional, em linguagem FORTRAN, para o cálculo de esforços e deslocamentos em estruturas de cascas axissimétricas, com geometria qualquer.

A ferramenta desenvolvida contempla a resolução dos problemas tanto do ponto de vista do MEF convencional quanto com a possibilidade da aplicação de funções de enriquecimento, baseadas no Método dos Elementos Finitos Generalizados (MEFG).

\subsection{Recursos numéricos utilizados}

Nessa seção faz-se um breve comentário sobre os recursos numéricos implementados no programa, como os tipos de partição de unidade utilizados nas aproximações, a regra para integração numérica e a rotina para resolução do sistema de equações.

\subsubsection{Partição da unidade}

Na seqüência apresentam-se as expressões e os gráficos das funções possíveis de serem utilizadas como PU dentro do programa, e suas respectivas derivadas primeira. As partições da unidade podem ser polinomiais de três graus distintos.

- Função polinomial do primeiro grau sem derivada contínua (figura 29). Partição da unidade e derivada primeira:

$$
\begin{aligned}
& P u_{i 1}(\eta)=\frac{1}{2}-\frac{1}{2} \cdot \eta \quad e \quad P u_{j 1}(\eta)=\frac{1}{2}+\frac{1}{2} \cdot \eta \\
& \frac{d P u_{i 1}(\eta)}{d \eta}=-\frac{1}{2} \quad e \quad \frac{d P u_{j 1}(\eta)}{d \eta}=\frac{1}{2}
\end{aligned}
$$

- Função polinomial do terceiro grau com derivadas contínuas (figura 30). Partição 


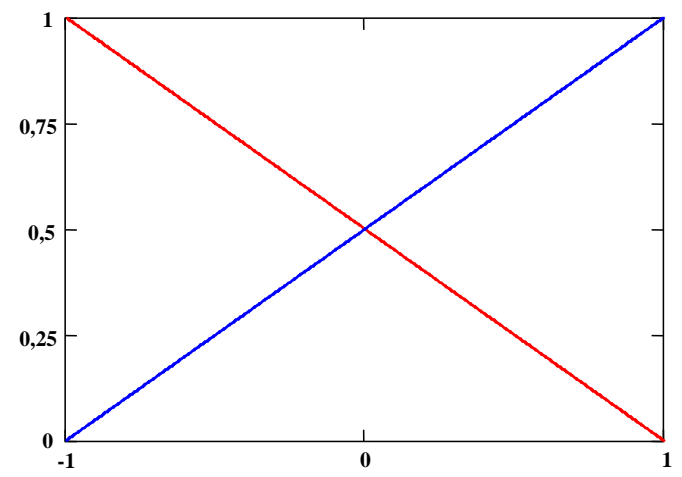

(a) Partição da unidade

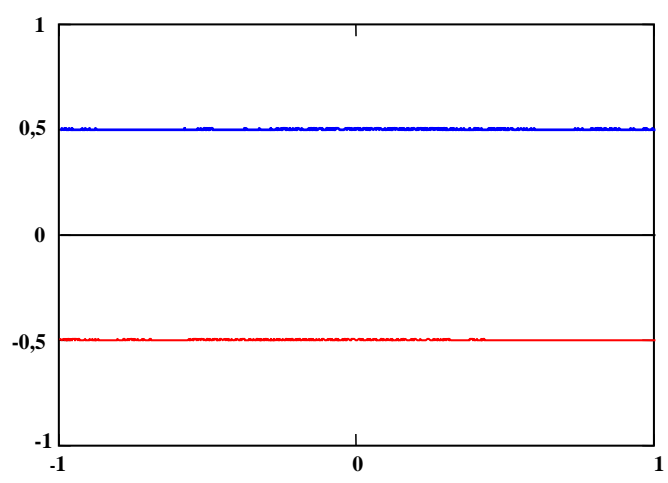

(b) Derivada primeira

Figura 29: Partição da unidade e derivada primeira - polinômio do primeiro grau da unidade e derivada primeira:

$$
\begin{array}{ccc}
P u_{i 1}(\eta)=\frac{1}{2}-\frac{3}{4} \cdot \eta+\frac{1}{4} \cdot \eta^{3} & \text { e } & P u_{j 1}(\eta)=\frac{1}{2}+\frac{3}{4} \cdot \eta-\frac{1}{4} \cdot \eta^{3} \\
\frac{d P u_{i 1}(\eta)}{d \eta}=\frac{3}{4}+\frac{3}{4} \cdot \eta^{2} & \text { e } & \frac{d P u_{j 1}(\eta)}{d \eta}=\frac{3}{4}-\frac{3}{4} \cdot \eta^{2}
\end{array}
$$

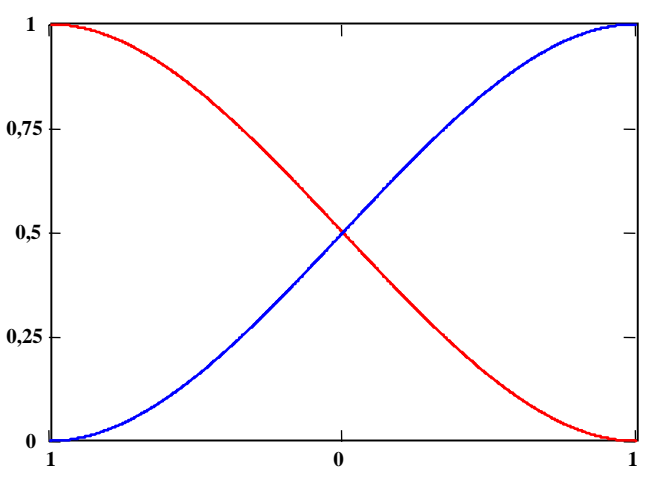

(a) Partição da unidade

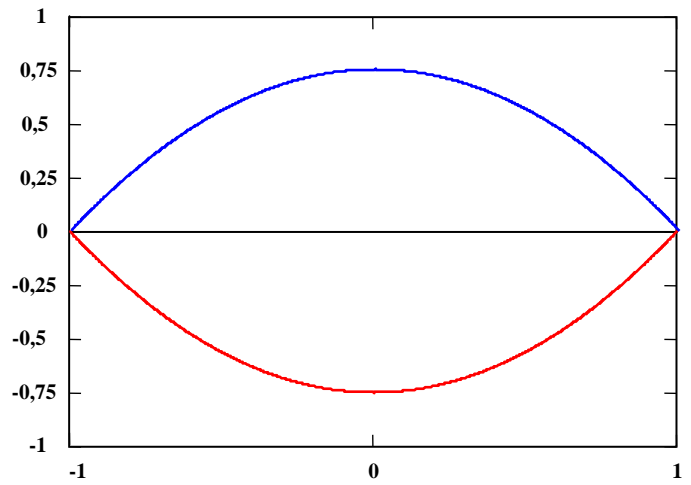

(b) Derivada primeira

Figura 30: Partição da unidade e derivada primeira - polinômio do terceiro grau

- Função polinomial do quinto grau com derivadas continuas (figura 31). Partição da unidade e derivada primeira:

$$
\begin{aligned}
& P u_{i 1}(\eta)=\frac{1}{2}-\frac{15}{16} \cdot \eta+\frac{5}{8} \cdot \eta^{3}-\frac{3}{16} \cdot \eta^{5} \quad e \quad P u_{j 1}(\eta)=\frac{1}{2}-\frac{15}{16} \cdot \eta-\frac{5}{8} \cdot \eta^{3}+\frac{3}{16} \cdot \eta^{5} \\
& \frac{d P u_{i 1}(\eta)}{d \eta}=\frac{15}{16}+\frac{15}{8} \cdot \eta^{2}-\frac{15}{16} \cdot \eta^{4} \quad e \quad \frac{d P u_{j 1}(\eta)}{d \eta}=\frac{15}{16}-\frac{15}{8} \cdot \eta^{2}+\frac{15}{16} \cdot \eta^{4}
\end{aligned}
$$




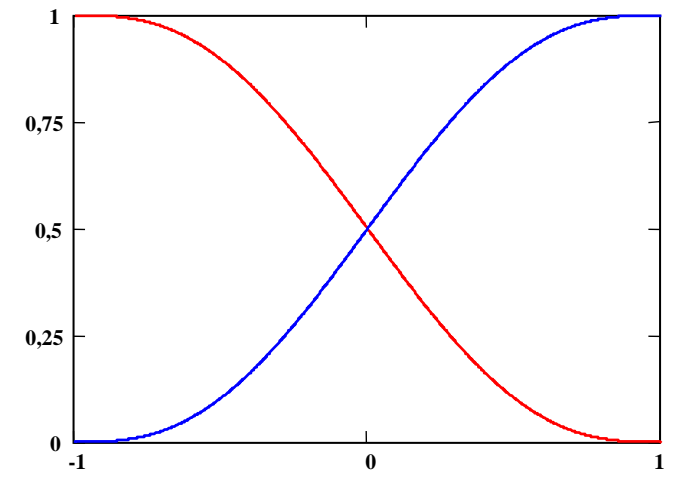

(a) Partição da unidade

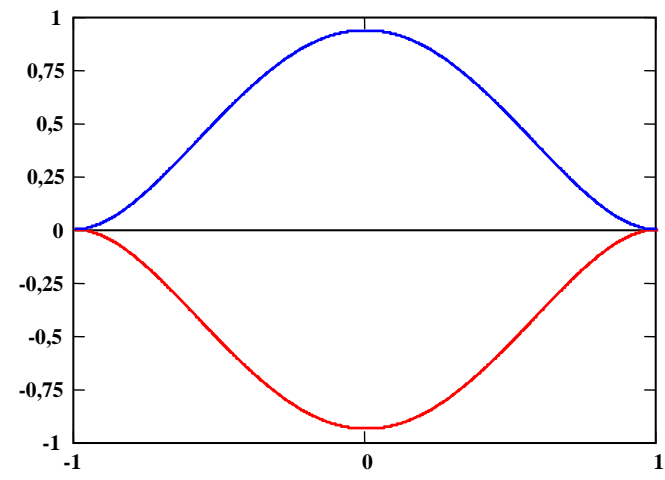

(b) Derivada primeira

Figura 31: Partição da unidade e derivada primeira - polinômio do quinto grau

As funções de forma estão vinculadas aos nós de extremidade do elemento, sendo os sub-índices $i$ e $j$ os nós inicial e final do elemento, respectivamente.

\subsubsection{Integração numérica}

Toda integração necessária é feita segundo a regra da Quadratura de GaussLegendre, onde a aproximação é representada pela seguinte expressão:

$$
\int_{a}^{b} f(x) \cdot d x \cong W_{1} \cdot f\left(x_{1}\right)+W_{2} \cdot f\left(x_{2}\right)+\ldots+W_{n-1} \cdot f\left(x_{n-1}\right)+W_{n} \cdot f\left(x_{n}\right)
$$

$W_{i}$ são os pesos associados a cada ponto de integração e $n$ é o número de pontos de integração utilizados dentro dos limites de integração.

Sabe-se que a quantidade de pontos de integração $(n)$ define o grau do polinômio que aproxima a curva a ser integrada. Tal fato possibilita que a integração seja exata, para qualquer polinômio de grau até $2 n-1$. Para qualquer curva que não seja um polinômio, a integral resulta em valor aproximado.

Por generalidade, o programa computacional prevê a possibilidade do emprego de funções não-polinomiais para o enriquecimento. Quando for esse o caso, a integração numérica é realizada dividindo-se o intervalo de integração em 4 sub-regiões, com 10 pontos de integração em cada um deles.

\subsubsection{Rotina para resolução do sistema $-K \cdot \vec{U}=\vec{F}$}

O método de enriquecimento, utilizado neste trabalho com o uso de funções polinomiais para tal, gera uma matriz de rigidez positivo semi-definida, impossibilitando, 
assim, a resolução do sistema de equações em métodos convencionais.

Para contornar e solucionar esse problema foi implementada a rotina apresentada em (DUARTE; BABUSKA; ODEN, 2000) que consiste num processo iterativo. Introduz-se uma pequena perturbação (da ordem de $1.10^{-10}$ ) na diagonal principal da matriz de rigidez global, levantando a singularidade do sistema, possibilitando que ele seja resolvido mediante correções a cada iteração, até que seja alcançada a tolerância desejada.

Na seqüência apresenta-se o algoritmo para resolução do sistema de equações, reproduzido de (BARROS, 2002).

- Algoritmo para o procedimento de Babuska:

$T_{i j}=\frac{1}{\sqrt{K_{i j}}}$

$F_{\text {nor }}=T \cdot F$

$K_{\text {nor }}=T \cdot K \cdot T \quad$ (normalização da diagonal principal)

$K_{\delta}=K_{n o r}+\delta \cdot I \quad$ (perturbação da diagonal principal)

$U_{0, \text { nor }}=K_{\text {nor }}^{-1} \cdot F_{\text {nor }} \quad$ (deslocamentos iniciais)

$\rho_{0}=F_{\text {nor }}-K_{\text {nor }} \cdot U_{0, \text { nor }} \quad$ (resíduo inicial)

$e_{0}=K_{\delta}^{-1} \cdot \rho_{0} \quad$ (erro inicial)

$i=1$

enquanto $\quad\left\|\frac{e_{i} \cdot K_{n o r} \cdot e_{i}}{U_{i, n o r} \cdot K_{n o r} \cdot U_{i, n o r}}\right\|<t o l \quad$ faça

$\rho_{i}=\rho_{0}-\sum_{j=1}^{i-1} K_{n o r} \cdot e_{j}$

$e_{i}=K_{n o r}^{-1} \cdot \rho_{i}$

$U_{i, \text { nor }}=U_{0, \text { nor }}-\sum_{j=1}^{i-1} e_{j}$

$i=i+1$

fim enquanto

$U=T \cdot U_{i, n o r}$

\subsection{Recursos do programa}

O código desenvolvido é composto por um conjunto de 94 sub-rotinas dependentes entre si.

Desde a entrada de dados até a visualização dos resultados finais, cada etapa do programa é efetuada por uma rotina diferente, possibilitando assim um melhor entendimento da seqüência de cálculos para um usuário que esteja utilizando o código. 
A seguir comentam-se os principais recursos e quais resultados podem ser obtidos com o programa.

\subsubsection{Etapas de pré-processamento e pós-processamento}

A entrada de dados é feita via leitura de arquivo de texto, tipo txt. O arquivo de entrada de dados segue determinado padrão e deve estar na mesma pasta do programa principal. A visualização dos resultados é feita através de um arquivo de texto, também com extensão txt, criado pelo código e gerado na mesma pasta onde se encontra o arquivo executável do programa.

\subsubsection{Entrada de dados}

Nessa etapa são definidos os dados do problema a ser estudado como: geometria da superfície, materiais constituintes, condições de vinculação com o meio externo, carregamentos aplicados à estrutura, forma da partição de unidade, identificação das nuvens enriquecidas e a ordem polinomial das funções de enriquecimento.

A montagem do arquivo de entrada segue o roteiro comum de um programa de elementos finitos, ficando a diferença no que diz respeito à escolha das nuvens que terão sua função de aproximação enriquecida e o grau do polinômio de enriquecimento. Para o enriquecimento nodal existe a possibilidade da escolha desde um polinômio de grau zero ao décimo quinto.

O enriquecimento das nuvens fica a critério do usuário, sendo que a escolha do polinômio de grau nulo leva à resolução do problema via MEF convencional, onde é usada apenas a Partição da Unidade como forma de aproximação da solução.

Outra opção, que fica a cargo do usuário, é a escolha do tipo da PU a ser utilizada; como já citado anteriormente no item 5.1.1, ela pode ser uma função linear, cúbica ou do quinto grau.

Os tipos de carregamento aplicados à estrutura são também escolhidos nessa etapa. O programa oferece três tipos de carregamento distribuídos: uniformemente, com variação linear e de gravidade. Há também a possibilidade de aplicação de forças e momentos concentrados, em nós ativos da estrutura. 


\subsubsection{Arquivo de resultados}

O arquivo com os resultados da análise é montado automaticamente pelo programa, após a etapa de processamento do modelo. A geração desse arquivo é feita por uma rotina, que busca os valores calculados e transfere para o arquivo de resultados da analise.

Juntamente com a apresentação dos valores de esforços normais e cortantes, momentos fletores e deslocamentos associados aos nós da estrutura, o arquivo gerado traz consigo um resumo do modelo analisado.

Para uma posterior visualização gráfica dos resultados são calculados para 10 pontos dentro do elemento, por interpolação com as funções de aproximação escolhidas, valores para os esforços e deslocamentos.

\subsubsection{Etapa de processamento}

A etapa de processamento se dá de forma interna, sem qualquer tipo de visualização ao usuário; caso haja algum erro que venha a impossibilitar a continuação da execução do programa, o mesmo mostra na tela do computador um código definido internamente para erro.

Após a conclusão da etapa de processamento os resultados obtidos são armazenados em vetores e matrizes, sendo posteriormente apresentados na forma de arquivo de texto, como já citado no item 5.2.1.

\subsubsection{Cálculo dos deslocamentos e esforços internos}

O resultado do campo de deslocamentos, vem da resolução do sistema de equações obtido no item 3.5.2.

$$
K \cdot U=F
$$

O cálculo de todos os esforços internos: momentos fletores, forças cortantes e normais é feito através da integral das tensões na espessura do elemento, utilizando-se também da integração numérica para tal. A obtenção das tensões vem dos procedimentos descritos nas seções 3.3 e 3.5, na seção 3.3 é mostrada a relação entre tensões e deformações e na seção 3.5 é descrita a relação entre deformações e deslocamentos. 


\subsubsection{Tipos de elementos implementados}

Como já comentado anteriormente, neste trabalho foram implementados dois tipos de elementos finitos para construção dos modelos, sendo que a diferença entre eles está ligada à geometria da estrutura a ser modelada.

O primeiro elemento é de aplicação mais geral, pois tem a capacidade de se adaptar a qualquer formato de curva. A aproximação da geometria é feita por segmentos de parábolas do segundo grau, que obedecem às equações citadas no capítulo 3.

O segundo elemento é usado apenas para o caso de superfícies esféricas. Nesse elemento não são usadas as funções lagrangianas mas funções trigonométricas envolvendo diretamente as coordenadas dos pontos, o raio de curvatura do elemento, o que possibilita um mapeamento exato dos pontos da casca esférica.

A escolha entre o uso de um elemento ou outro é feita na entrada de dados do modelo. Na especificação dos elementos, um dos parâmetros de entrada é o raio seu de curvatura. Caso se queria utilizar do elemento com geometria definida, o elemento circunferêncial, basta apenas especificação do raio; caso contrario o programa utiliza o elemento dito geral, sendo que esse último também se adapta ao caso das cascas esféricas.

\subsection{Fluxograma}

Esta seção é dedicada à apresentação do fluxograma básico do funcionamento do código desenvolvido. Os passos aqui apresentados visam mostrar a seqüência básica de procedimentos executados pelo programa. 


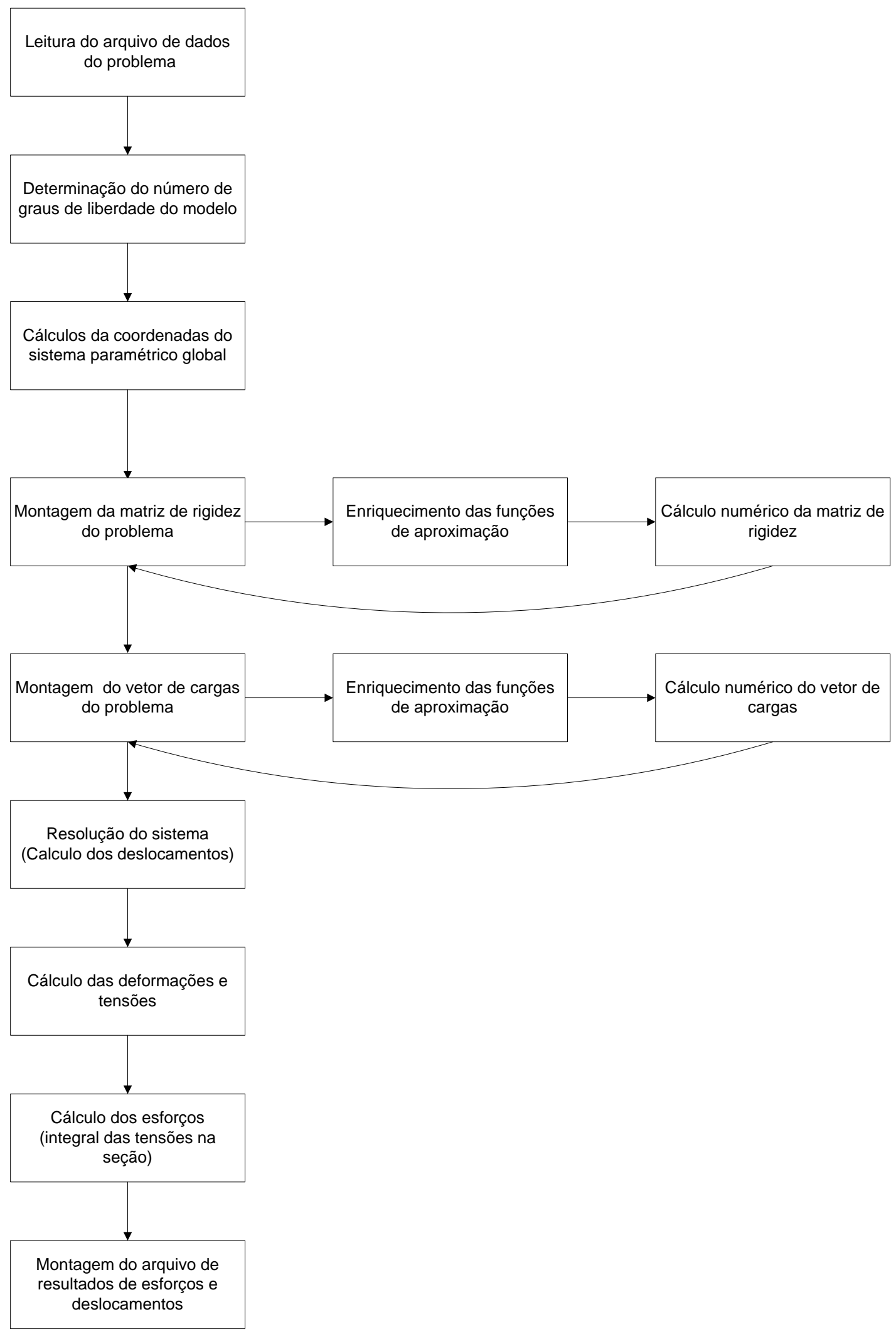

Figura 32: Fluxograma básico do programa desenvolvido 


\section{Exemplos Numéricos}

Neste capítulo demonstra-se a utilização da técnica de enriquecimento na resolução de problemas de cascas de revolução.

Os seis exemplos mostrados visam comprovar a eficiência do MFEG na determinação dos esforços e deslocamentos das estruturas, por comparação com uma solução de referência obtida pelo Método dos Elementos Finitos convencional com refino h da rede, bastante elevado.

O processo de refino h foi empregado nos seis exemplos para fins de estudo comparativo de convergência neste capítulo. As redes de elementos finitos foram formadas da seguinte maneira: para o refino h foram montadas redes com 2, 10, 20, 50, 100, 200, 500 e 1000 elementos sendo essa última adotada solução de referência para os exemplos das seções 6.3 e 6.7. O refino p foi aplicado somente sobre as redes de 10 e 20 elementos, propostas para o refino h, sendo a de 10 combinada com o refino p nos três primeiros exemplos e a de 20 nos três últimos. Para tal refino fez-se a utilização de polinômios de ordem $1,3,5,7,10,12$ e 15 como função de enriquecimento.

Para os códigos adotados nas legendas dos gráficos apresentados no decorrer desta sessão adotaram-se:

M10 - Rede com dez elementos finitos

M20 - Rede com vinte elementos finitos

M7 - Função de enriquecimento: polinômio do sétimo grau

Como o foco principal do trabalho é a utilização do refino p, os resultados obtidos exclusivamente com as redes montadas mediante o refino h não são mostrados na seqüência do trabalho. Tais valores são utilizados apenas para montagem das curvas de convergência nos gráficos de erro, que constam no final de cada seção, mostrando a evolução dos resultados em função do tipo de refino adotado para análise. 


\subsection{Norma energia do erro}

Para análise de convergência dos resultados e montagem dos respectivos diagramas, utilizou-se da norma que representa a média dos erros locais ao quadrado (SZABÓ; BABUSKA, 1991; PROENÇA, 2006), definida por:

$$
\|e\|=\sqrt{\int_{\Omega} e_{i}^{2} d x}
$$

Devido ao procedimento utilizado para o cálculo ser puramente numérico, a expressão que define a norma passa a ser a seguinte:

$$
\|e\|=\sqrt{\sum_{i=1}^{n} e_{i}^{2} \Delta x}
$$

sendo $n$ o número de nós da rede, $\Delta x$ a área de influência do nó e $e_{i}$ o erro local definido da seguinte forma:

$$
e_{i}=E_{i}^{r e f}-\tilde{E}_{i}
$$

onde $E_{i}^{r e f}$ e $\tilde{E}_{i}$ são os valores da solução de referência e aproximada, respectivamente.

\subsection{Exemplo 01 - Placa circular}

O exemplo mostrado nesta seção visa, em primeiro lugar, destacar a capacidade de aproximação do elemento utilizado.

Além da boa representação dos esforços e deslocamentos, procura-se também mostrar que o MEFG oferece a possibilidade de contornar o problema do travamento.

Problemas de travamento ocorrem em particular nos modelos de primeira ordem de Reissner-Mindlin, mas podem decorrer da forte distorção da rede de elementos finitos ou de um mau condicionamento da matriz de rigidez (SUAREZ; PROENÇA, 2006).

Nos modelos de Reissner-Mindlin a questão está ligada ao mecanismo de flexão. O fenômeno do travamento é o aparente ganho de rigidez da estrutura na medida que a sua espessura tende para zero. Tal fato é explicado observando-se a variação da energia da deformação, em particular a parcela que inclui a contribuição do cisalhamento. Nessas condições, reduzindo-se a espessura do elemento estrutural, a energia de deformação de- 
vida à flexão tende para zero mais rapidamente do que a cisalhante. Por conseqüência da incapacidade das aproximações usuais para reproduzir esse fato, a solução converge para uma resposta mais rígida que a real, fato esse incorreto, visto que, com a diminuição da espessura, a rigidez da estrutura deveria se reduzir (NIRSCHL; GARCIA; PROENÇA, 2005).

A aproximação normalmente adotada para o campo de deslocamentos não é, portanto, suficientemente "flexível" para reproduzir corretamente a parcela de energia de distorção.

O problema escolhido para análise do travamento consiste numa placa circular engastada (nó 2001 da figura 33) e submetida a carregamento uniformemente distribuído. As demais características do problema são mostradas na tabela 1.

Tabela 1: Exemplo 01 - Dados de entrada do modelo

\begin{tabular}{c|c}
\hline \hline Espessura constante para toda estrutura & $6 \mathrm{~cm}$ \\
\hline Modulo de elasticidade do material & $2500 \mathrm{kN} / \mathrm{m}^{2}$ \\
\hline Coeficiente de Poisson & 0,2 \\
\hline Raio da placa & $6 \mathrm{~m}$ \\
\hline Carga distribuída & $2 \mathrm{kN} / \mathrm{m}^{2}$ \\
\hline
\end{tabular}

Na figura 33 é mostrado um corte esquemático da estrutura analisada e suas dimensões.

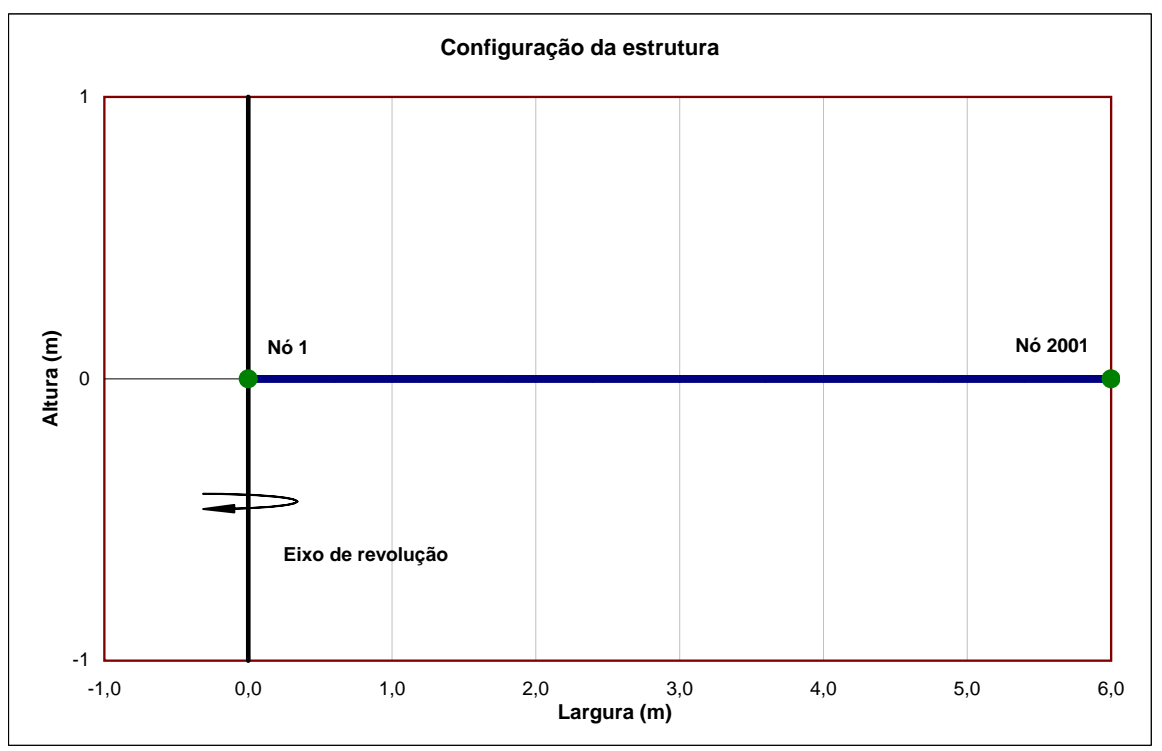

Figura 33: Exemplo 01 - Corte esquemático da estrutura

Os resultados apresentados na seqüência referem-se aos momentos fletores, tangencial $\left(M_{\theta}\right)$, radial $\left(M_{\varphi}\right)$, forças cortantes $\left(V_{\varphi}\right)$ e deslocamentos transversais $(w)$. 
Para esse exemplo a solução de referência é a solução analítica do problema tendo seus esforços e deslocamentos calculados com as seguintes expressões:

- Momento fletor tangencial $\left(M_{\theta}\right)$

$$
M_{\theta}=\frac{p}{16} \cdot\left[R^{2} \cdot(1+\nu)-r^{2} \cdot(3+\nu)\right]
$$

- Momento fletor radial $\left(M_{\varphi}\right)$

$$
M_{\varphi}=\frac{p}{16} \cdot\left[R^{2} \cdot(1+\nu)-r^{2} \cdot(1+3 \cdot \nu)\right]
$$

- Esforço cortante $\left(V_{\varphi}\right)$

$$
V_{\varphi}=\frac{p \cdot r}{2}
$$

- Deslocamento transversal $(w)$

$$
w(r)=\frac{3 \cdot p \cdot\left(1-\nu^{2}\right)}{16 \cdot E \cdot t^{3}} \cdot\left(R^{2}-r^{2}\right)^{2}
$$

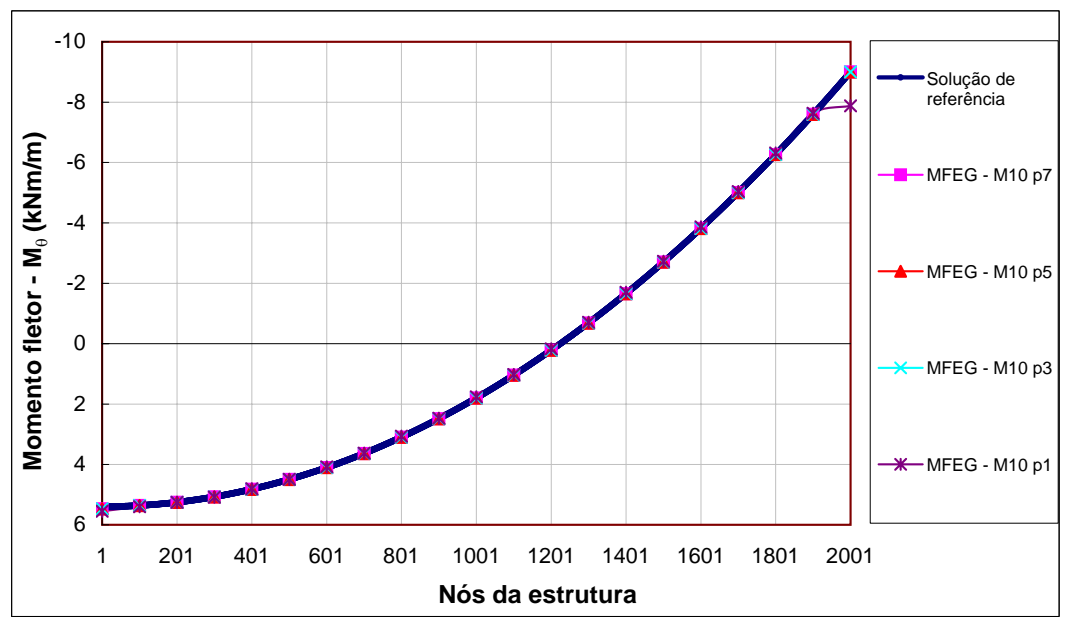

Figura 34: Momentos fletores $M_{\theta}$ - Solução de referência e refino p

Os gráficos das figuras 34 e 35 mostram que é possível obter bons resultados explorando o enriquecimento sobre uma rede reduzida de elementos. Essa conclusão é respaldada pela análise de convergência das figuras 38 e 39.

É preciso observar, em primeiro lugar, que se trata de um problema com boa regularidade dos campos de deslocamento e de momentos, sendo de menor regularidade 


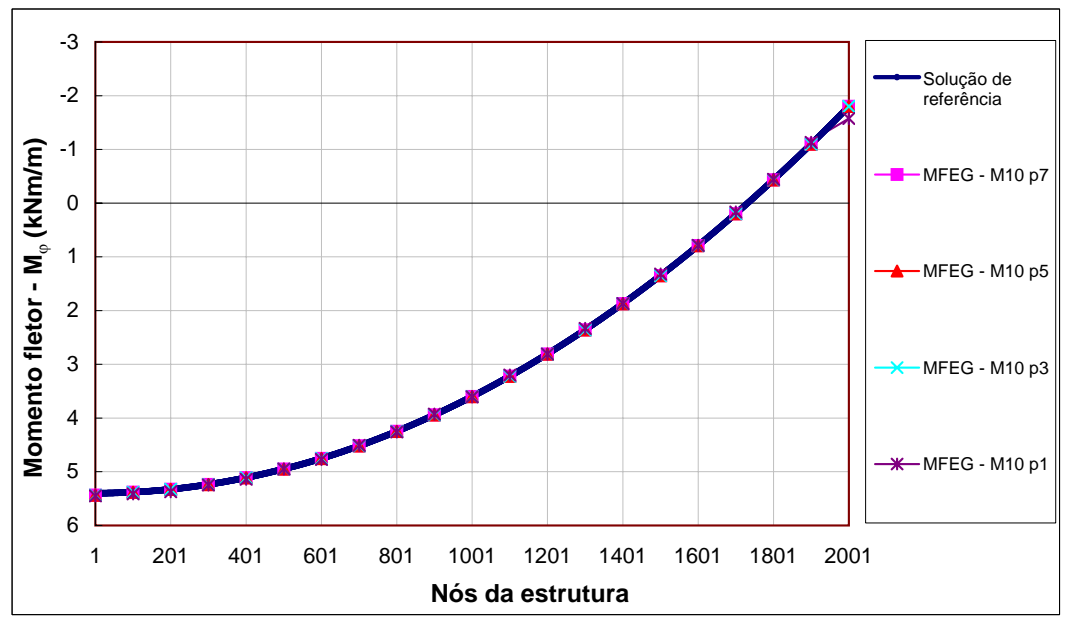

Figura 35: Momentos fletores $M_{\varphi}$ - Solução de referência e refino p

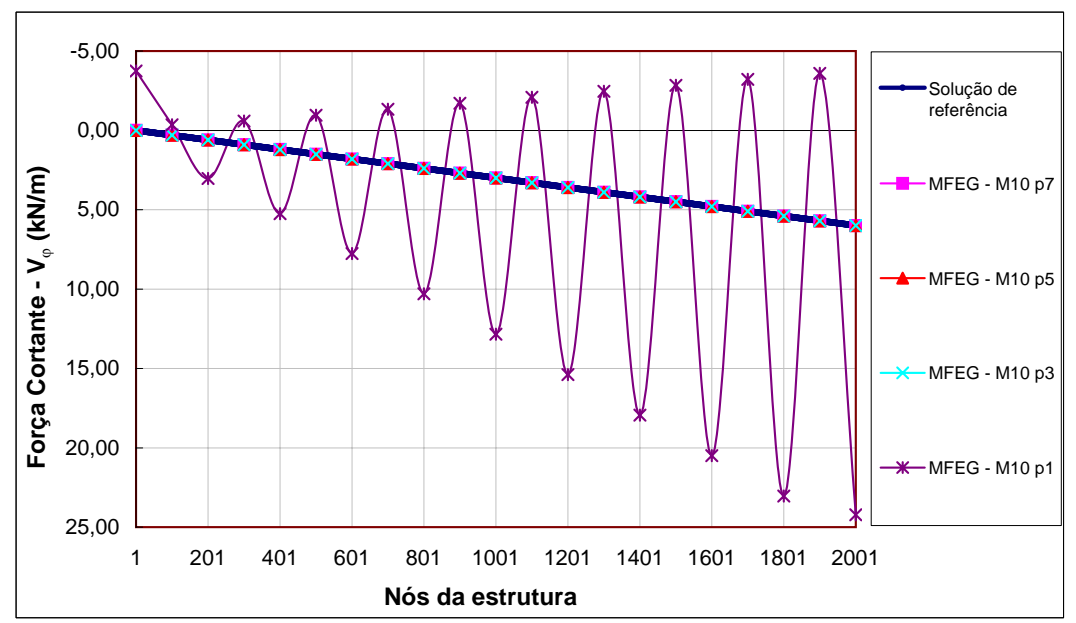

Figura 36: Forças cortantes $V_{\varphi}$ - Solução de referência e refino p

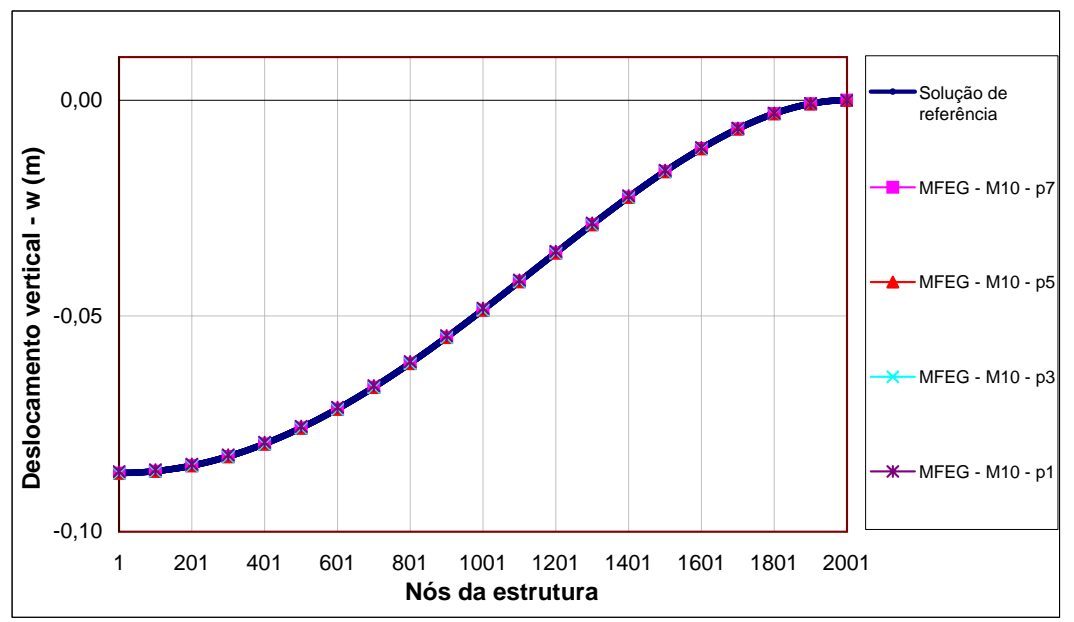

Figura 37: Deslocamentos $w$ - Solução de referência e refino p 
no esforço cortante.

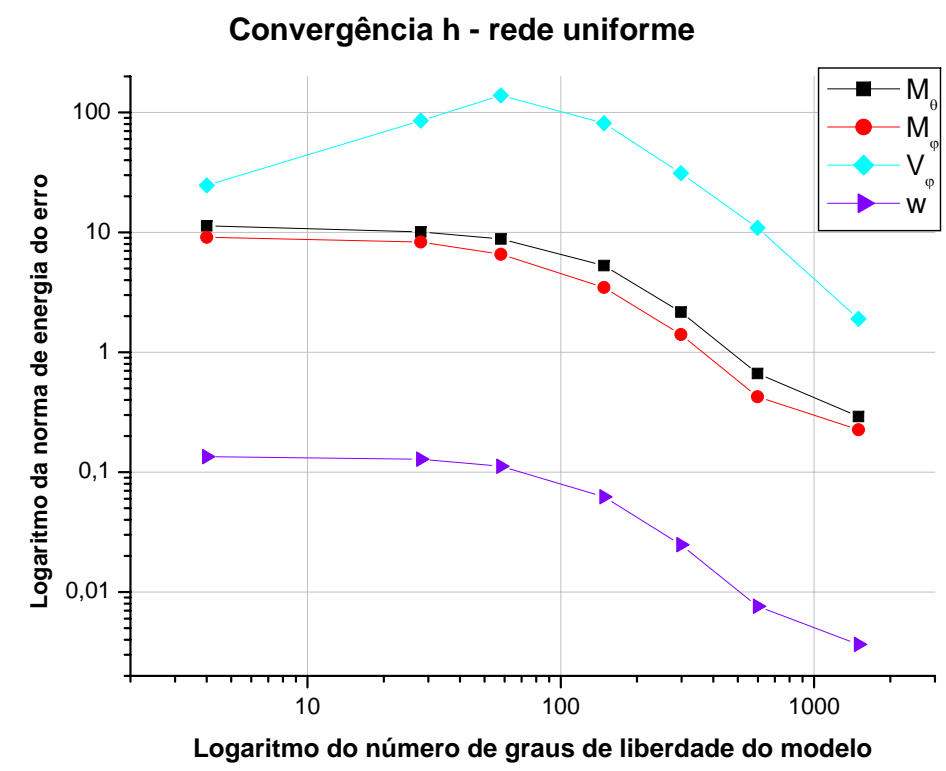

Figura 38: Convergência - refino $h$ da rede

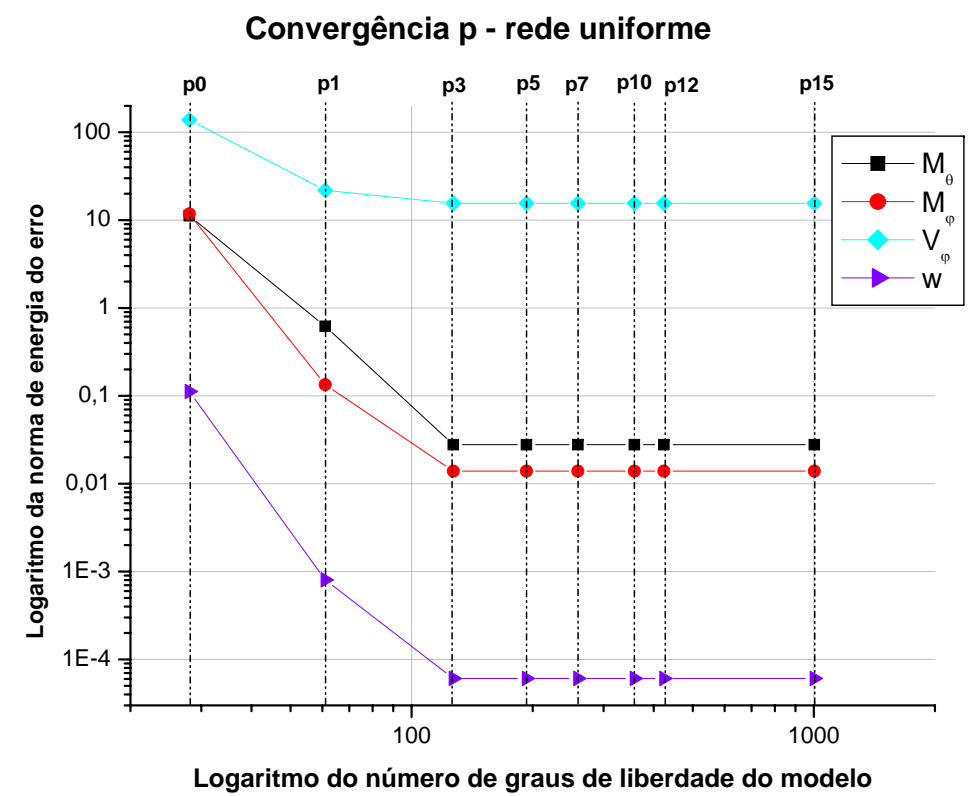

Figura 39: Convergência - refino $p$

Nota-se claramente que o refino p é bastante efetivo, por isso a taxa de convergência obtida pelo MEFG é muito superior à mesma alcançada pelo MEF convencional. Entretanto, se o objetivo for o de reduzir o erro na cortante são necessárias redes com muitos elementos. 


\subsubsection{Análise do travamento}

Para o estudo do travamento fez-se, sobre a placa do exemplo em questão, a redução da sua espessura. Com isso para cada nova situação foi feita a comparação entre a solução obtida através do MEFG e a solução analítica para placa engastada, no ponto de deslocamento máximo da estrutura, o centro da placa.

A expressão que descreve o deslocamento numa placa circular é mostrada na equação 6.7. Para o ponto de deslocamento máximo, centro da placa, tem-se $r$ nulo, logo a expressão para tal ponto fica:

$$
w_{\max }=\frac{3 \cdot p \cdot R^{4}}{16 \cdot E \cdot t^{3}} \cdot\left(1-\nu^{2}\right)
$$

No gráfico da figura 40 pode-se perceber que as soluções pelo MEFG são praticamente coincidentes entre si, e dentro de uma faixa de valores da relação $2 R / t$, o resultado é muito próximo da solução analítica. Dessa forma, o fenômeno do travamento não foi fator preocupante para as aplicações deste trabalho, visto que os exemplos apresentados têm valores para relação $2 R / t$ dentro da faixa que o MFEG consegue contornar o problema do travamento numérico.

Devido a rápida convergência dos resultados obtidos pelo MEFG, para solução analítica utilizou-se, para analisar o travamento, redes com 5, 8 e 10 elementos finitos e variou-se o grau do polinômio de enriquecimento das nuvens. Os resultados obtidos foram comparados da seguinte forma: de posse da solução analítica do problema, fez-se a normalização dos resultados em relação a mesma, ou seja, dividiu-se o valor obtido com o MEFG pelo analítico. O parâmetro escolhido para essa analise foi o deslocamento transversal no centro da placa $\left(w_{\max }\right)$.

No gráfico da figura $40 w_{a}$ é o deslocamento obtido com a solução analítica, $R$ e $t$ (variável do problema) são o raio e espessura da placa, respectivamente.

No exemplo desta seção, a relação entre o diâmetro da placa e a sua espessura $(2 R / t)$ é 200 , dentro da faixa de valores em que a solução atingida pelo MEFG converge para solução analítica do problema, fato que pode ser visto nos diagramas de esforços e deslocamentos da placa. 


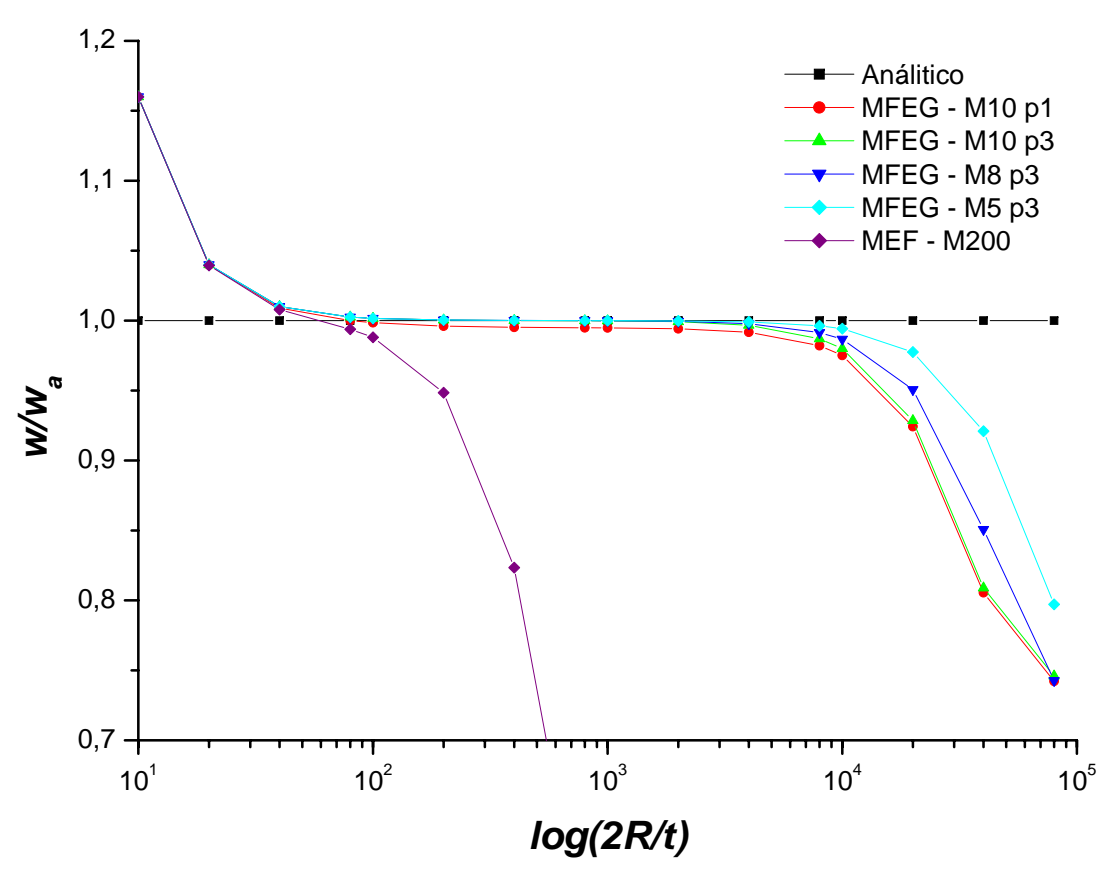

Figura 40: Travamento - Valores normalizados para o deslocamento transversal

\subsection{Exemplo 02 - Cúpula esférica abatida}

A cúpula esférica está submetida a carga distribuída tipo peso próprio e vinculada ao meio externo, mediante engaste fixo da borda (nó 2001 na figura 41). As demais características do problema são as seguintes:

Tabela 2: Exemplo 02 - Dados de entrada do modelo

\begin{tabular}{c|c}
\hline \hline Espessura constante para toda estrutura & $6 \mathrm{~cm}$ \\
\hline Módulo de elasticidade do material & $2500 \mathrm{kN} / \mathrm{cm}^{2}$ \\
\hline Coeficiente de Poisson & 0,2 \\
\hline Raio de curvatura da superfície esférica & $6 \mathrm{~m}$ \\
\hline Carga de gravidade & $2 \mathrm{kN} / \mathrm{m}^{3}$ \\
\hline
\end{tabular}

Na figura 41 é mostrado um corte esquemático da estrutura analisada e suas dimensões.

A solução de referência foi obtida com uma rede de 1000 elementos finitos. Para construção das soluções obtidas com o MEFG foi utilizado o refino p em todas as nuvens que cobrem o domínio do problema. Comparam-se com essa solução as soluções de refinos tipo h e p, de acordo com o exposto na introdução deste capítulo. 


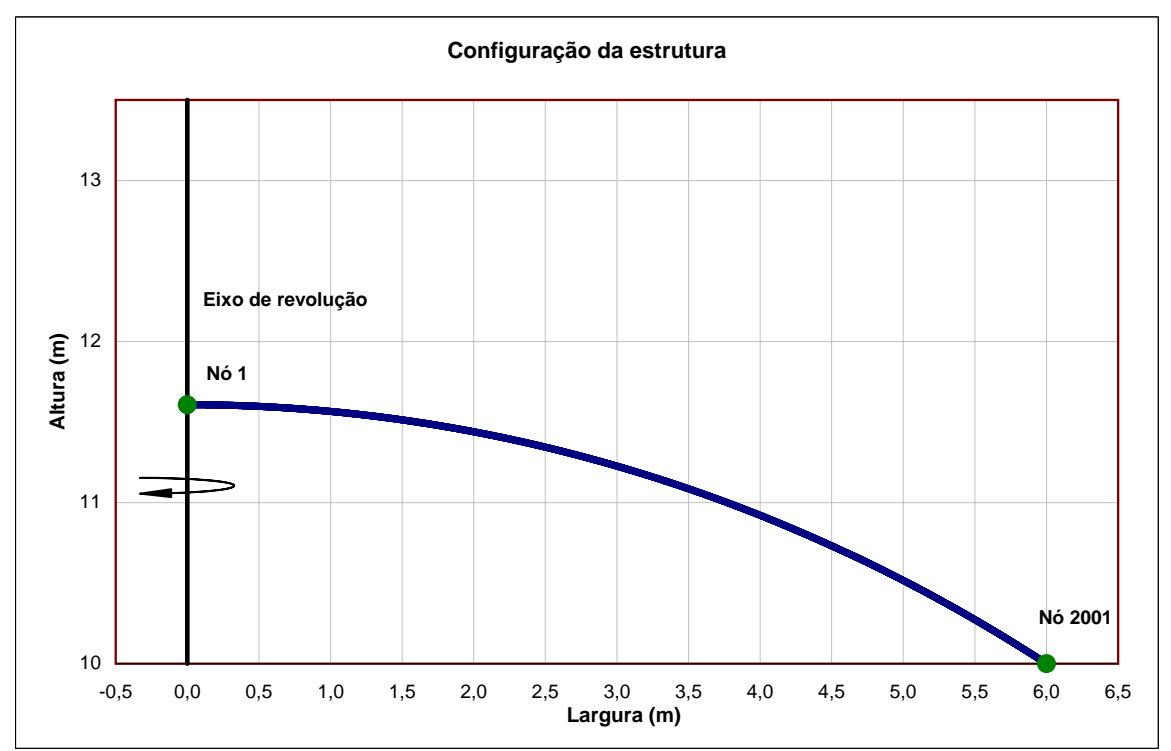

Figura 41: Exemplo 02 - Corte esquemático da estrutura

As soluções utilizando-se do refino h foram usadas para a montagem dos diagramas de convergência, apresentados ao final da seção.

Os resultados obtidos para o exemplo em questão são mostrados na seqüência de figuras 42 a 48 contendo as curvas de esforços e deslocamentos.

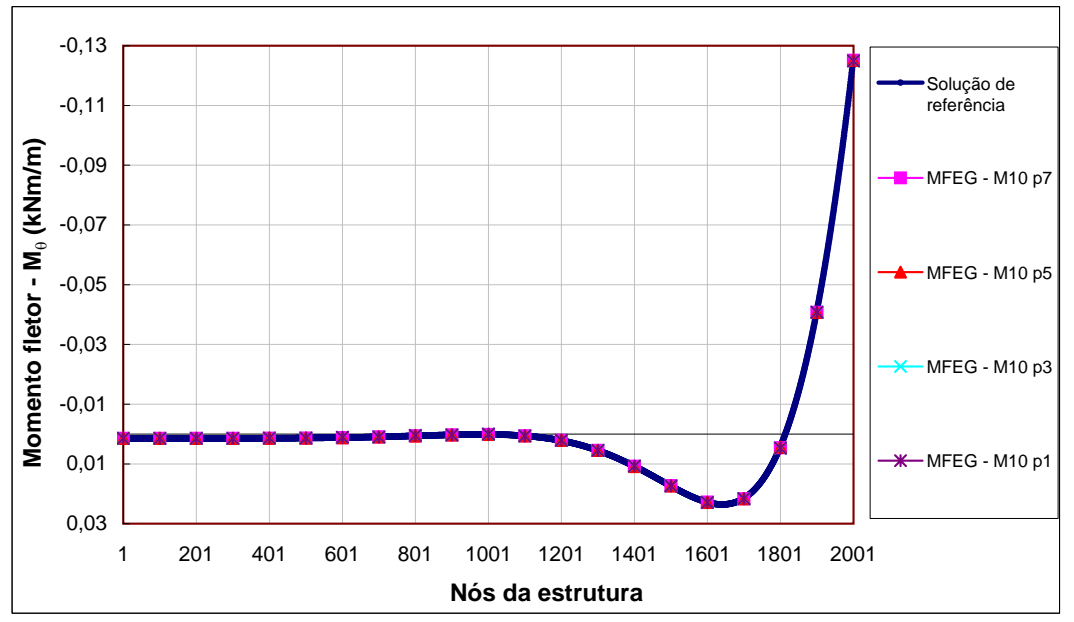

Figura 42: Momentos fletores $M_{\theta}$ - Solução de referência e refino p

Com a visualização dos resultados é possível perceber que a rede de 10 elementos e um refino p de pequena ordem fornece boa concordância com a solução de referência, exceto na força cortante $V_{\varphi}$ e força normal anular $N_{\theta}$. Para os dois últimos casos citados, o polinômio de enriquecimento do primeiro grau não é suficiente para se atingir bons resultados. Entretanto, já com enriquecimento polinomial do terceiro grau praticamente se atinge a solução de referência baseada no MEF convencional. 


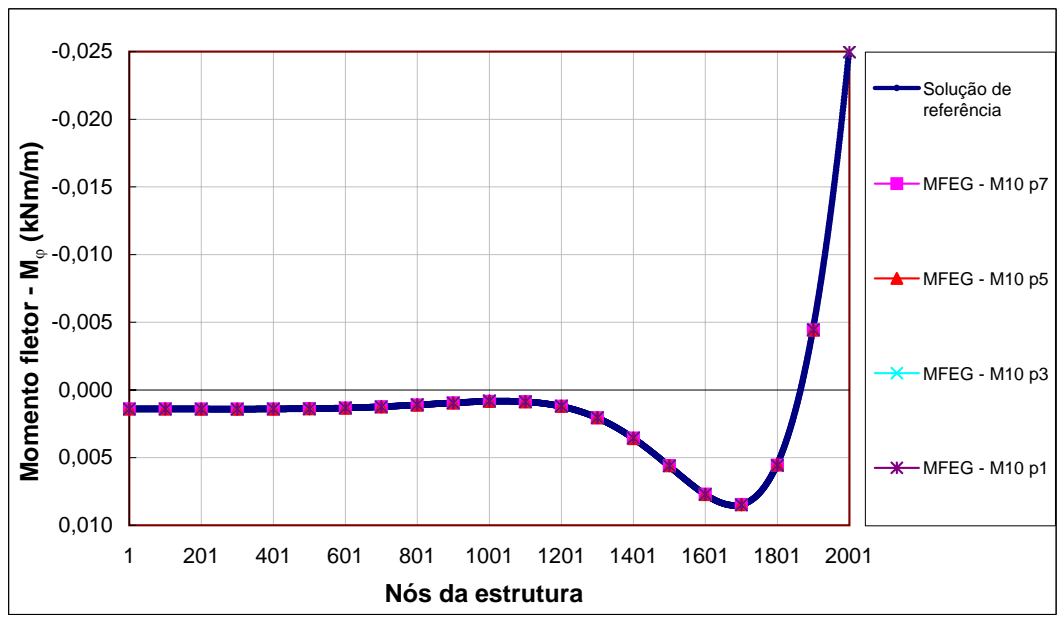

Figura 43: Momentos fletores $M_{\varphi}$ - Solução de referência e refino p

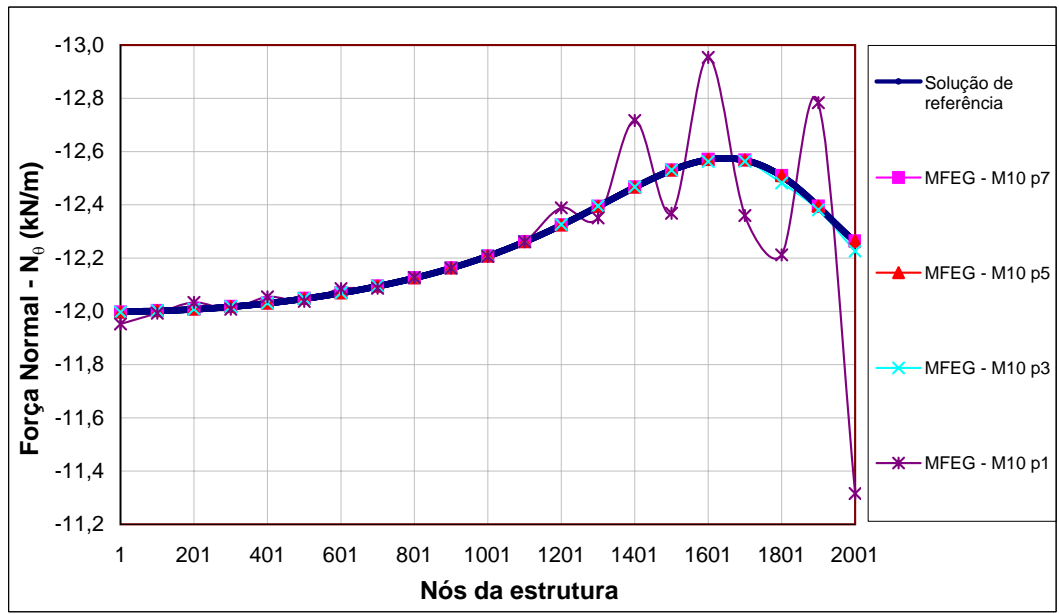

Figura 44: Forças normais $N_{\theta}$ - Solução de referência e refino p

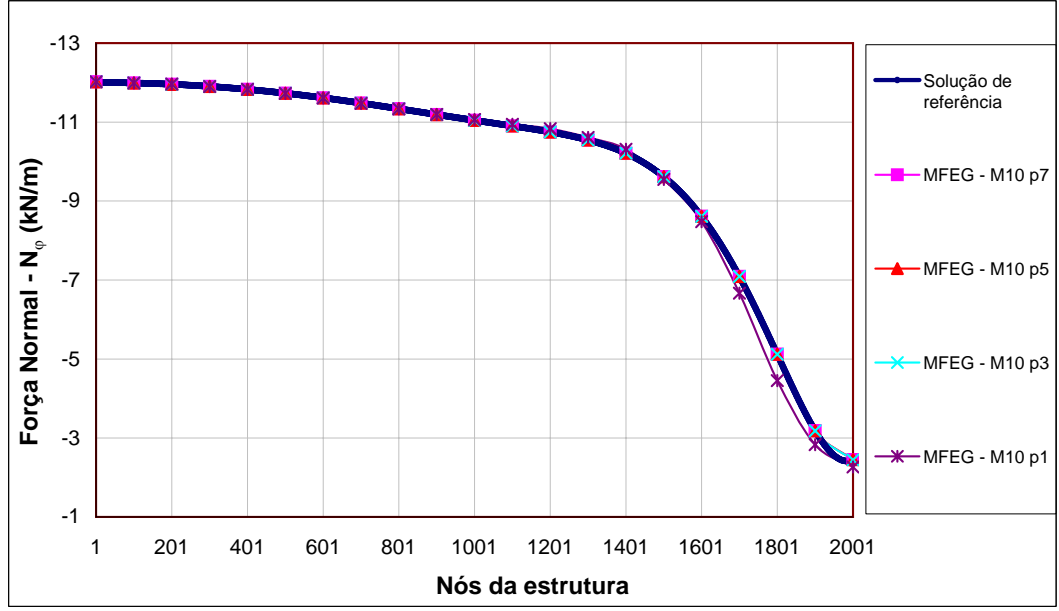

Figura 45: Forças normais $N_{\varphi}$ - Solução de referência e refino p 


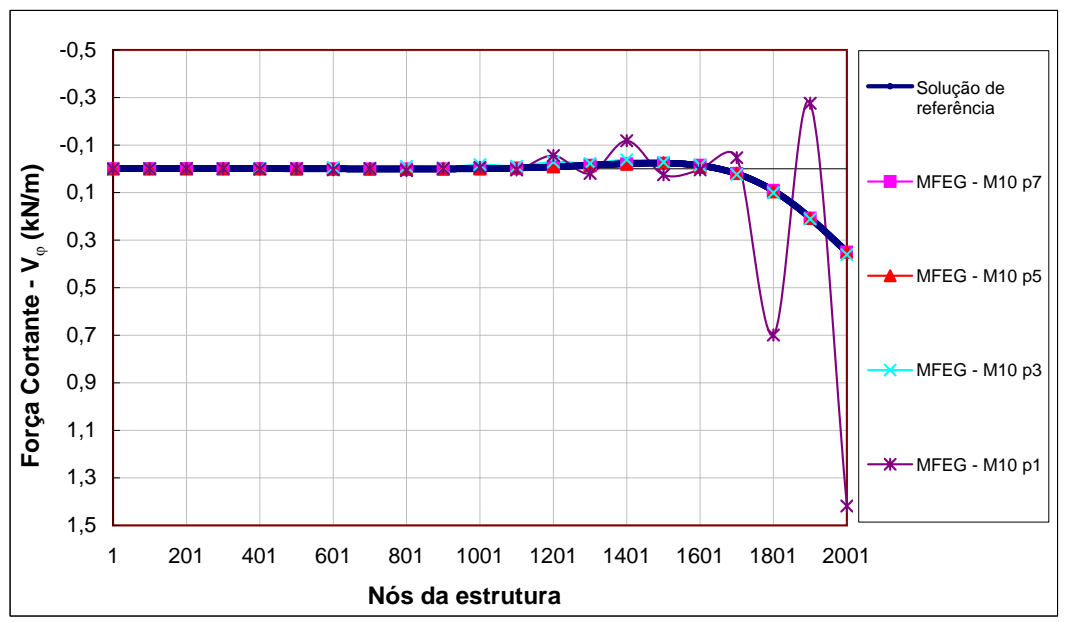

Figura 46: Forças cortantes $V_{\varphi}$ - Solução de referência e refino p

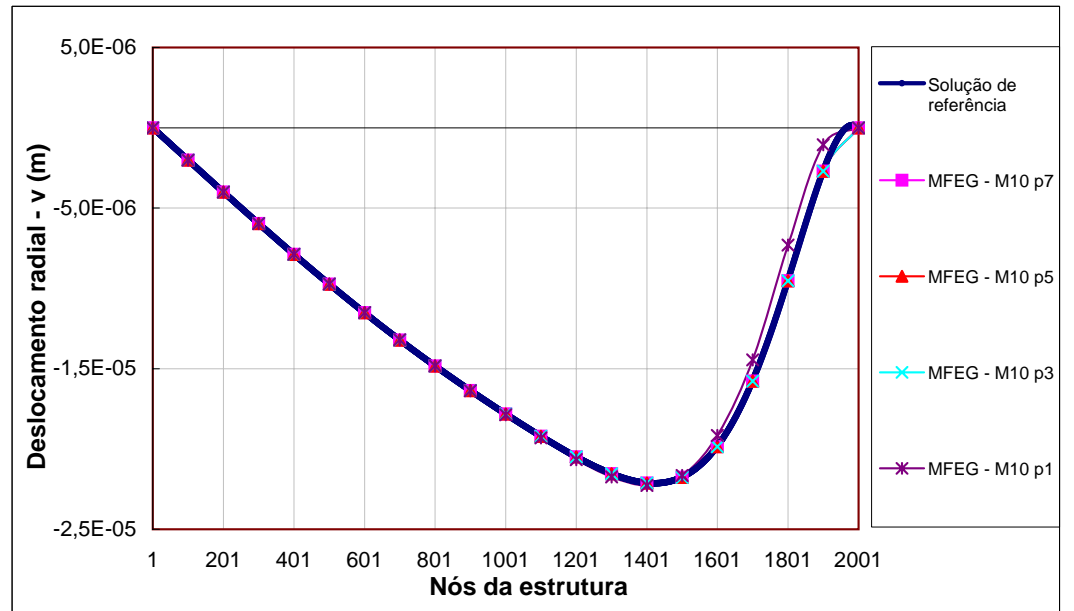

Figura 47: Deslocamentos $v$ - Solução de referência e refino p

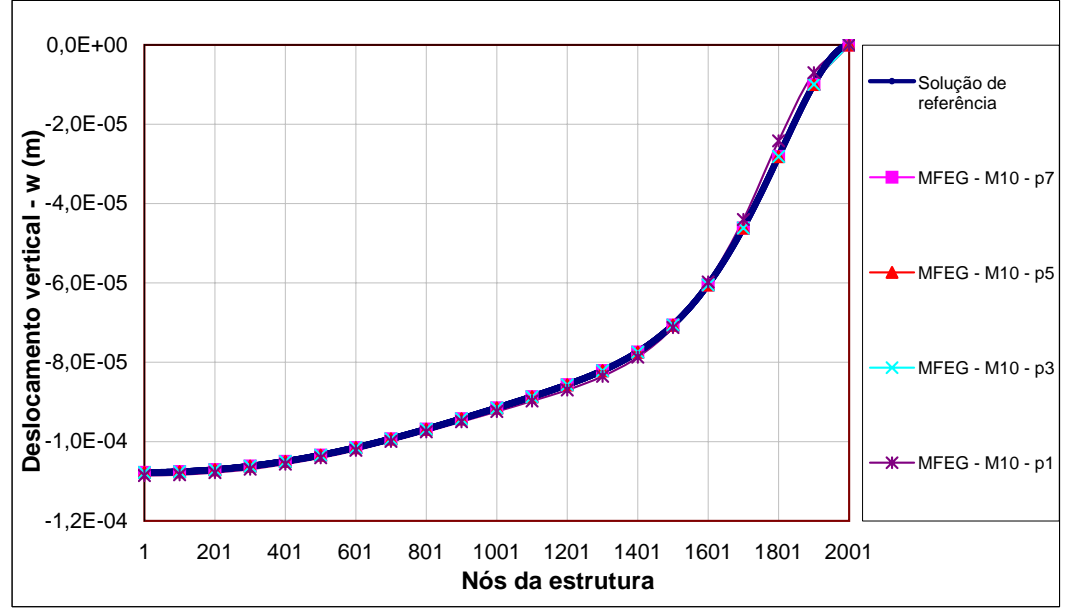

Figura 48: Deslocamentos $w$ - Solução de referência e refino p 


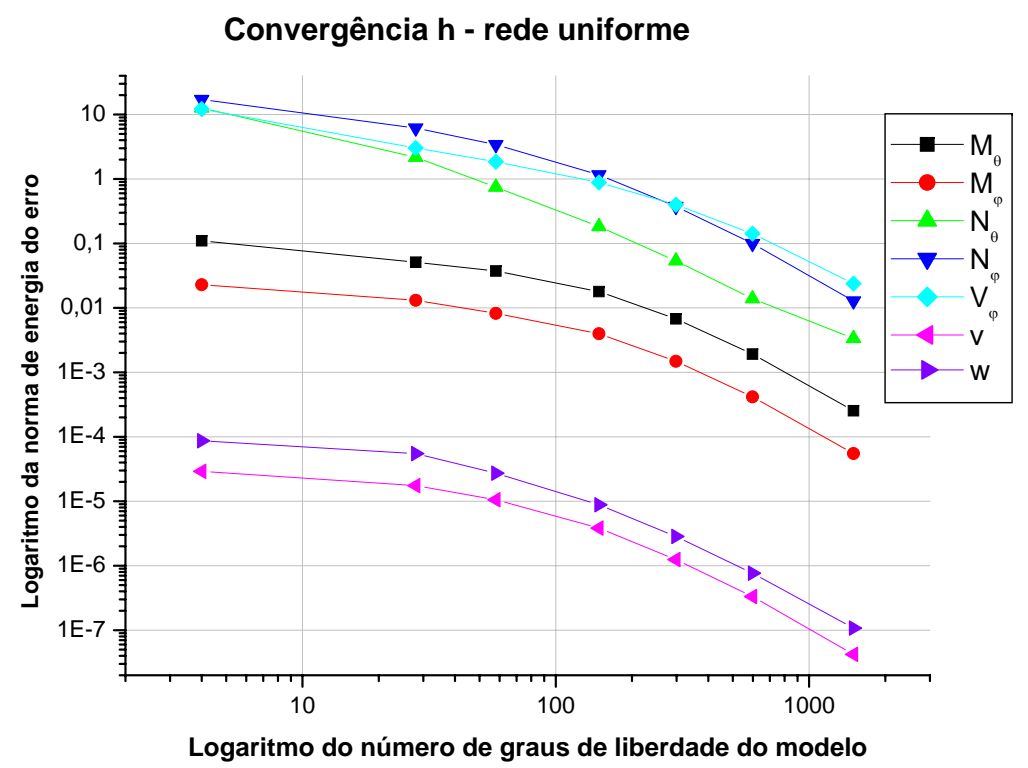

Figura 49: Convergência - refino $h$ da rede

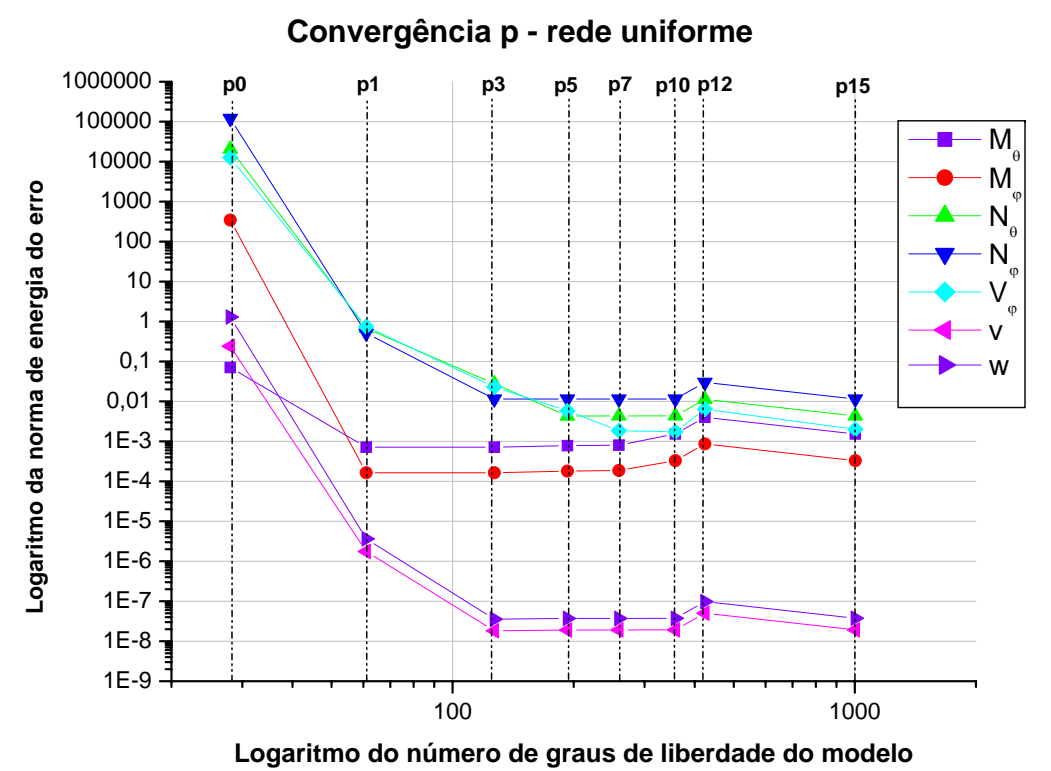

Figura 50: Convergência - refino $p$

A aparente eficiência do método utilizado pode ser confirmada pelos gráficos de convergência da solução segundo os refinos h e p, mostrados nas figuras 49 e 50 . Claramente as funções de enriquecimento aceleram bastante o processo de convergência já com uma pequena elevação na ordem polinomial das funções de aproximação. Entretanto um maior ganho de precisão somente é possivel com a combinação de processos h e p. 


\subsection{Exemplo 03 - Cilindro sob pressão}

O problema que segue trata da análise de um cilindro submetido a pressão interna. Nesse problema, mais uma vez buscou-se verificar a capacidade do método de reproduzir as variações nos esforços solicitantes atuantes nesse tipo de estrutura.

O cilindro possui espessura constante e vinculação com o meio exterior mediante engaste das extremidades da estrutura (nós 1 e 2001 na figura 51). A vinculação escolhida é para forçar o aparecimento de variação nos esforços de flexão de forma mais significativa. As demais características do problema estão citadas na tabela 3 .

Tabela 3: Exemplo 03 - Dados de entrada do modelo

\begin{tabular}{c|c}
\hline \hline Espessura constante para toda estrutura & $2 \mathrm{~mm}$ \\
\hline Modulo de elasticidade do material & $20500 \mathrm{kN} / \mathrm{cm}^{2}$ \\
\hline Coeficiente de Poisson & 0,3 \\
\hline Raio do cilindro & $10 \mathrm{~cm}$ \\
\hline Pressão interna & $0,02 \mathrm{kN} / \mathrm{cm}^{2}$ \\
\hline
\end{tabular}

Na figura 51, mostra-se um corte esquemático da superfície analisada e suas dimensões.

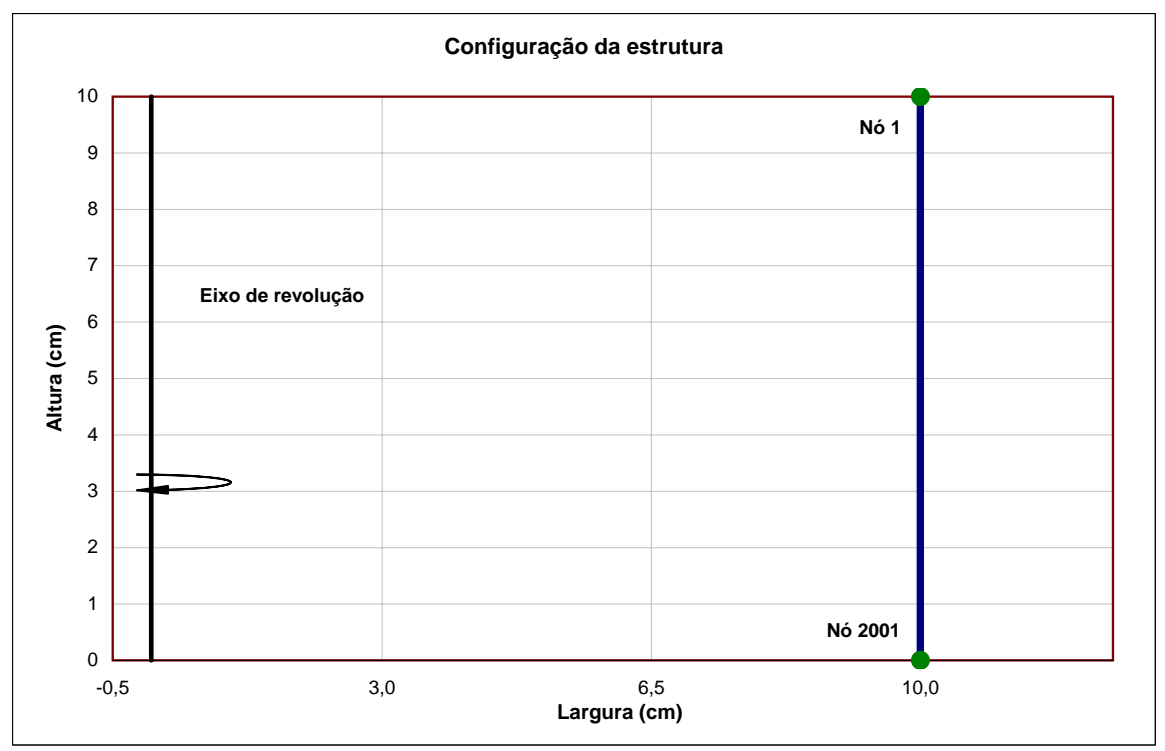

Figura 51: Exemplo 03 - Corte esquemático da estrutura

Os resultados foram obtidos com refino p aplicado a todas as nuvens sendo apresentados na seqüência de figuras 52 a 58 onde mostram-se os diagramas de esforços e deslocamentos do problema em questão. 


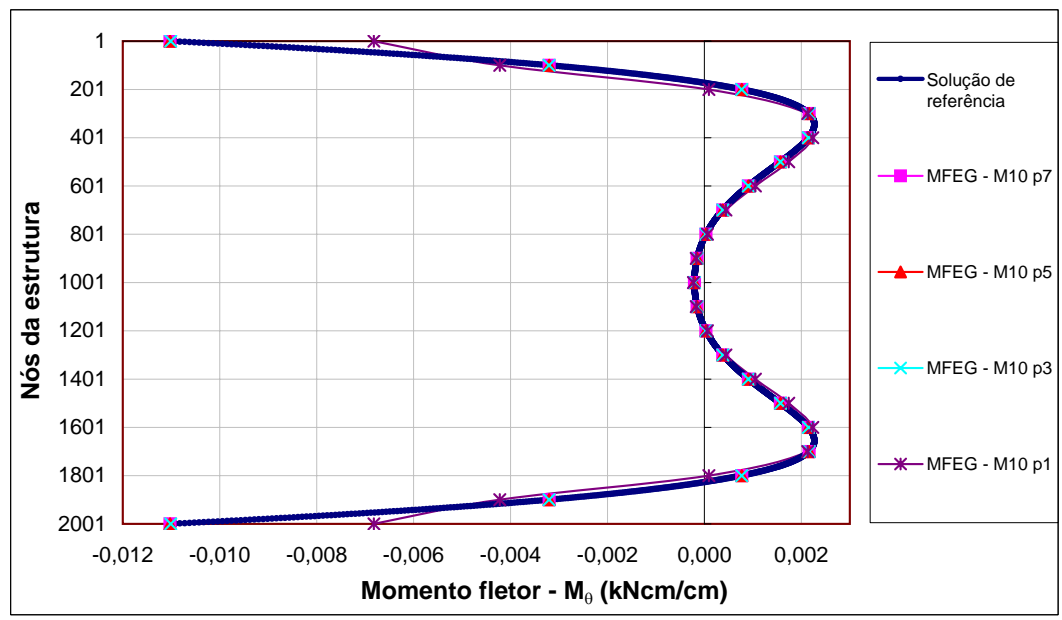

Figura 52: Momentos fletores $M_{\theta}$ - Solução de referência e refino p

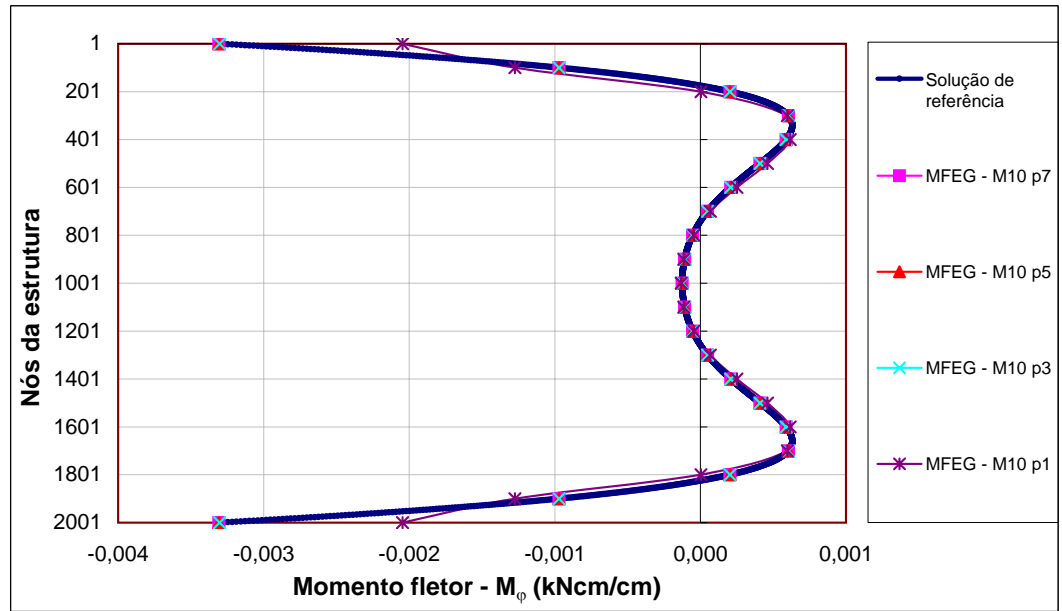

Figura 53: Momentos fletores $M_{\varphi}$ - Solução de referência e refino p

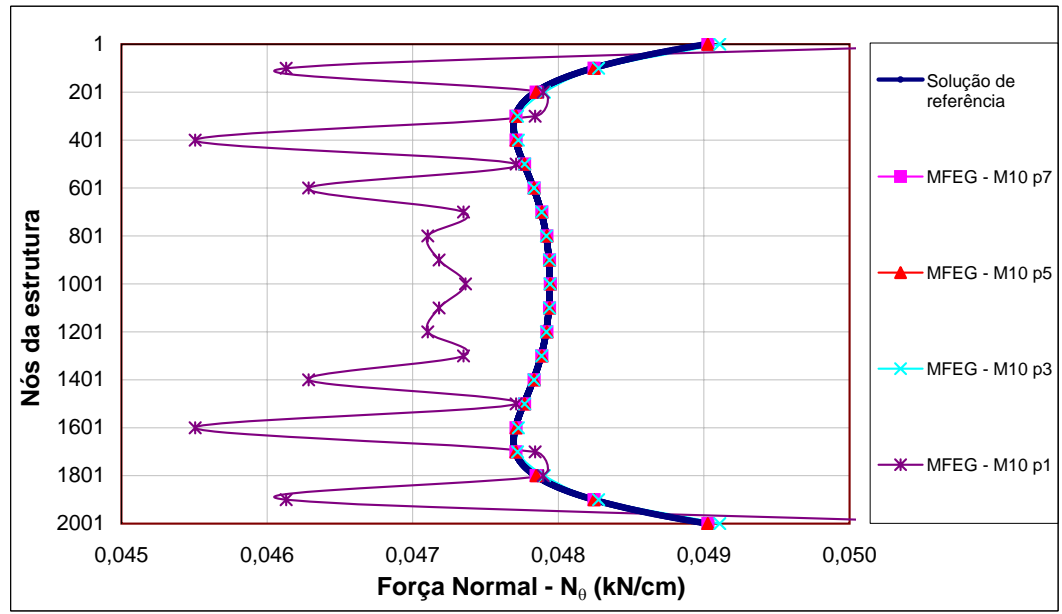

Figura 54: Forças normais $N_{\theta}$ - Solução de referência e refino p 


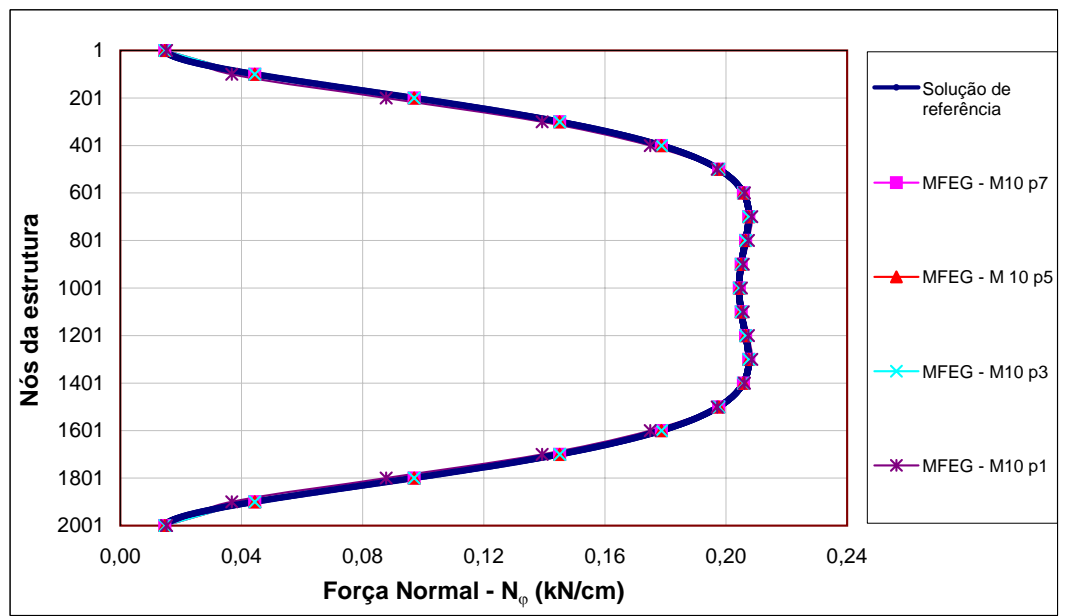

Figura 55: Forças normais $N_{\varphi}$ - Solução de referência e refino p

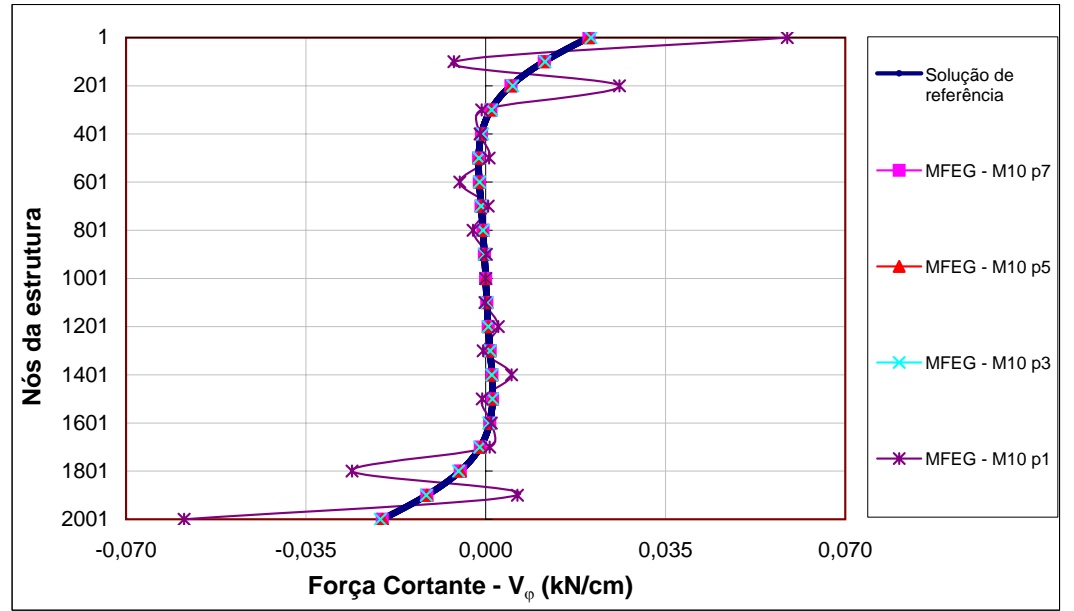

Figura 56: Forças cortantes $V_{\varphi}$ - Solução de referência e refino p

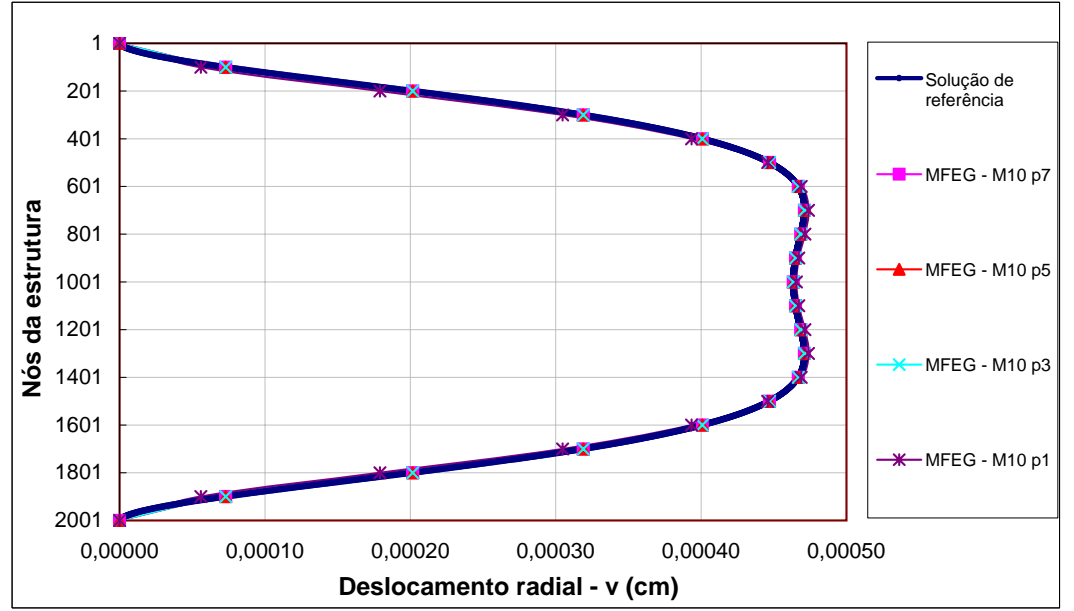

Figura 57: Deslocamentos $v$ - Solução de referência e refino p 


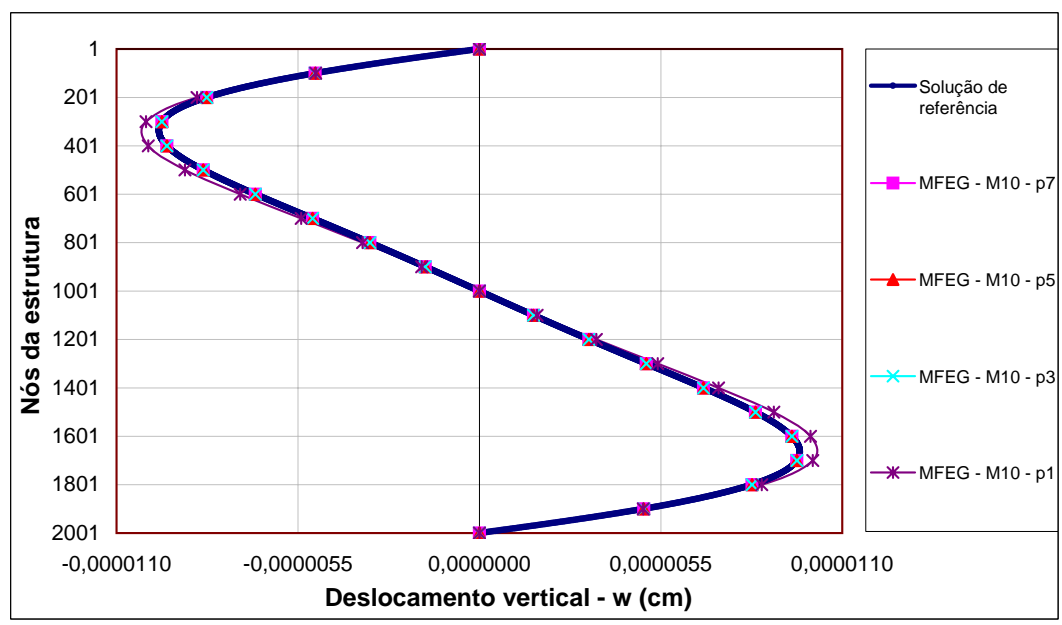

Figura 58: Deslocamentos $w$ - Solução de referência e refino $\mathrm{p}$

O cilindro é curto, de modo que o efeito de flexão não é estritamente localizado em relação às bordas. Observando-se os resultados obtidos é de se notar a variação dos esforços solicitantes. Tal fato é observado mais significativamente nos diagramas dos momentos fletores, onde se percebe a transição com variação exponencial de valores negativos bastante elevados para valores positivos de menor ordem.

Mesmo com as variações nos esforços o método de enriquecimento utilizado se mostrou bastante eficaz. De fato, com uma rede de 10 elementos finitos e refino p de ordem 3 já se consegue resultados bastante satisfatórios.

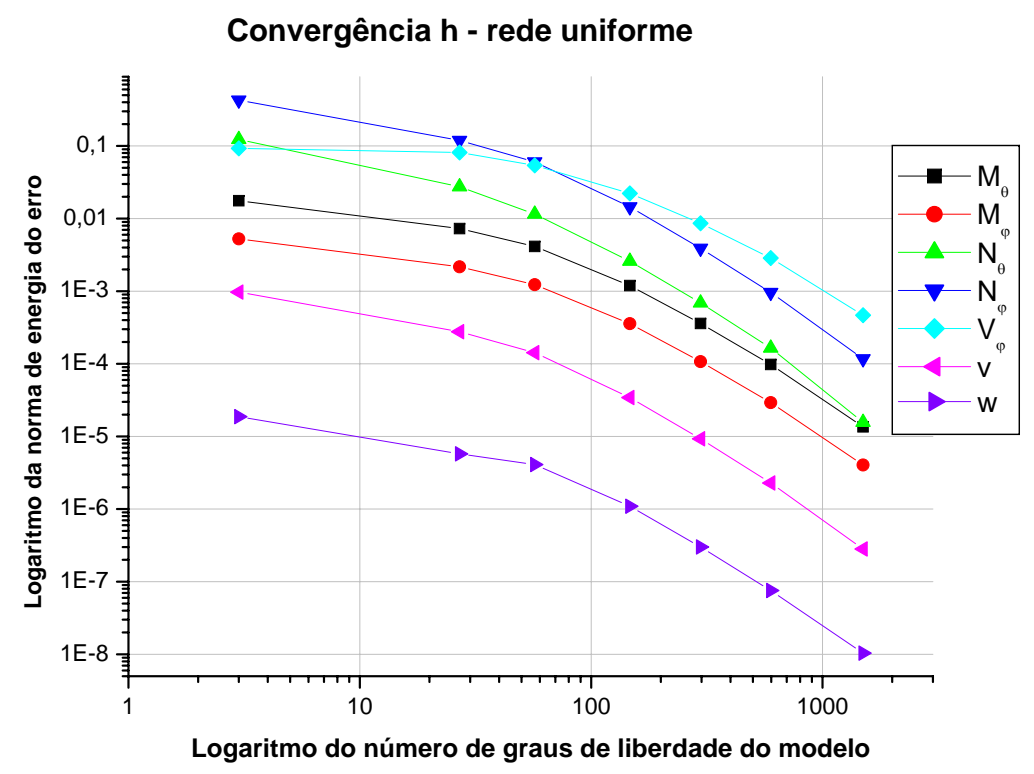

Figura 59: Convergência - refino $h$ da rede

Essa conclusão é reforçada com a visualização dos diagramas de convergência do 


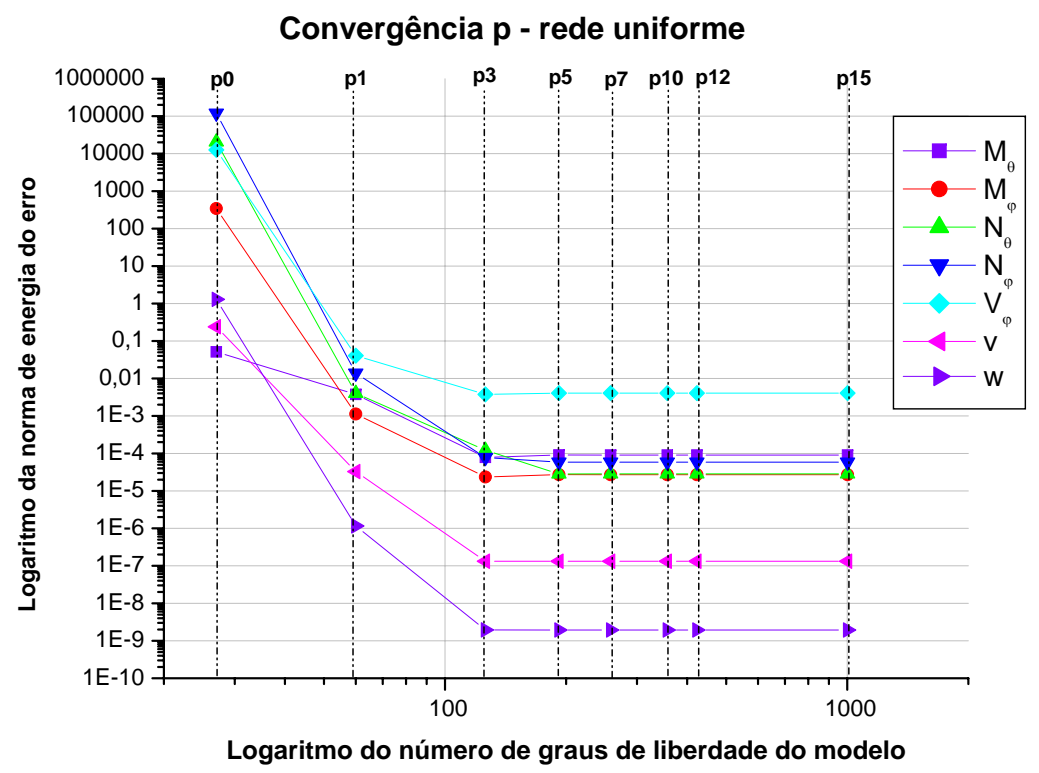

Figura 60: Convergência - refino $p$

refino p. Fica bastante claro que a partir do enriquecimento das funções de aproximação com polinômios de terceira ordem a solução obtida praticamente coincide com a solução de referência do MEF convencional. Entretanto o refino p tem sua efetividade limitada.

Para uma solução ainda mais próxima da solução de referência seria necessário um aumento no número de elementos finitos da rede.

\subsection{Exemplo 04 - Acoplamento cilindro cúpula esférica}

Num primeiro exemplo de superfícies acopladas, propõe-se a análise de uma estrutura de um reservatório composto por uma casca cilíndrica e uma cúpula esférica aberta com a borda superior livre (nó 1 na figura 61) e refino p aplicado a todas as nuvens que compõem o domínio da estrutura.

Para esse problema considerou-se a estrutura submetida a uma força horizontal distribuída de variação linear (hidrostática) e vinculação com o meio externo na base da estrutura (nó 2001 na figura 61) mediante apoio rotulado móvel. As demais características do problema são apresentadas na tabela 4.

Na figura 61 mostra-se um corte esquemático da estrutura analisada e suas dimensões.

Nas análises numéricas do problema, pelo MEF convencional e MEFG, utilizou- 
Tabela 4: Exemplo 04 - Dados de entrada do modelo

\begin{tabular}{c|c}
\hline \hline Espessura constante para toda estrutura & $8 \mathrm{~cm}$ \\
\hline Modulo de elasticidade do material & $2500 \mathrm{kN} / \mathrm{cm}^{2}$ \\
\hline Coeficiente de Poisson & 0,2 \\
\hline Raio de curvatura da superfície esférica & $\sqrt{80} \mathrm{~m}$ \\
\hline Peso especifico do liquido & $10 \mathrm{kN} / \mathrm{m}^{3}$ \\
\hline
\end{tabular}

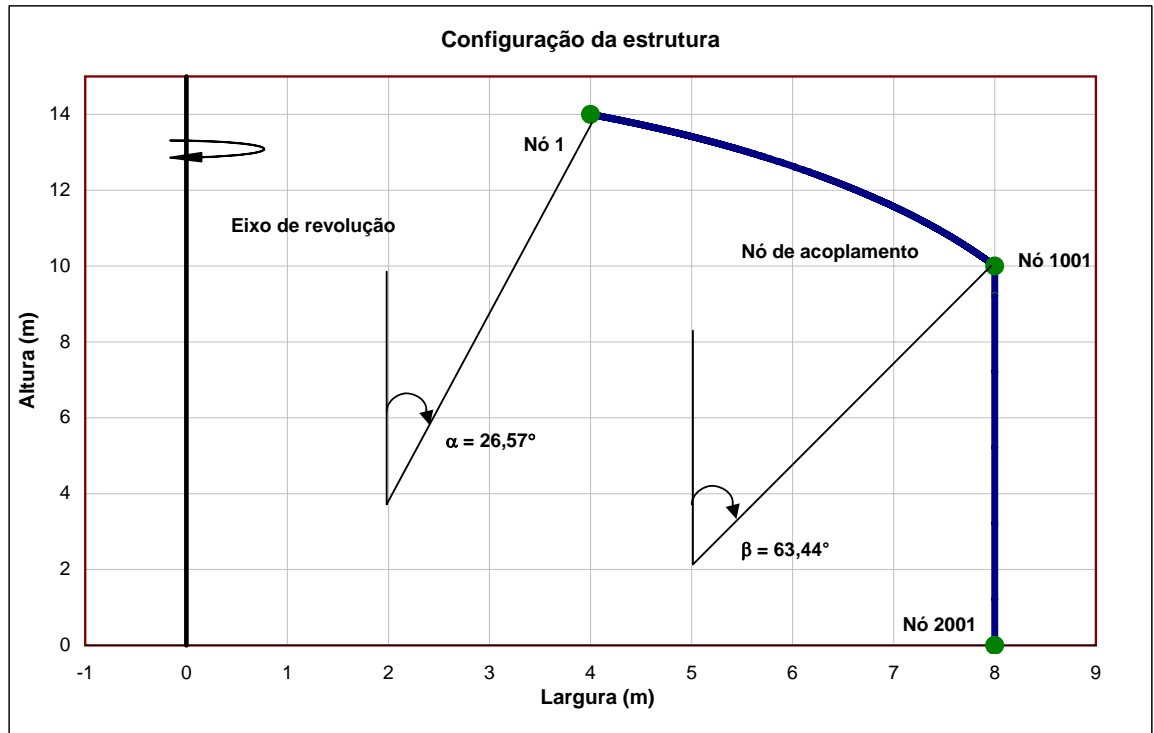

Figura 61: Exemplo 04 - Corte esquemático da estrutura

se de refino h no caso do MEF e de refino p para o MEFG, com o número constante de elementos neste último caso. A análise com o MEF serviu para definir a solução de referência e a curva para análise da convergência.

Em confronto com a solução de referência são avaliadas as respostas obtidas pelo MEFG com diferentes ordens polinomiais de enriquecimento.

Fixou-se uma rede de 20 elementos finitos, todos de igual comprimento, optandose por discretizar cada uma das cascas com 10 elementos finitos.

Os resultados mostrados são aqui na forma de diagramas de momentos fletores $\left(M_{\theta}, M_{\varphi}\right)$, forças normais $\left(N_{\theta}, N_{\varphi}\right)$, força cortante $\left(V_{\varphi}\right)$ e deslocamentos radial e vertical $(v$ e $w)$, segundo a convenção mostrada na figura 10 do capítulo 2.

Observando-se os gráficos apresentados, nota-se que com um pequeno aumento 


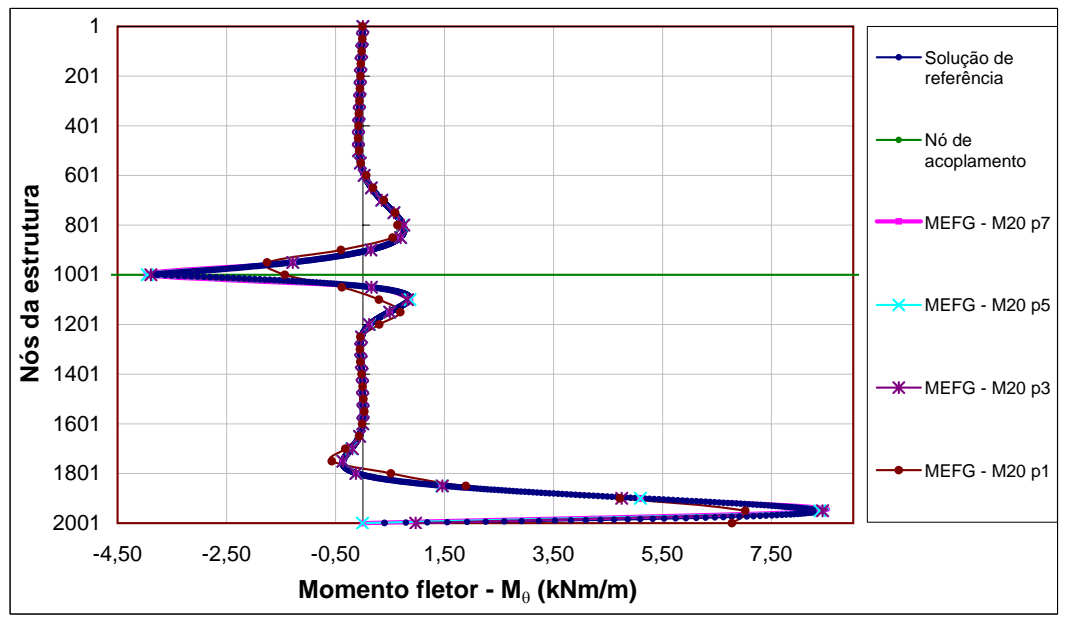

Figura 62: Momentos fletores $M_{\theta}$ - Solução de referência e refino p

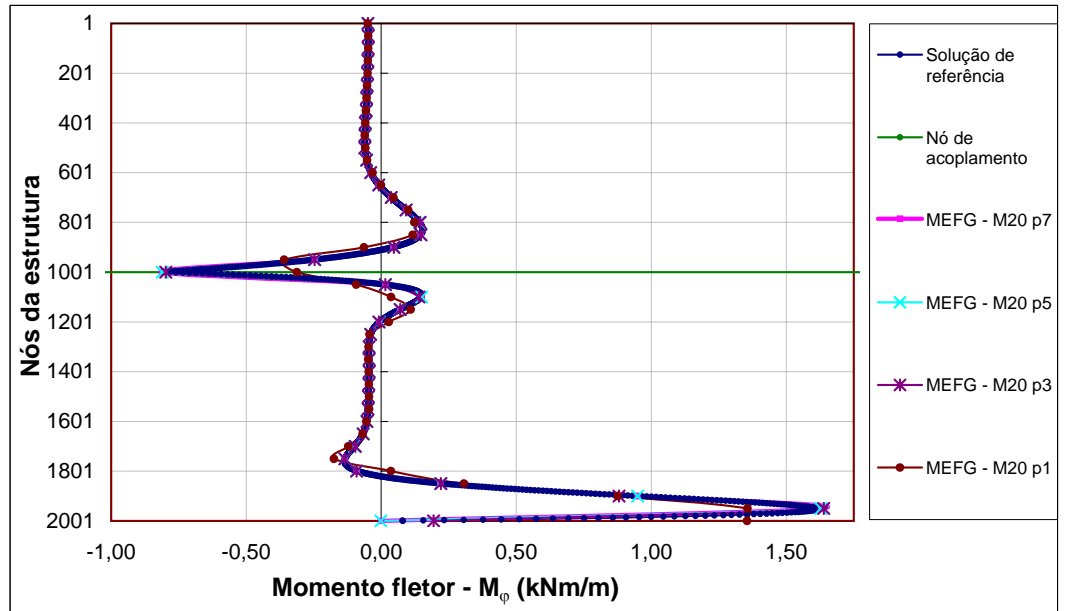

Figura 63: Momentos fletores $M_{\varphi}$ - Solução de referência e refino p

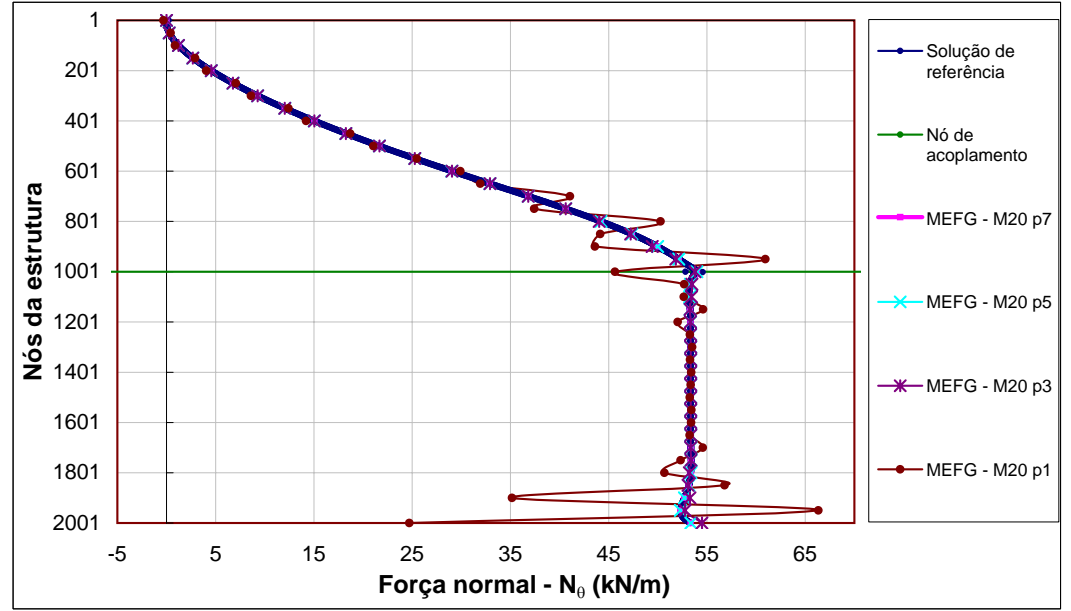

Figura 64: Forças normais $N_{\theta}$ - Solução de referência e refino p 


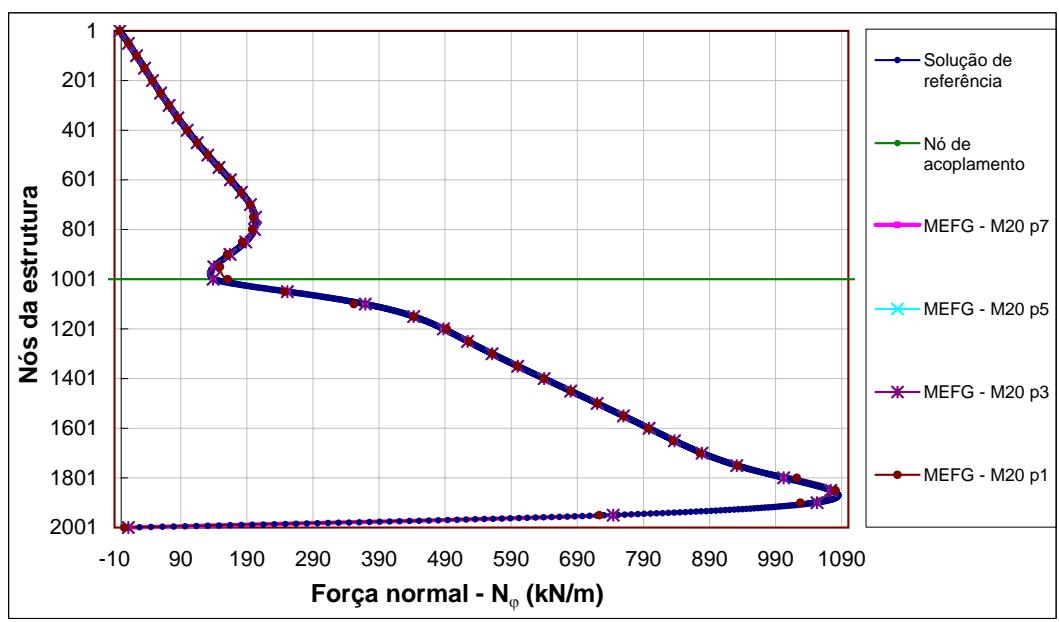

Figura 65: Forças normais $N_{\varphi}$ - Solução de referência e refino p

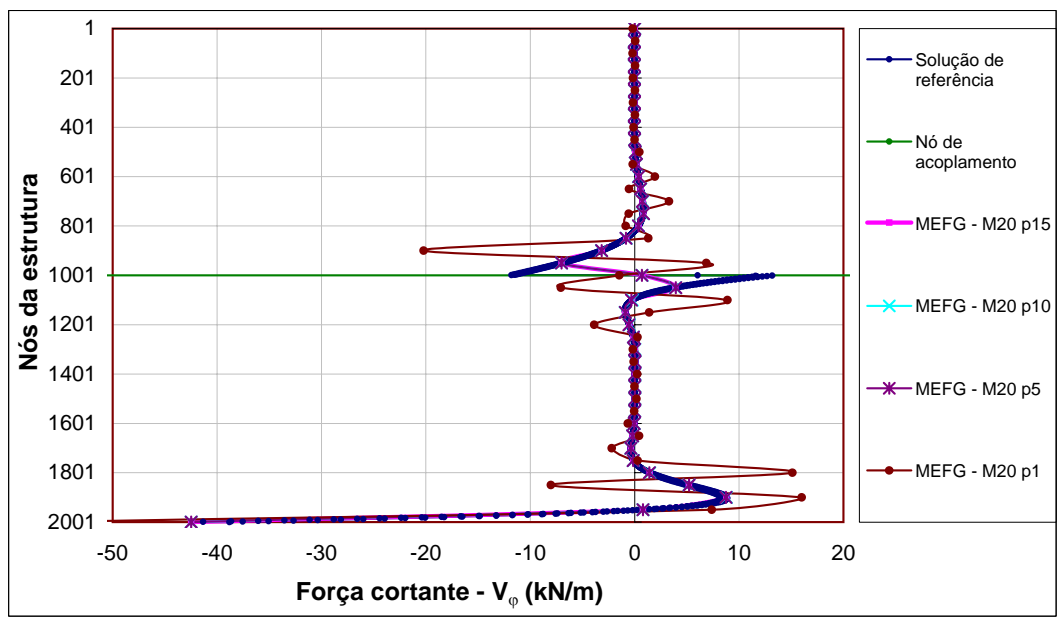

Figura 66: Forças cortantes $V_{\varphi}$ - Solução de referência e refino p

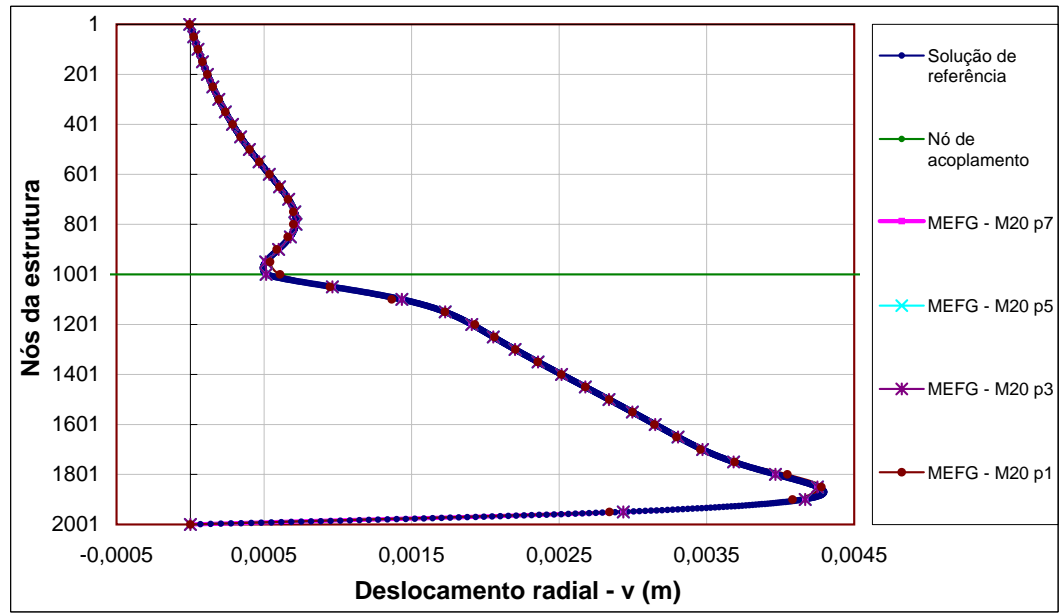

Figura 67: Deslocamentos $v$ - Solução de referência e refino p 


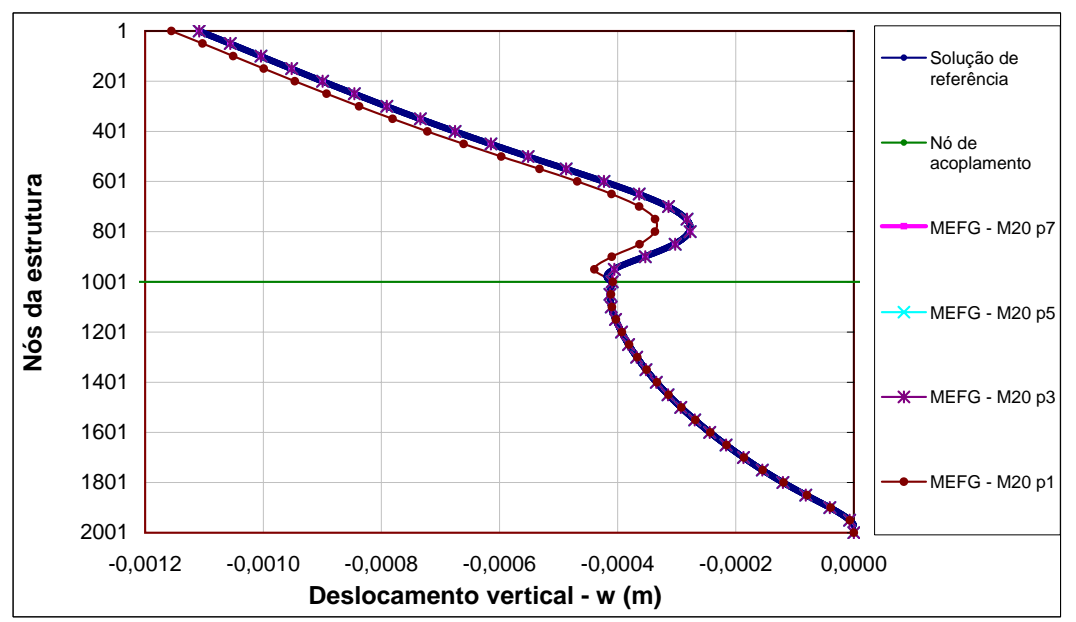

Figura 68: Deslocamentos $w$ - Solução de referência e refino p

na ordem polinomial da função de enriquecimento a solução já passa a ser praticamente coincidente com a solução de referência. A força cortante $\left(V_{\varphi}\right)$ exige refino maior e esse fato é devido à forte perturbação do regime de membrana junto às regiões de vinculação externa e de junção das superfícies.

Uma outra forma de avaliação dos resultados decorre dos gráficos da norma energia do erro em função do número de graus de liberdade do modelo, apresentados nas figuras 69 e 70 .

Rigorosamente, pela figura 70 pode-se concluir que o enriquecimento das funções de aproximação com polinômios de quinta ordem, com a rede de 20 elementos, não traz ganhos significativos a solução do problema. Entretanto, em termos gerais a utilização de um polinômio do terceiro grau como forma de enriquecimento da função de aproximação já foi suficiente para capturar de forma satisfatória a solução do problema.

Como pode se perceber, o refino p propicia um ganho imediato em relação à taxa de convergência, porém limitado aos primeiros graus de enriquecimento, a partir dos quais, o ganho de precisão passar a ser muito pequeno em relação ao custo computacional de resolução do problema. Isso indica que para melhorar ainda mais a qualidade dos resultados seria conveniente combinar um refino da rede de elementos, o que é absolutamente consistente com a conhecida noção de maior eficiência do refino h-p. 


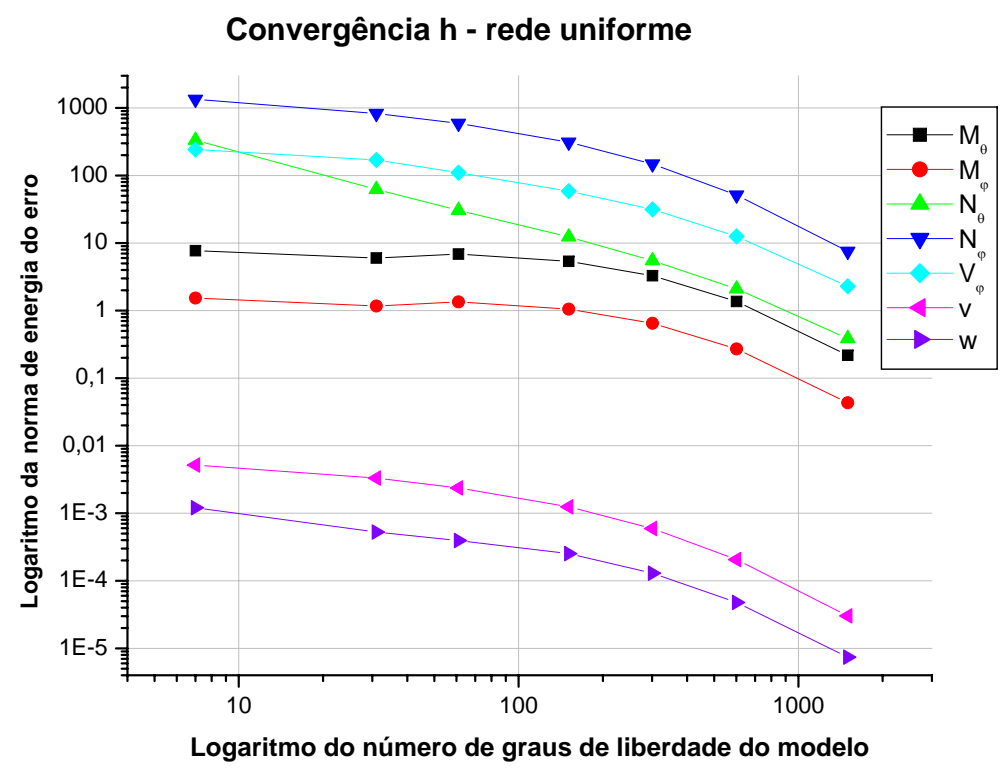

Figura 69: Convergência - refino $h$ da rede

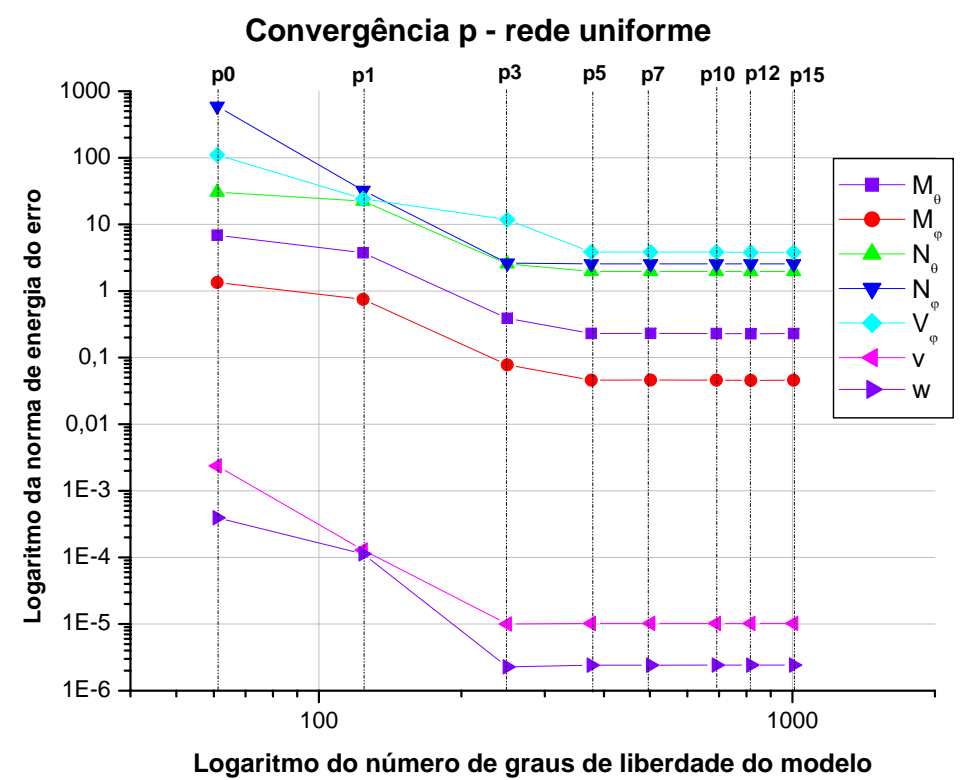

Figura 70: Convergência - refino $p$

\subsection{Exemplo 05 - Acoplamento tronco de cone placa circular}

Nesta aplicação, objetivou-se explorar mais uma vez a capacidade do elemento finito utilizado em adaptar-se a formas quaisquer de superfícies.

Escolheu-se uma casca composta por uma superfície tronco-cônica associada a uma placa circular. O conjunto está sujeito a pressão interna uniforme e vinculado ao 
meio externo através de um apoio rotulado móvel na base da estrutura (nó 2001). As demais características do problema estão expostas na tabela 5.

Tabela 5: Exemplo 05 - Dados de entrada do modelo

\begin{tabular}{c|c}
\hline \hline Espessura constante para toda estrutura & $2 \mathrm{~mm}$ \\
\hline Modulo de elasticidade do material & $20500 \mathrm{kN} / \mathrm{cm}^{2}$ \\
\hline Coeficiente de Poisson & 0,3 \\
\hline Pressão interna uniforme & $0,01 \mathrm{kN} / \mathrm{cm}^{2}$ \\
\hline
\end{tabular}

Na figura 71 mostra-se um corte esquemático da estrutura analisada e suas dimensões.

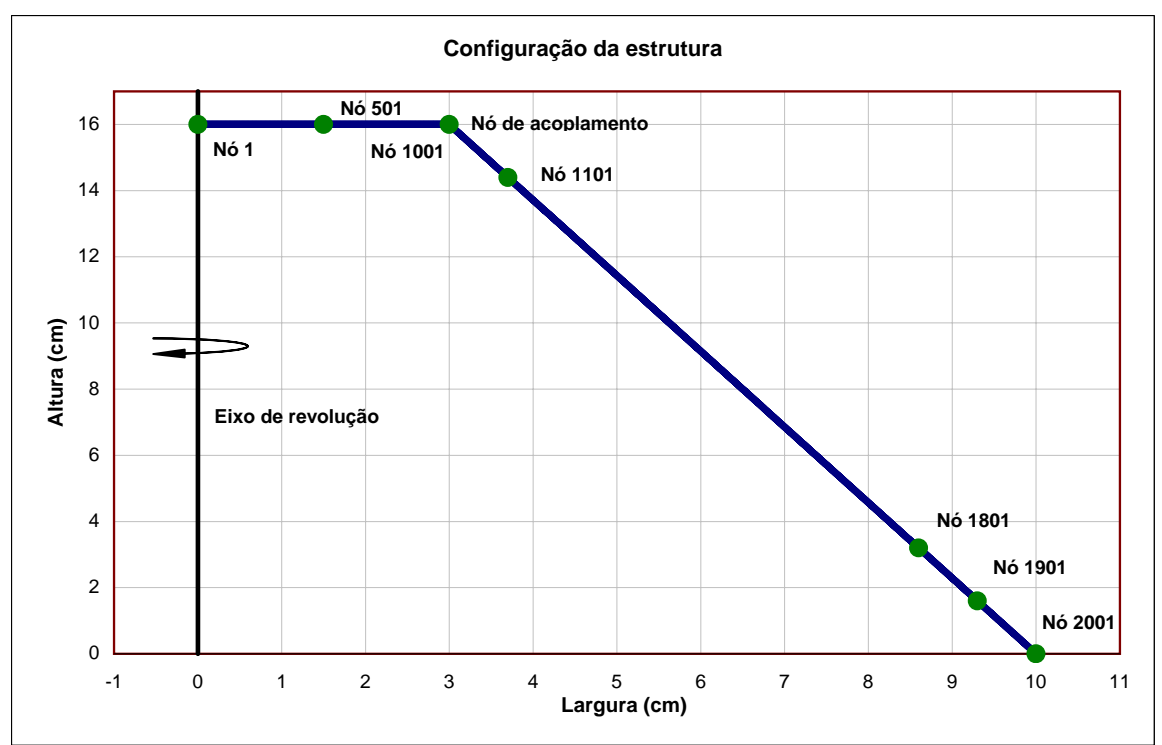

Figura 71: Exemplo 05 - Corte esquemático da estrutura

Da mesma forma que no exemplo anterior, adotou-se uma discretização formada por 10 elementos finitos, de mesmo tamanho em cada umas das superfícies. Efetuou-se um mesmo tipo de análise, explorando-se refinos h e p da rede para avaliação da convergência dos resultados.

A escolha dos nós a terem sua solução enriquecida foi feita de acordo com a perturbação do regime de membrana sofrido pela estrutura. Nesse sentido, além das regiões de acoplamento e vinculação das casca tronco-cônica, toda a placa foi enriquecida. Foram aplicadas funções de enriquecimento nos seguintes nós: 1 a 1101 e 1801 a 2001. Vale lembrar que a base de referência para montagem da rede a ser enriquecida segue a mesma numeração da rede montada para solução de referência que esta baseada no MEF convencional. 
Devido à dificuldade de representação dos resultados de esforços e deslocamentos na estrutura como um todo, os gráficos são apresentados para cada uma partes, a fim de facilitar sua visualização. Os principais resultados estão reunidos nas figuras 72 a 85 .

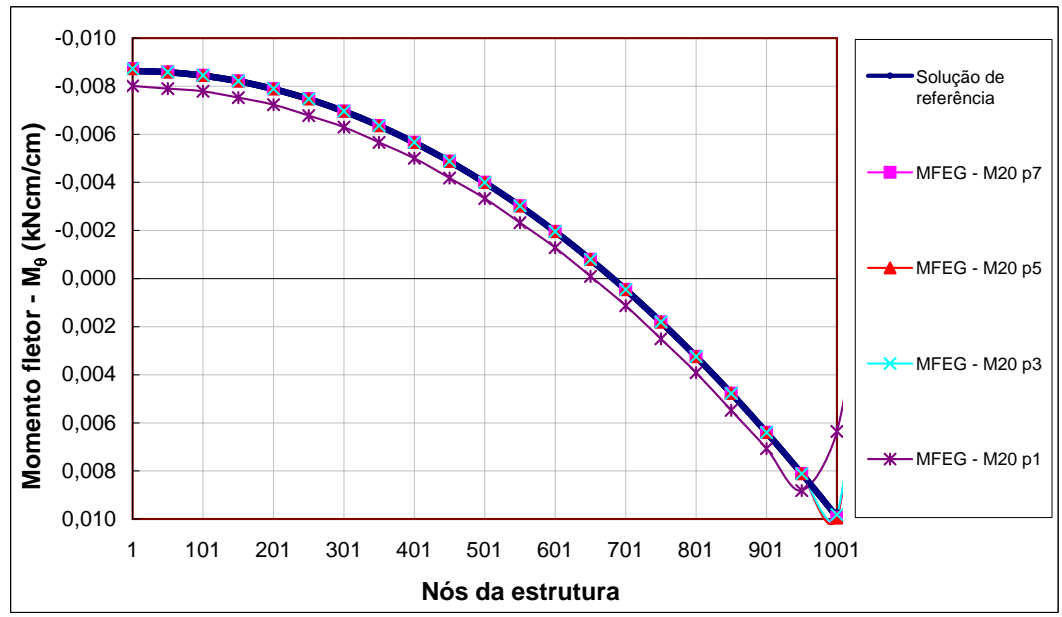

Figura 72: Momentos fletores $M_{\theta}$ - Solução de referência e refino p - Placa

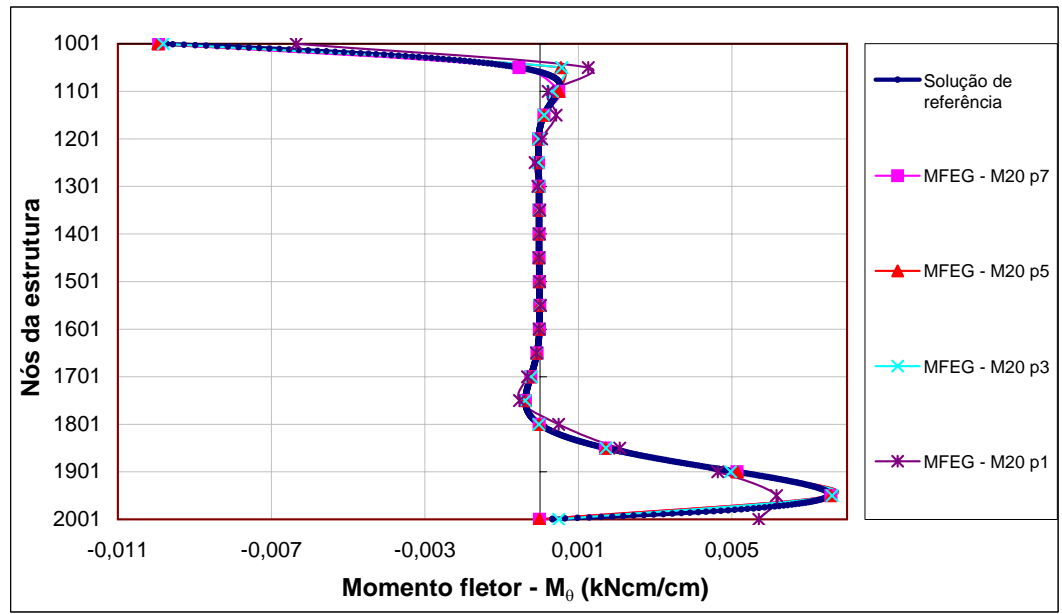

Figura 73: Momentos fletores $M_{\theta}$ - Solução de referência e refino p - Tronco de cone

A avaliação dos resultados se completa com a análise da convergência, com refino $h$ e refino $p$ da rede de elementos, obtidos com o código computacional desenvolvido.

Assim como nos exemplos anteriores, nota-se que para o refino h, a taxa inicial de convergência com o refino da rede é pequena. Já o mesmo não ocorre para o caso analisado através do refino p. Para tal opção, percebe-se que o ganho na taxa de convergência é alto nos primeiros pontos mostrados nas curvas do diagrama de erro.

Em tese o refino p é mais eficiente em relação ao refino h enquanto a parte regular da solução for preponderante. O posterior aumento da ordem polinomial de enriquecimento, entretanto, não traz ganhos significativos para a solução do problema. Esse fato 


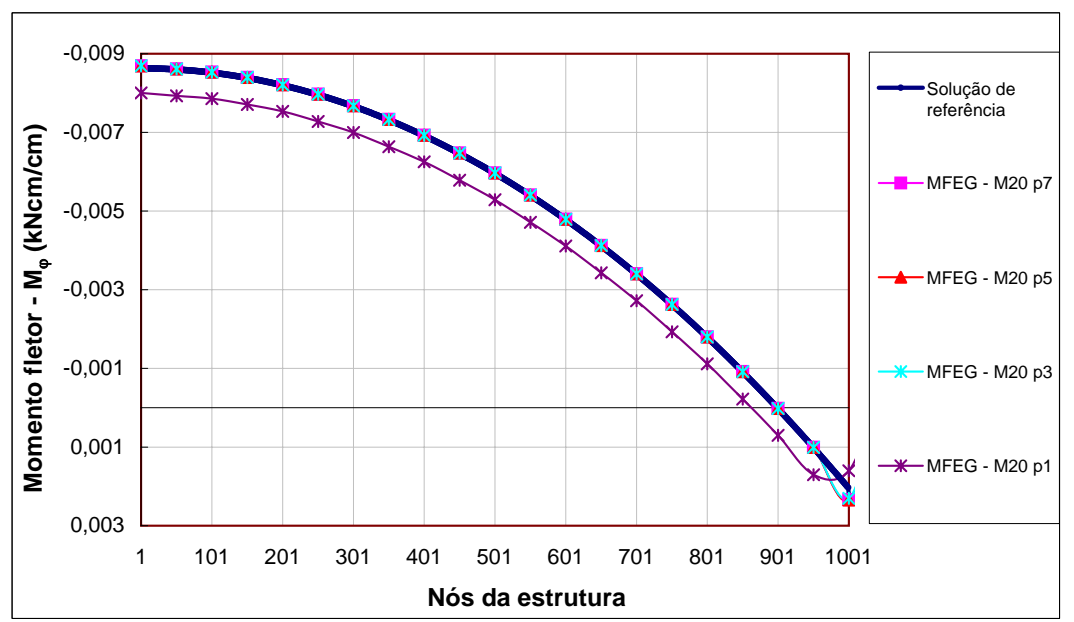

Figura 74: Momentos fletores $M_{\varphi}$ - Solução de referência e refino p - Placa

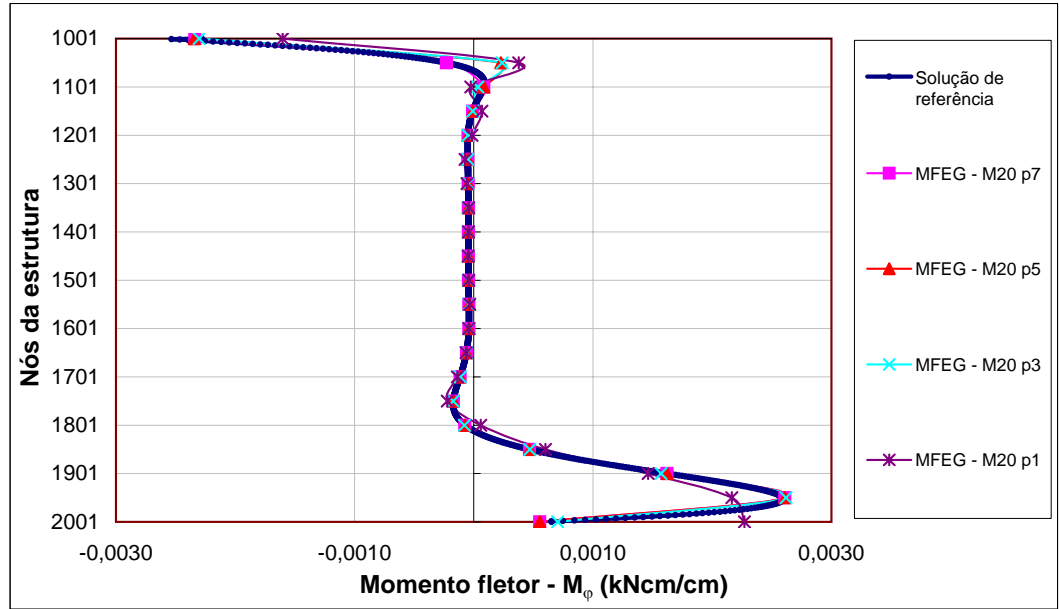

Figura 75: Momentos fletores $M_{\varphi}$ - Solução de referência e refino p - Tronco de cone

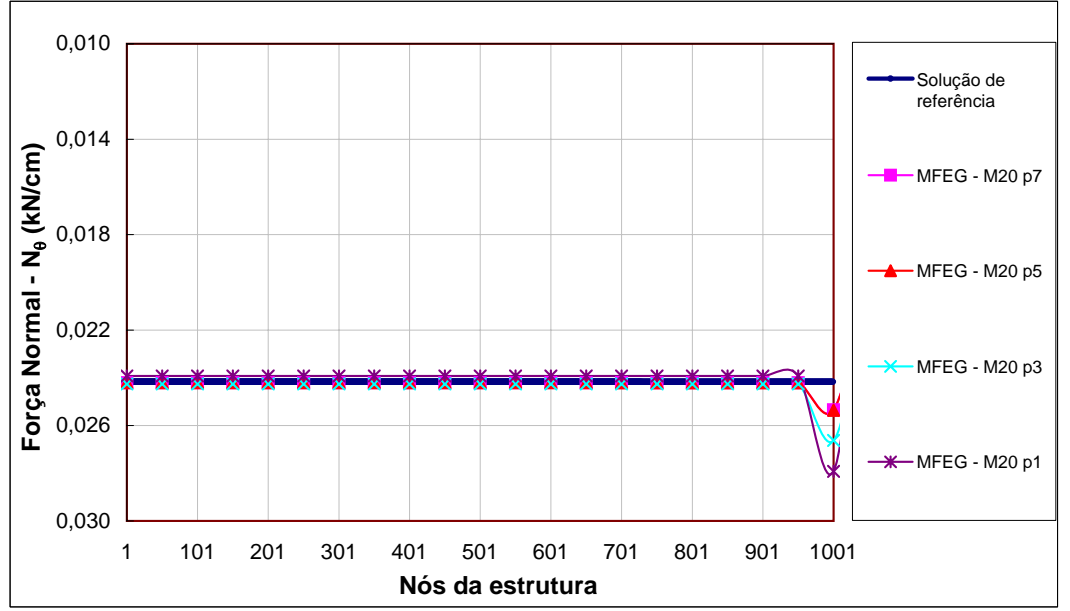

Figura 76: Forças normais $N_{\theta}$ - Solução de referência e refino p - Placa 


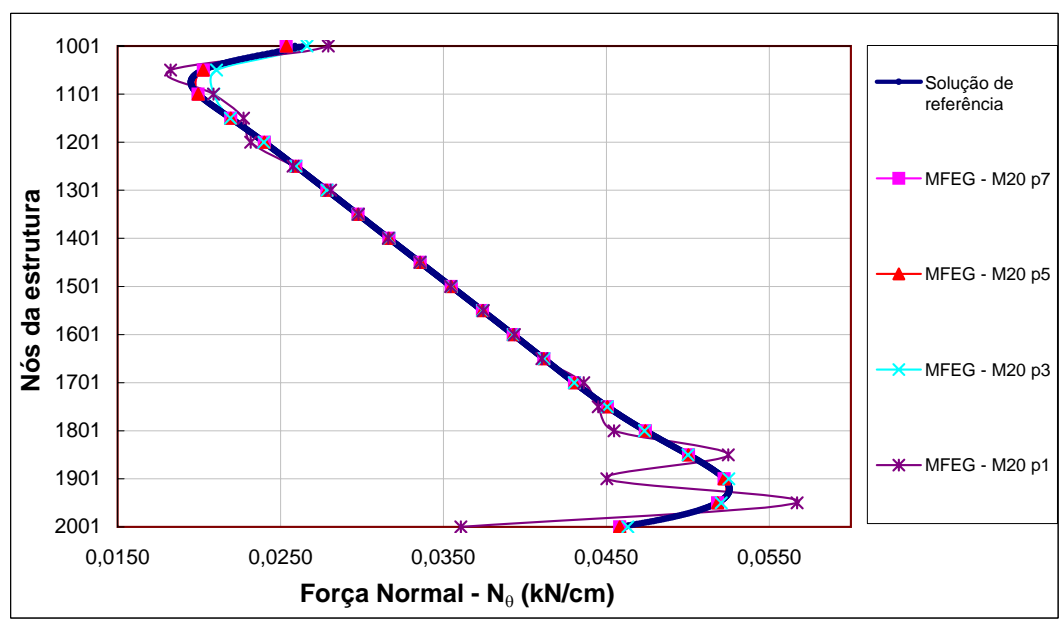

Figura 77: Forças normais $N_{\theta}$ - Solução de referência e refino p - Tronco de cone

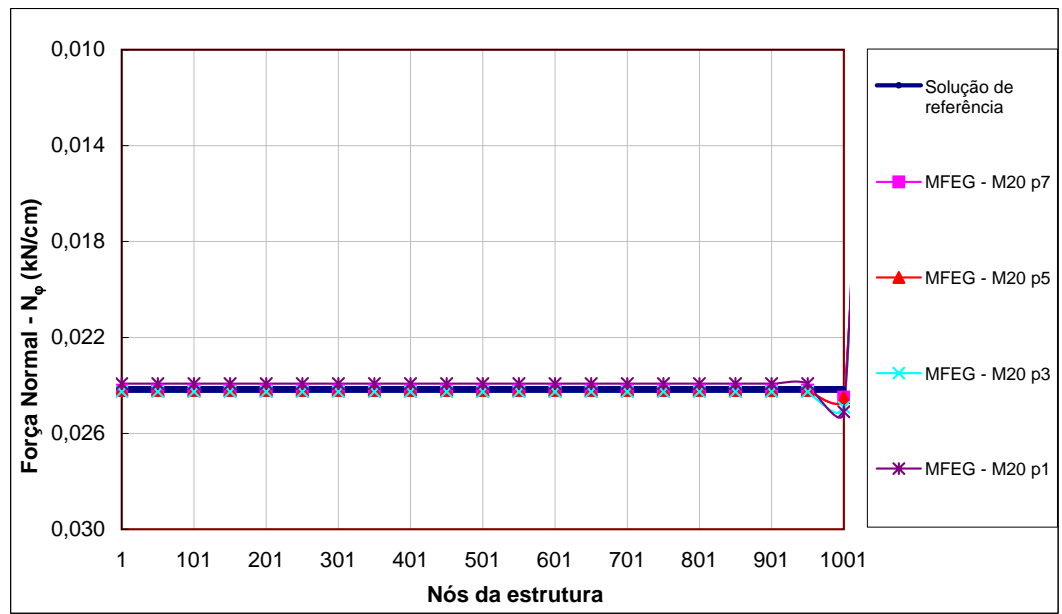

Figura 78: Forças normais $N_{\varphi}$ - Solução de referência e refino p - Placa

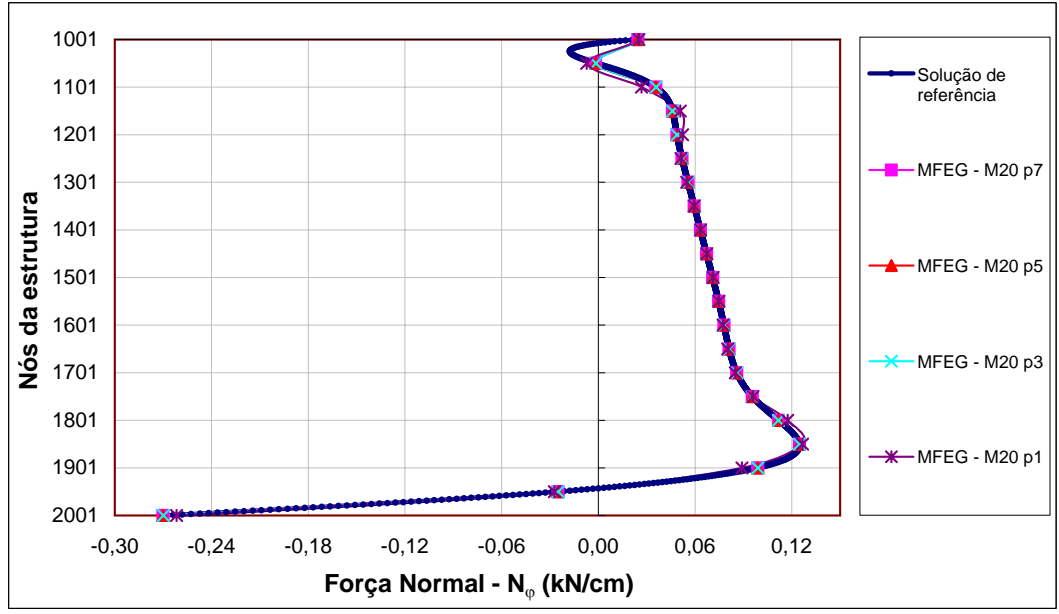

Figura 79: Forças normais $N_{\varphi}$ - Solução de referência e refino p - Tronco de cone 


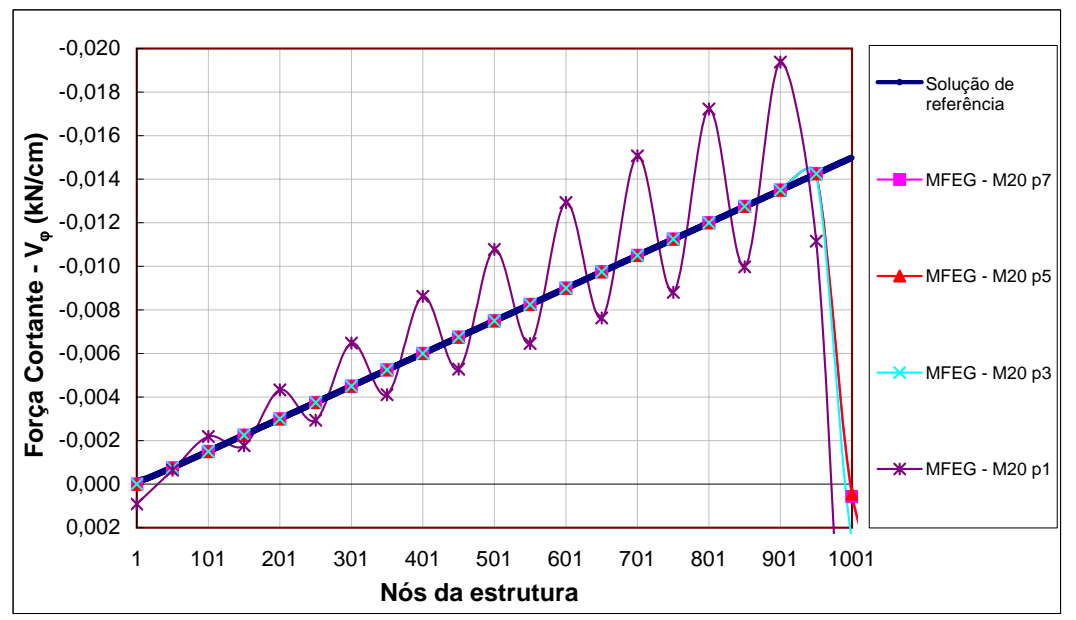

Figura 80: Forças Cortantes $V_{\varphi}$ - Solução de referência e refino p - Placa

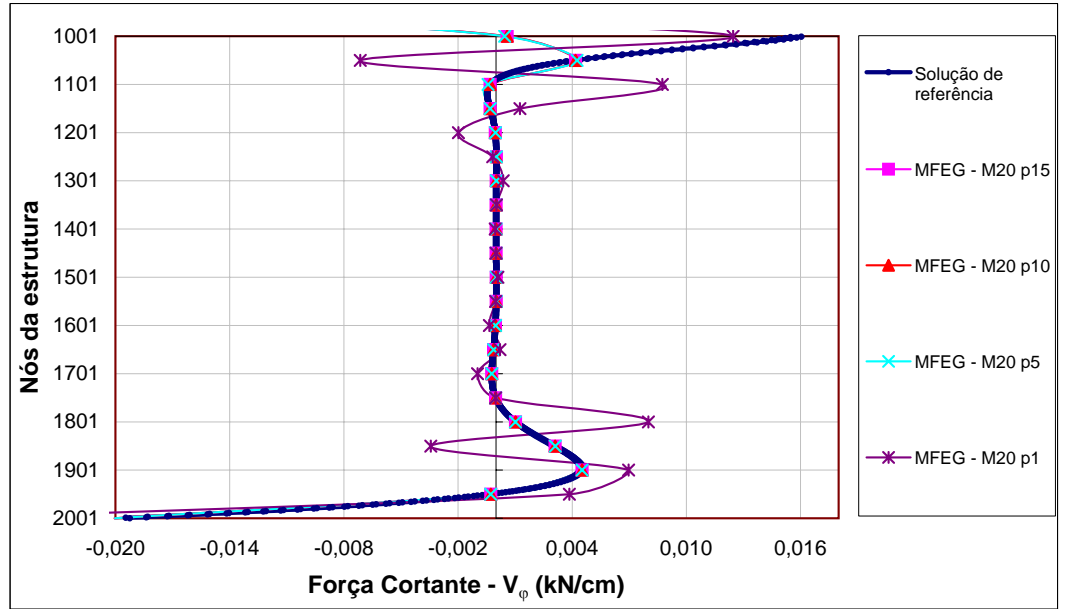

Figura 81: Forças cortantes $V_{\varphi}$ - Solução de referência e refino p - Tronco de cone

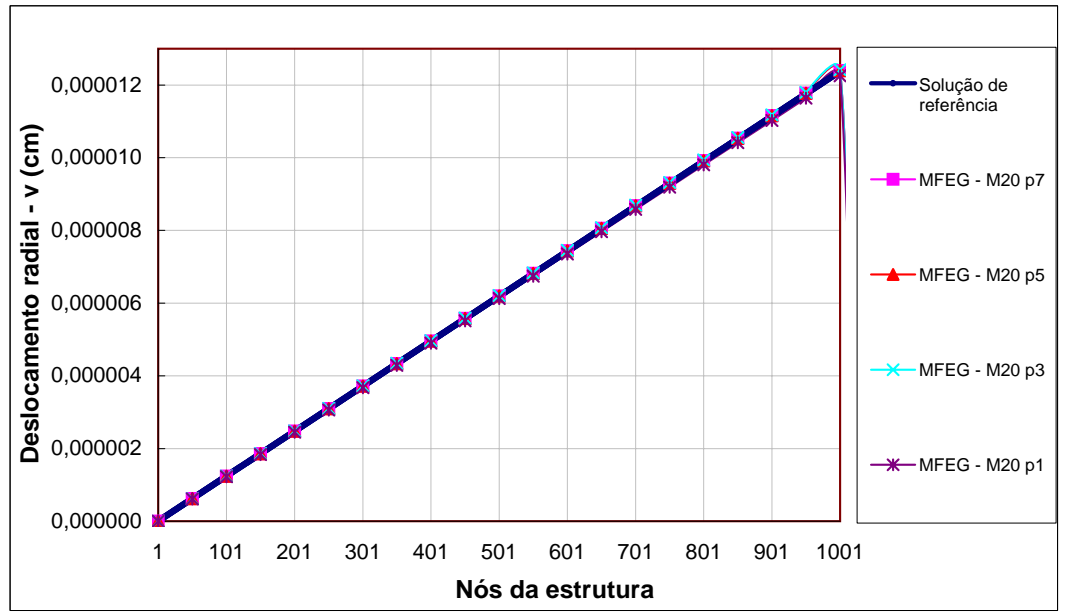

Figura 82: Deslocamentos $v$ - Solução de referência e refino p - Placa 


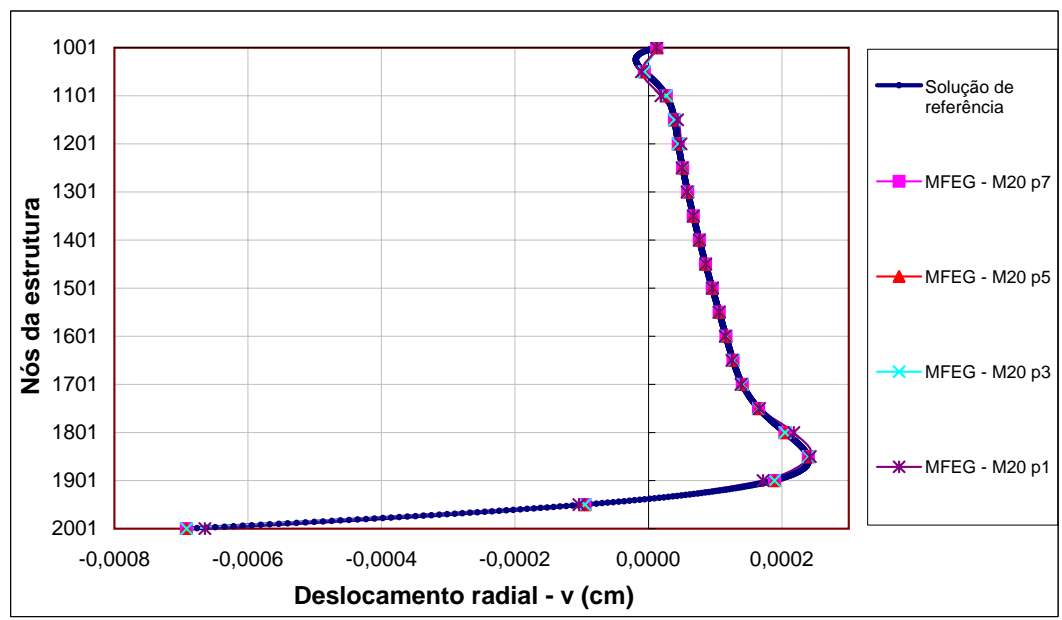

Figura 83: Deslocamentos $v$ - Solução de referência e refino p - Tronco de cone

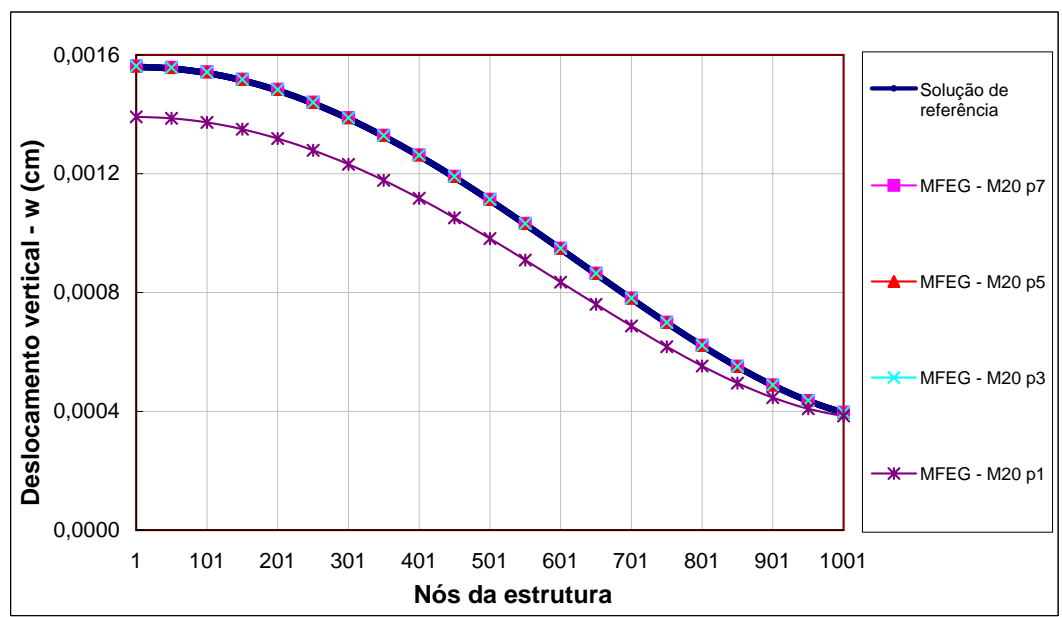

Figura 84: Deslocamentos $w$ - Solução de referência e refino p - Placa

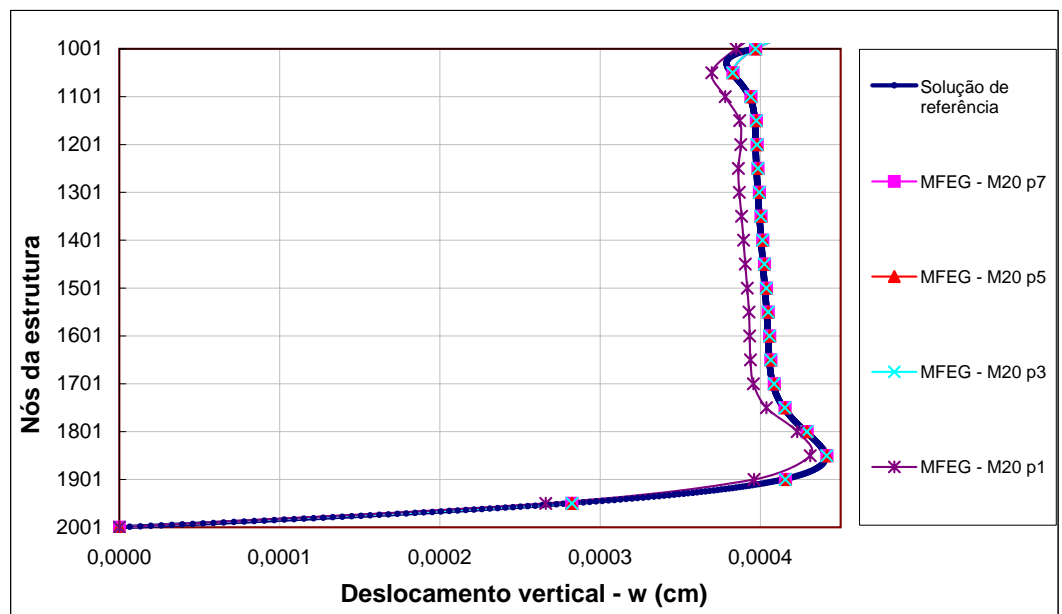

Figura 85: Deslocamentos $w$ - Solução de referência e refino p - Tronco de cone 


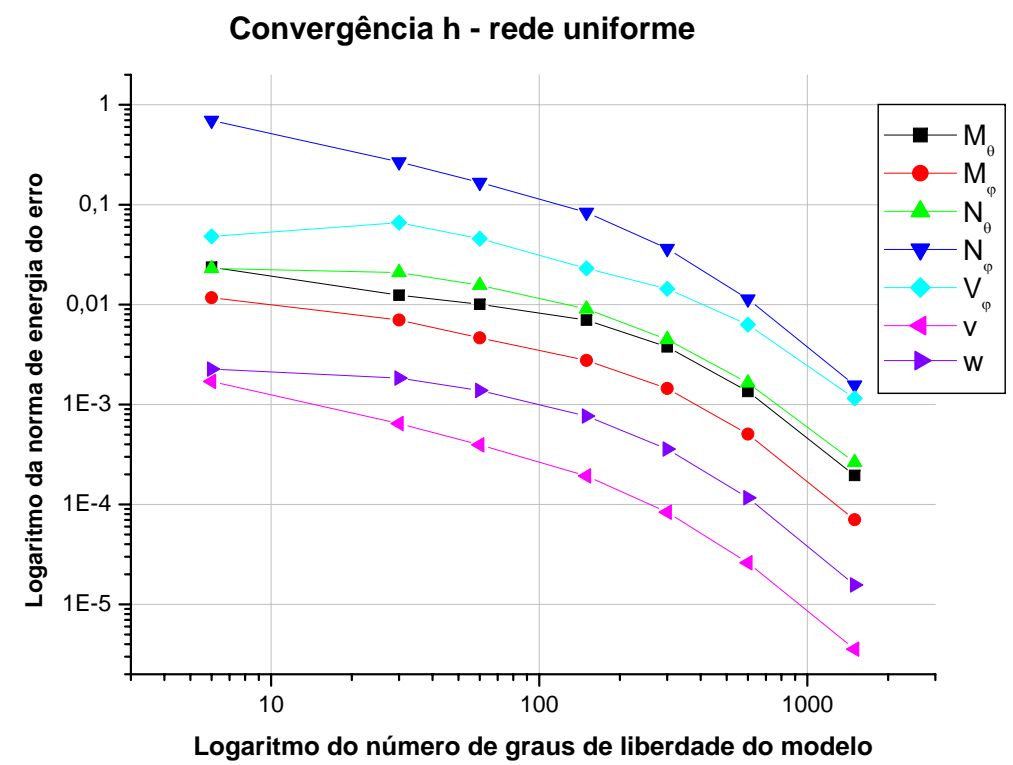

Figura 86: Convergência - refino $h$ da rede

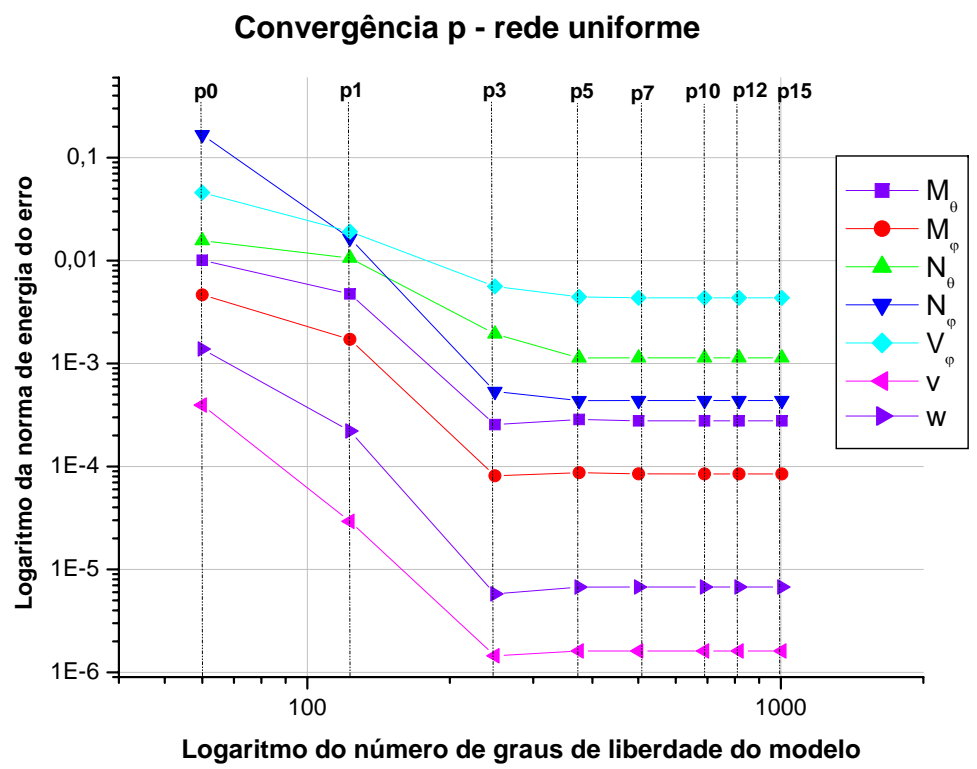

Figura 87: Convergência - refino $p$

pode ser facilmente visualizado no gráfico de erros relativos, a partir do ponto onde a curva se torna uma reta constante; isso significa dizer que independentemente do aumento da ordem polinomial o erro cometido passa a ser praticamente invariável. Assim, apenas o refino p não é suficiente para convergência contínua dos resultados, para tal 
faz-se necessário refinar também a rede de elementos finitos.

\subsection{Exemplo 06 - Superfície hiperbólica}

Neste exemplo de aplicação o objetivo é o de considerar uma superfície com formato diferenciado, para isso escolhendo-se um hiperbolóide de revolução, com características geométricas, físicas e de carregamento, mostradas na figura 88 e na tabela 6 . Para o estudo dessa aplicação utilizou-se do refino p sobre todas as nuvens que cobrem o domínio do problema.

Tabela 6: Exemplo 06 - Dados de entrada do modelo

\begin{tabular}{c|c}
\hline \hline Espessura constante para toda estrutura & $6 \mathrm{~cm}$ \\
\hline Modulo de elasticidade do material & $20500 \mathrm{kN} / \mathrm{cm}^{2}$ \\
\hline Coeficiente de Poisson & 0,2 \\
\hline Peso específico do líquido & $8 \mathrm{kN} / \mathrm{m}^{3}$ \\
\hline
\end{tabular}

Na figura 88, mostra-se um corte esquemático da estrutura analisada e suas dimensões. A vinculação com o meio externo é mediante engaste fixo da base (nó 2001 na figura 88 ) e bordo superior livre (nó 1 na figura 88). O reservatório esta completamente cheio, logo a pressão exercida pelo fluido armazenado atua desde o nó 1 até o nó 2001.

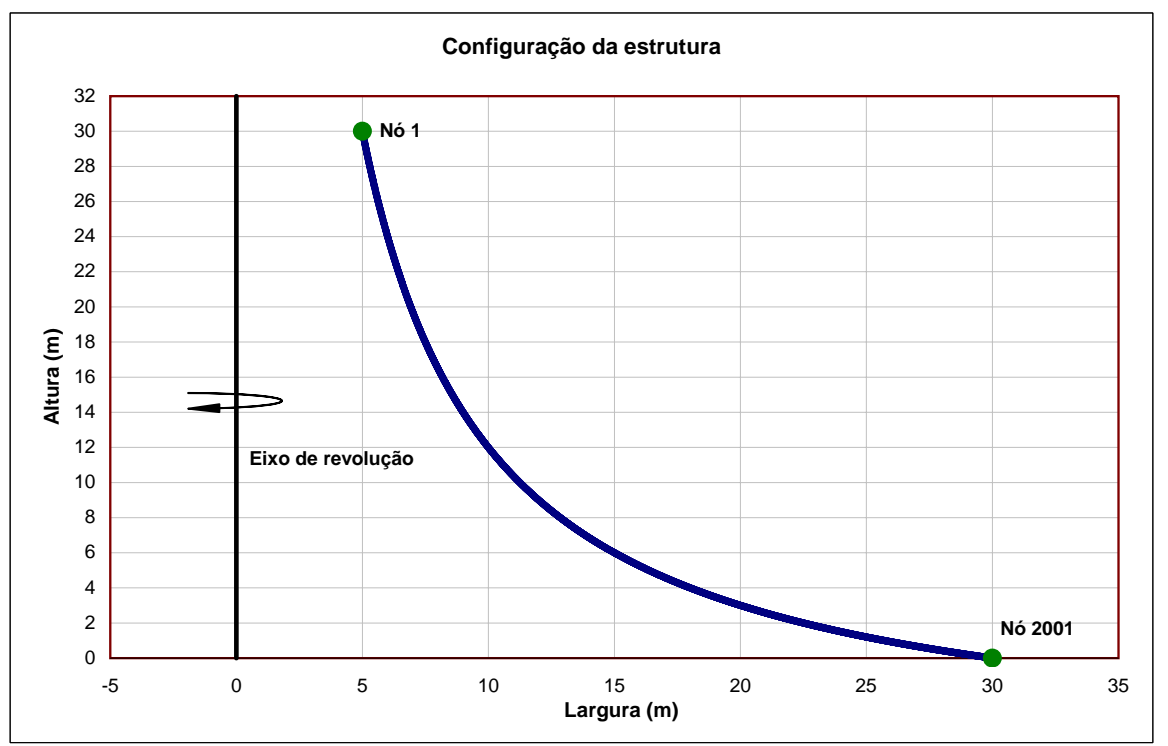

Figura 88: Exemplo 06 - Corte esquemático da estrutura

A discretização é composta por 20 elementos finitos sendo o refino p aplicado a todos os nós. 


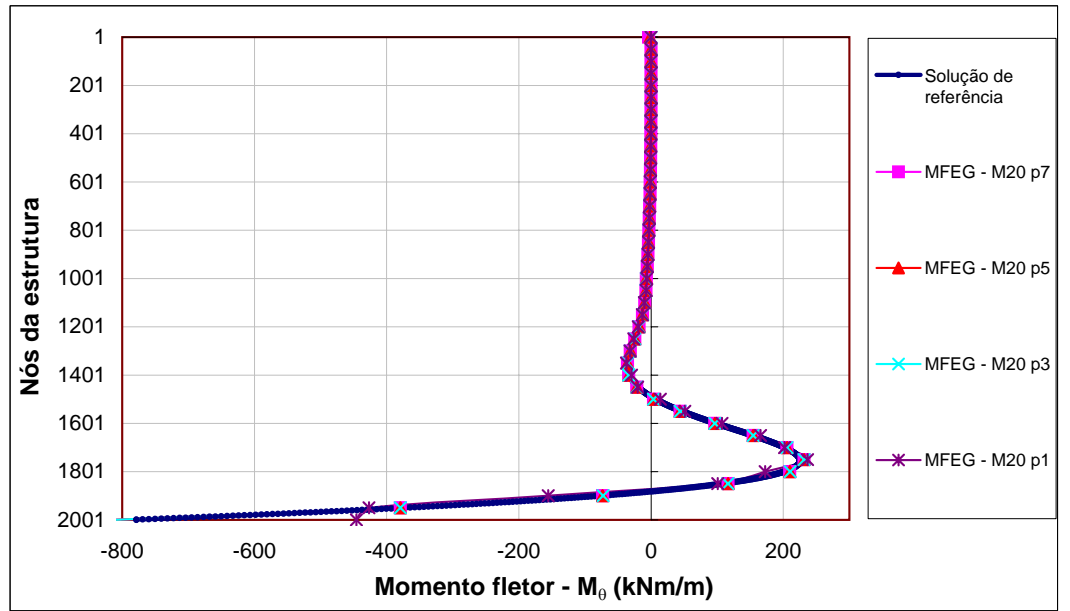

Figura 89: Momentos fletores $M_{\theta}$ - Solução de referência e refino p

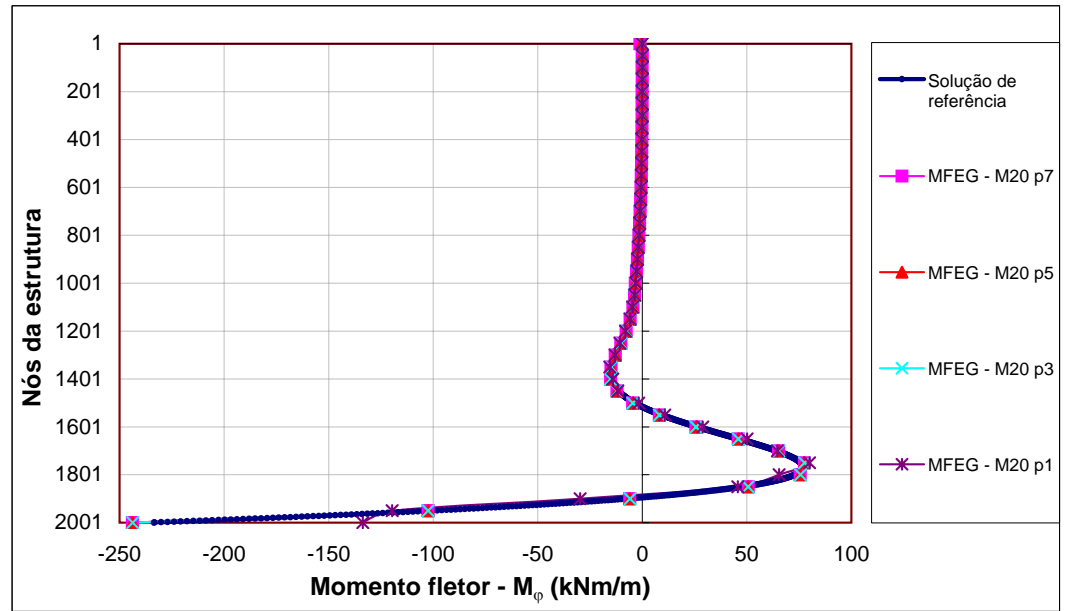

Figura 90: Momentos fletores $M_{\varphi}$ - Solução de referência e refino p

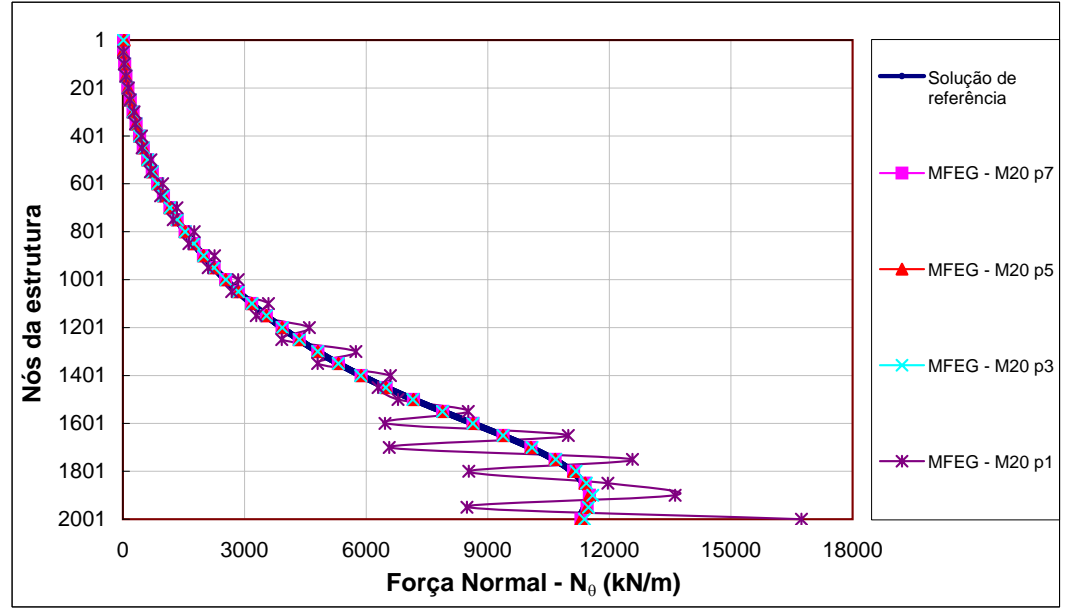

Figura 91: Forças normais $N_{\theta}$ - Solução de referência e refino p 


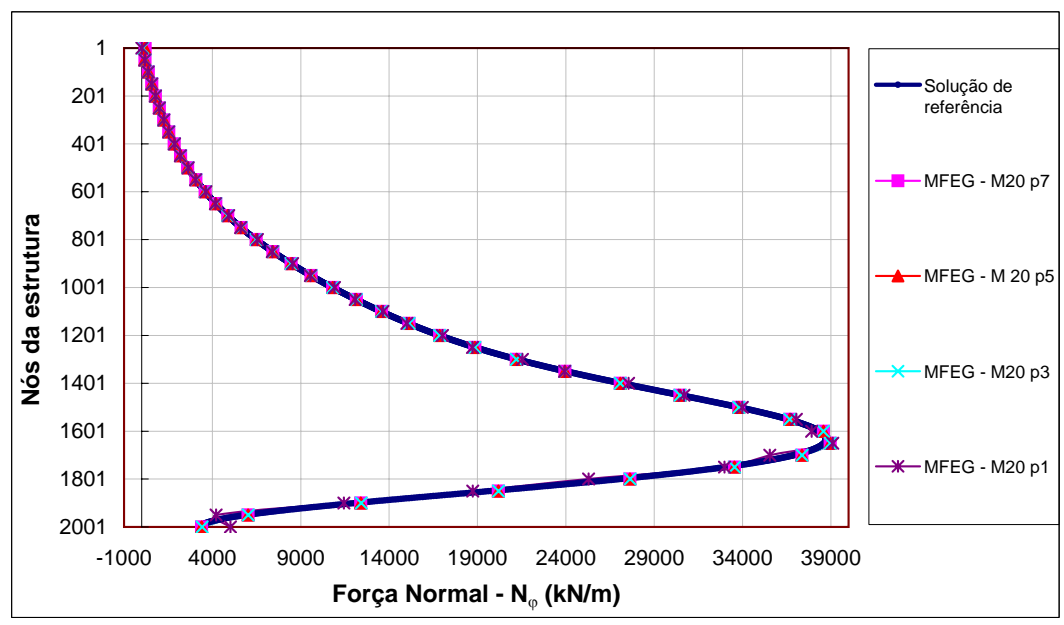

Figura 92: Forças normais $N_{\varphi}$ - Solução de referência e refino p

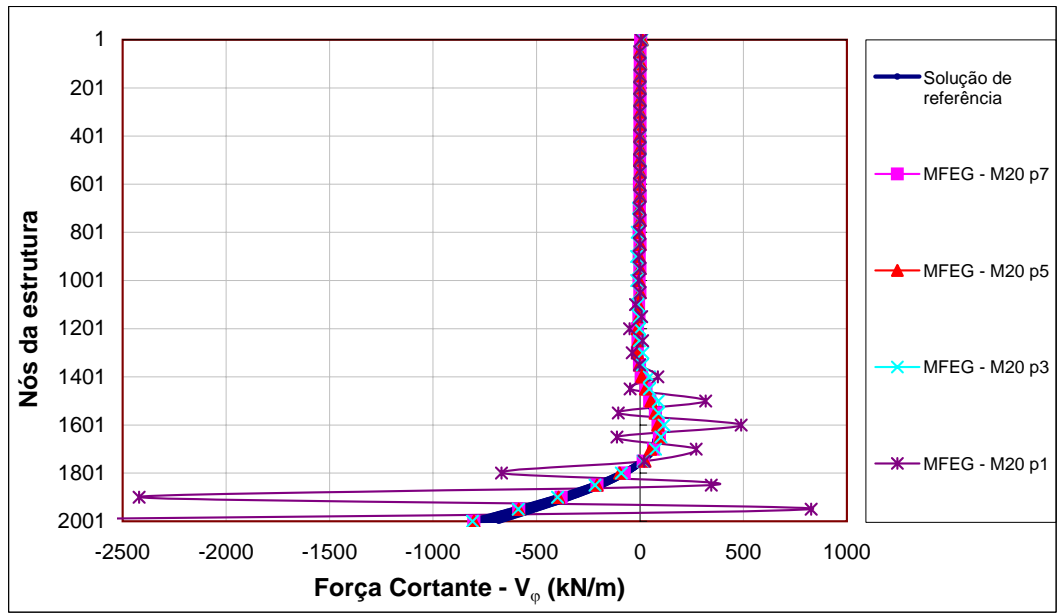

Figura 93: Forças cortantes $V_{\varphi}$ - Solução de referência e refino p

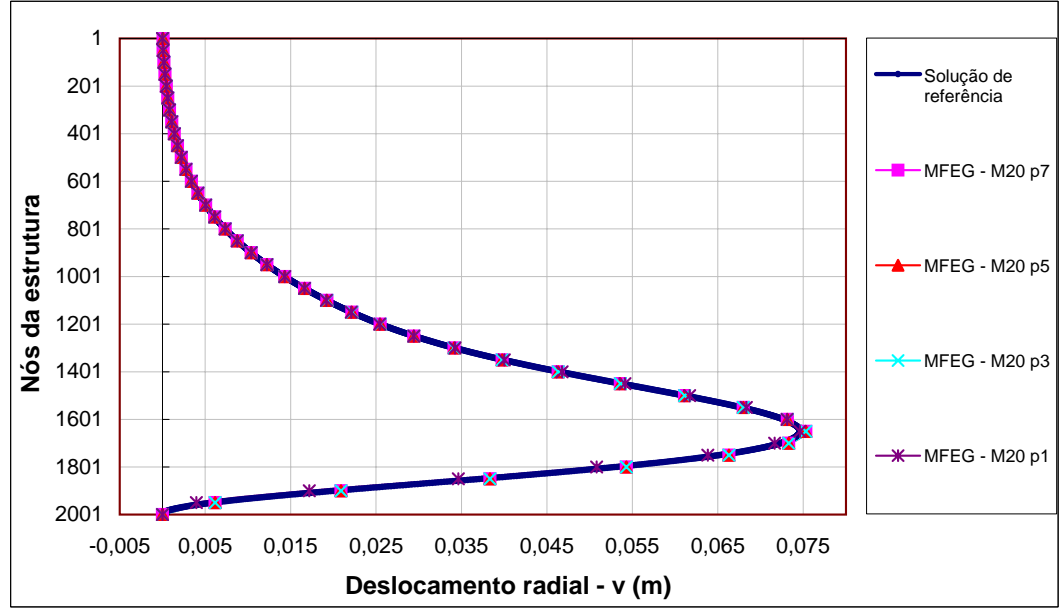

Figura 94: Deslocamentos $v$ - Solução de referência e refino p 


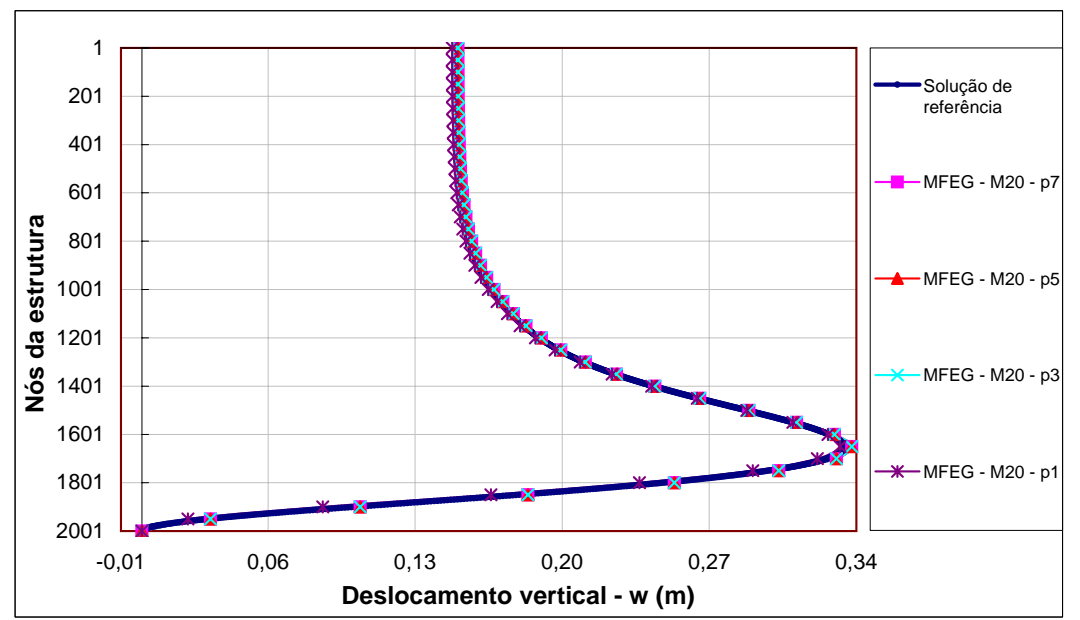

Figura 95: Deslocamentos $w$ - Solução de referência e refino p

Assim como nos exemplos das seções 6.2 a 6.6 ficou evidente a eficiência do método, visto que o refino p com funções de enriquecimento polinomial de terceira ordem já foi suficiente para atingir resultados bastante satisfatórios.

A análise da convergência dos resultados se completa, com os gráficos de erros apresentados nas figuras 96 e 97.

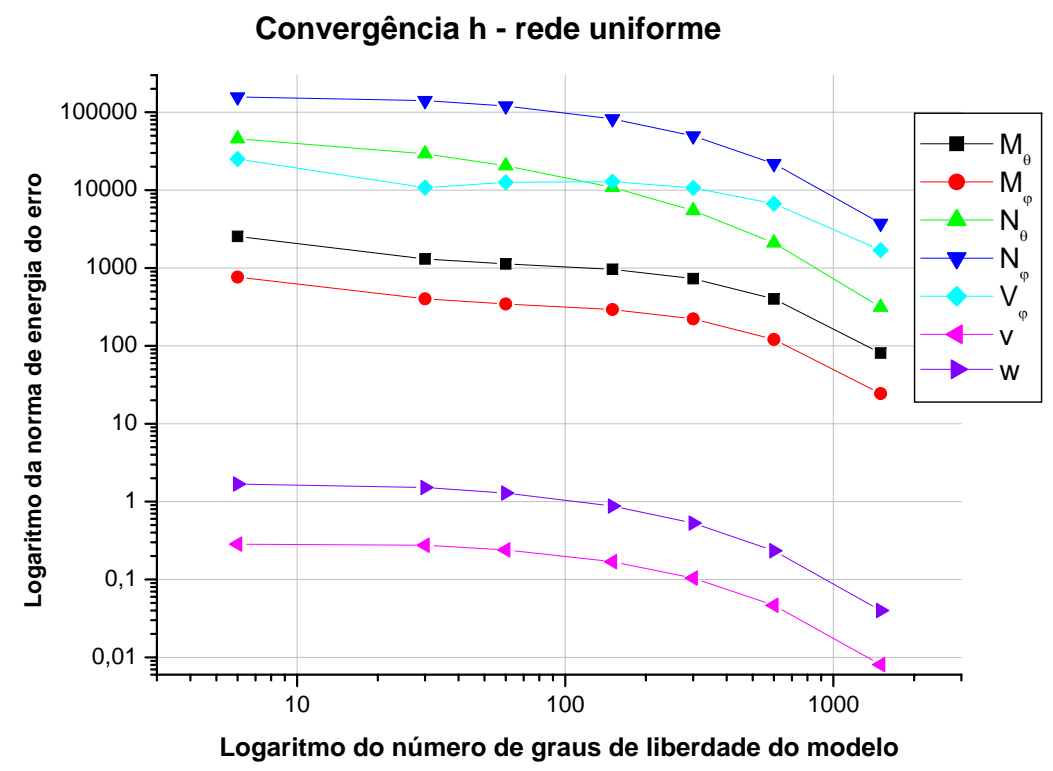

Figura 96: Convergência - refino $h$ da rede

A convergência para solução de referência manteve padrão similar aos dos exem- 


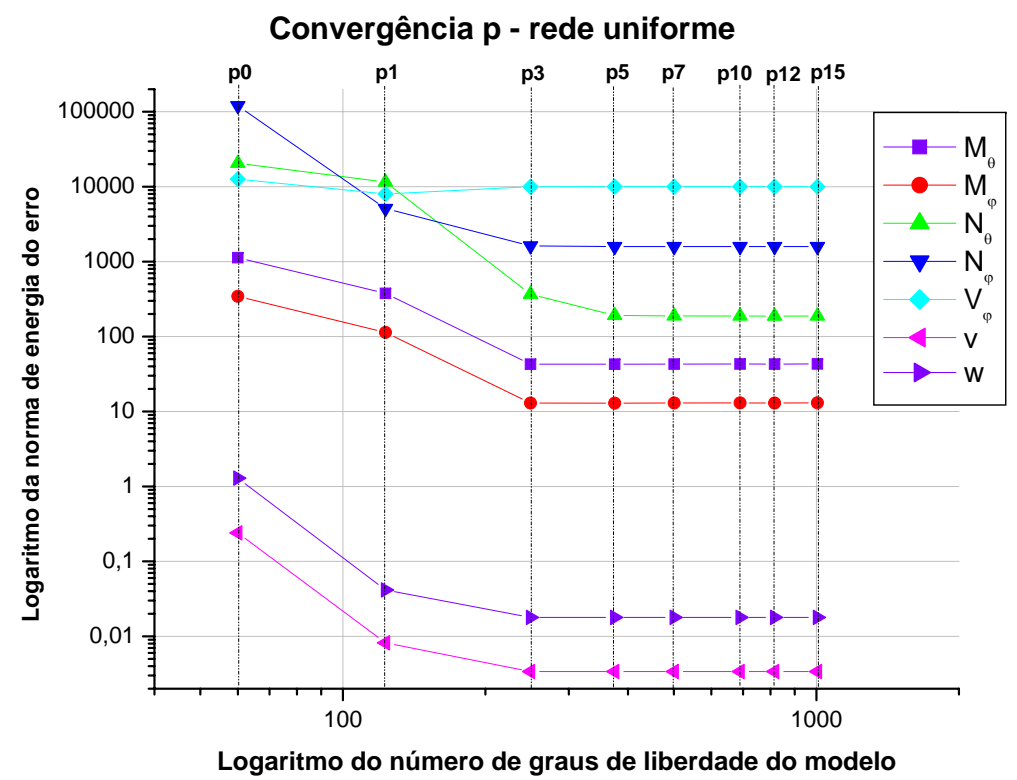

Figura 97: Convergência - refino $p$

plos anteriores. A taxa de convergência inicial é alta com o uso do refino p. Para polinômios de ordem mais elevada, os resultados tendem a se manter praticamente invariáveis.

O fato do refino p apresentar elevadas taxas de convergência nos primeiro graus de enriquecimento não implica em melhoras contínuas da solução do problema, diferentemente do ocorrido no refino h quando se nota uma progressiva melhora nos resultados. $\mathrm{O}$ não avanço da convergência para solução com enriquecimento polinomial de maior ordem evidencia a necessidade de se melhorar a rede de elementos finitos, a fim de se conseguir valores mais próximos da resposta do problema. 


\section{Conclusão}

O Método dos Elementos Finitos Generalizados consiste em ferramenta nãoconvencional eficiente para a análise do comportamento de estruturas em casca, entre outras aplicações.

As formulações convencionais, como as que usam o MEF, podem exigir redes muito refinadas de elementos, particularmente se o objetivo for a descrição mais precisa dos campos de esforços internos, o que implica em elevado custo computacional.

O MEFG permite explorar convenientemente o enriquecimento polinomial, explorado neste trabalho, e outras formas de expansão pois ele pode ser aplicado em forma seletiva, dispensando o recurso do aumento exagerado do número de elementos e com relativo menor custo computacional.

Os comentários anteriores são justificados pelos resultados do capítulo 6 .

Nos diferentes problemas propostos, pôde-se constatar que respostas com grau de precisão bastante aceitável foram obtidas com um pequeno aumento na ordem polinomial das funções de aproximação. Mais especificamente constatou-se que o emprego de uma rede de 20 elementos finitos, com enriquecimento polinomial de ordem 3, foi suficiente para obter soluções com pequena margem de erro para esforços e deslocamentos.

A análise dos gráficos de convergência da solução, em relação a uma solução de referência, mostrou que taxas de convergência muito maiores do que aquelas que seriam obtidas com refino h podem ser obtidas com os primeiros graus de enriquecimento.

Entretanto, elevar sucessivamente a ordem das funções de aproximação tem efeito limitado, devido à forte variação nos esforços que se verifica em regiões como a de vinculação com o meio exterior ou a região de acoplamento entre superfícies distintas.

Mantido o recurso ao enriquecimento polinomial, a combinação com o refino h deve ser a melhor alternativa para ulteriores diminuições do erro de aproximação.

Pelo exposto, é possível perceber que as estratégias possuem vantagens, tanto 
o refino h como o refino p. A associação das duas, buscando uma rede ótima (refino hp) para o enriquecimento, certamente pode levar a resultados mais precisos e com uma taxa de convergência bastante elevada e contínua, ficando esta abordagem como uma das principais sugestões para desenvolvimentos futuros.

A implementação de outros tipos de funções de enriquecimento, não-linearidades e a aplicação do método a outras áreas de estudo, como a Mecânica da Fratura e Dano, por exemplo, são mais algumas sugestões de aplicação do método para trabalhos futuros. 


\section{Referências}

AGUIAR, E.; BARBATO, R. Estudo de cestas protendidas para coberturas: da fase de protensão até o carregamento último. 29 Jornadas Sudamericanas de Ingenieria estructural, p. 13-17, Novembro 2000.

AHMAD, S.; IRONS, B. M.; ZIENKIEWICZ, O. C. Anlalysis of thick and thin shell structure by curved finite element. International jounal of numerical method in engineering, v. 2, p. 419-451, 1970.

ALMEIDA, L. R. de. Parabolóides elípticos monolíticos e pré-moldados sobre base retangular. Dissertação (Mestrado) - Escola de Engenharia de São Carlos - Universidade de São Paulo, São Carlos, SP, 1982.

ALVES, M. M. Emprego do método de resíduos ponderados para a análise de tubos. Dissertação (Mestrado) — Escola de Engenharia de São Carlos - Universidade de São Paulo, São Carlos, SP, 2005.

ANSYS Versão 9.0 Documentation.ANSYS, Inc.

ASSAN, A. E. Método dos Elementos Finitos. Primeiros Passos. 2. ed. Campinas, SP: Editora da Unicamp, 2003. (Coleção Livro-Texto).

BARBATO, R. Introdução ao cálculo de coberturas pênseis: Generalidades. EESC-USP 2000.

BARROS, F. B. Método sem malha e Método dos elementos finitos generalizados em análise não-linear de estruturas. Tese (Doutorado) — Escola de Engenharia de São Carlos - Universidade de São Paulo, São Carlos, SP, 2002.

BARROS, F. B.; PROENÇA, S. P. B.; BARCELLOS, C. S. de. A p-adaptive strategy of nonlinear strutural analysis by the generalized finite element method. Fifth World Congress on Computational mechanics, v. 1, 2002.

BELLUZZI, O. Ciencia de la contruccion. Madrid: Aguilar, 1967.

BILlingtON, D. P. Thin shell concrete structures. 2. ed. New York: McGraw-Hill Book Company, 1982.

DINIS, L. M. Notas de aula sobre placas e cascas. Portugal, http://paginas.fe.up.pt/ ldinis/placasecascastexto.htm 2004.

DUARTE, C.; BABUSKA, I.; ODEN, J. Generalized finite element methods for three dimensional structural mechanics problems. Computers Structures, v. 77, n. 2, p. 215-232, 2000. 
DUARTE, C. A.; ODEN, T. J. Hp clouds a-meshless method to solve bondary value problems. Ticam Report, 1995.

FILHO, A. A. Elementos Finitos: A base da tecnologia CAE. 7. ed. SãoPaulo,SP: Editora Érica, 2002.

GRAVINA, P. B. Teoria e cálculo das cascas - Cascas de Revolução. São Paulo, SP: [s.n.], 1957.

MARTINELLI, D. A. O. Notas de aula: Introdução às estruturas em casca de revolução. 1983.

MARTINELLI, D. A. O.; MONTANARI, I.; SAVASSI, W. Placas elásticas. São Carlos, SP, Outubro 2003.

MELENK, J. M.; BABUSKA, I. The partition of unity finite element method: Basic theory and applications. Computer methods and applied mechanics engieering, v. 139, p. 289-314, 1996.

NÓBREGA, S. H. S. Sobre o desenvolvimento de elementos finitos de casca. Uma classe de formulações mistas. Tese (Doutorado) - Universidade de São Paulo., São Paulo, SP, 1997.

NIRSCHL, G. C. Método dos elementos finitos e técnicas de enriquecimento da aproximação aplicados à análise de tubos cilindricos e cascas esféricas. Dissertação (Mestrado) - Escola de Engenharia de São Carlos - Universidade de São Paulo, São Carlos, SP, 2005.

NIRSCHL, G. C.; GARCIA, O. A.; PROENÇA, S. P. B. Método de elementos finitos não convencionais na análise de placas e cascas com simetria de revolução. Congresso Ibero Latino Americano sobre Métodos Computacionais para Engenharia - CILANCE2005, 2005.

OLIVEIRA, M.; BARBATO, R. Coberturas pneumáticas. Congresso Latino Americano Tecnologia e Gestão na Produção de Edifícios: Soluções para o terceiro milênio, v. 1, p. 183-190, 1998.

OLIVEIRA, M.; BARBATO, R. Estruturas de membrana: estado-da-arte e tendências do desenvolvimento. Simpósio Nacional sobre Tensoestruturas, Maio 2002.

PIMENTA, P. M.; PROENÇA, S. P. B.; FREITAS, J. de. Elementos finitos híbridomistos com enriquecimento nodal. $V$ Congresso de metodos numericos en la ingenieria, v. 1, 2002.

PROENÇA, S. P. B. Sobre modelos matemáticos do comportamento não-linear do concreto: Análise crítica e contribuições. Tese (Doutorado) - Escola de Engenharia de São Carlos - Universidade de São Paulo, São Carlos, SP, 1988.

PROENÇA, S. P. B. Notas de aulas sobre cascas de revolução: teorias de membrana e de flexão. EESC-USP 2005.

PROENÇA, S. P. B. Notas de aula sobre introdução aos métodos numéricos. EESC-USP 2006. 
REDDY, J. N. An Introduction to the Finite Element Method. 2. ed. New York: McGraw-Hill International Editions, 1993. (ENgineering Mechanics Series).

SAVASSI, W. Introdução ao Método dos Elementos Finitos em Análise Linear de Estruturas. São Carlos, SP: Serviço Gráfico da EESC/USP, 2000.

SORIANO, H. L. Método de elementos finitos em análise de estruturas. São Paulo,SP: Edusp, 2003.

STROUBOUlIS, T.; BABUSKA, I.; COPPS, K. The design and analysis of the generalized finite element method. Computer methods in applied mechanics and engineering, v. 181, n. 1-3, p. 43-69, August 1998.

SUAREZ, O. A. G. de. Elementos finitos generalizados na análise estática de placas e cascas. Tese (Doutorado) - Universidade Federal de Santa Catarina, Florianópolis, SC, 2003.

SUAREZ, O. A. G. de; PROENÇA, S. P. B. Linear analysis of axis-symmetric plates and shells by the generalized finite element method. Sumetido ao Latin American Journal of Solids and Structur, 2006.

SUAREZ, O. A. G. de; PROENÇA, S. P. B. de. Método dos elementos finitos generalizados na análise linear de placas e cascas axissimétricas. Relatório apresentado a FAPESP, 2005.

SZABÓ, B.; BABUSKA, I. Finite Element Analysis. New York, USA: Wiley-Interscience Publication, 1991.

TIMOSHENKO, S.; GOODIER, J. Teoria da Elasticidade. 3. ed. Rio de Janeiro, RJ: Guanabara, 1980.

TIMOSHENKO, S.; WOINOWSKY-KRIEGER, S. Theory of Plates and Shells. 2. ed. New York: McGraw-Hill Book Company, 1959.

ZAGOTTIS, D. de. Introdução à Teoria das Placas e das Cascas. São Paulo, SP: [s.n.], 1973. (Pontes e Grandes Estruturas, v. 5).

ZIENKIEWICZ, O.; R.L.TAYLOR. The Finite Element Method. 5. ed. Oxford: Butterworth-Heinemnn, 2000. 
